\title{
A fractional-order multistable locally-active memristor and its chaotic system with transient transition, state jump
}

\section{Xie Wenli}

Hunan University

Chunhua Wang ( $\nabla$ wch1227164@hnu.edu.cn )

Hunan University https://orcid.org/0000-0001-6522-9795

Lin Hairong

Hunan University

\section{Research Article}

Keywords: Locally active memristor, Multistable, Fractional-order, Transient transition, State jump, Antimonotonicity

Posted Date: March 18th, 2021

DOl: https://doi.org/10.21203/rs.3.rs-316323/v1

License: (a) (1) This work is licensed under a Creative Commons Attribution 4.0 International License. Read Full License 


\title{
A fractional-order multistable locally-active memristor and its chaotic system with transient transition, state jump
}

\author{
Wenli Xie • Chunhua Wang • Hairong Lin
}

Received:17 December 2020 / Accepted:

\begin{abstract}
Fractional calculus is closer to reality and has the same memory characteristics as memristor. Therefore, a fractional-order multistable locally active memristor is proposed for the first time in this paper, which has infinitely many coexisting pinched hysteresis loops under different initial states and wide locally active regions. Through the theoretical and numerical analysis, it is found that the fractional-order memristor has stronger locally active and memory characteristics and wider nonvolatile ranges than the integer-order memristor. Furthermore, this fractional-order memristor is applied in a chaotic system. It is found that oscillations occur only within the locally active regions. This chaotic system not only has complex and rich nonlinear dynamics such as infinitely many discrete equilibrium points, multistability, anti-monotonicity but also produces two new phenomena that have not been found in other chaotic systems after neglecting some initial transients. The first one is transient transition: the behavior of transient chaotic and transient period transition alternately occurring. The second is state jump: the behavior of period-4 oscillation or chaotic oscillation jumping to period-2 oscillation. Finally, the circuit simulation of the fractional-order multistable locally active memristive chaotic system using PSIM is carried out to verify the validity of the numerical simulation results.
\end{abstract}

W. Xie. C. Wang (ه) (D. H. Lin (D.

College of Computer Science and Electronic Engineering $\mathrm{Hu}-$ nan University, Changsha 410082, China

W. Xie

E-mail: wlxie123456@163.com

C. Wang

E-mail: wch1227164@hnu.edu.cn

H. Lin

E-mail: haironglin66@126.com
Keywords Locally active memristor - Multistable . Fractional-order · Transient transition · State jump . Anti-monotonicity

\section{Introduction}

Fractional calculus is an extension of integral calculus. Owing to the objects are usually fractional-order, in real life [1], it is more accurate to describe objects using fractional-order than integer-order. In addition, fractional calculus has the memory characteristic with respect to time. Memristor is the fourth fundamental circuit element [2] which is widely used in chaotic neural networks [3-6], circuit design [7-9], secure communications [10-12], bio-simulation circuit [13] and logic circuit [14]. Memristor has the same memory characteristic as fractional calculus, so memristor can be extended to fractional-order. Coopmans et al. proposed the concept of fractional-order memristive systems and investigated the mathematical relationship between the dynamics of memristive systems and the fractional calculus [15]. Petras proposed a fractional-order flux-controlled memristor for the first time and applied it in Chua's circuit. By fractional-order differential, they got a chaotic system in which total order less than three [16]. Cafagna and Grassi proposed the simplest fractional-order memristive chaotic system and proved the existence of chaotic attractors by numerical simulation. They also used 0-1 test to further confirm the existence of chaos [17]. A fractional-order quartic polynomial memristive Chua's circuit can generate a four-scroll attractor and the effect of initial value and fractional-order on dynamics was studied [18]. Si et al. proposed a fractional-order charge-controlled memristor and found triple-loop I-V curves for the first time whose area decreased with the 
decrease of fractional-order [19]. Yang and Xu proposed a fractional-order flux-controlled memristor and gave the computational formulas of areas of pinched hysteresis loops. Besides, the effect of fractional-order on the memristor was studied [20]. Yu and Wang investigated the fractional-order HPTiO-2 memristor model and discussed the voltage-ampere characteristics of the circuit in which the memristor was in series with capacitance or inductance [21]. In Ref. [22], the fractional-order Chua's circuit model with a fractional-order memristor was proposed and the intermittent chaos caused by bifurcation was studied.

The locally active was defined by Chua [23], it is the origin of complexity [24], because it provided an effective method to study complex nonlinear dynamics. Locally active devices are necessary for oscillating systems [25]. Oscillations occur only in locally active regions [26]. Refs. [27, 28] stated that the nonlinear dynamics of locally active memristors are richer with the increase of the number of stable equilibrium points. However, a lot of published locally active memristors have only one to three stable pinched hysteresis loops and limited locally active regions. For example, Chua proposed a corsage memristor with one pinched hysteresis loop and limited locally active ranges [29]. Mannan et al. analyzed Chua's corsage memristor from the bifurcation chart and complex frequency domain. The oscillation was analyzed from the theory of locally active and the edge of chaos [30]. Jin et al. proposed a voltagecontrolled locally active memristors with two pinched hysteresis loops and limited locally active ranges and applied the memristor in a simple circuit which can generate chaotic attractor [31]. Chang et al. proposed a bi-stable locally active memristor with twin symmetrical locally activity ranges and applied it in an oscillator system to analyze the dynamics of memristor [32]. Mannan et al. proposed a 4-lobe Chua's corsage memristor with three pinched hysteresis loops and limited locally active ranges, they analyzed the phenomenon of the edge of chaos [33]. Wang et al. proposed a locally active memristor with two pinched hysteresis loops and four locally active regions. The effect of locally active memristors on the complexity of systems was discussed [34]. Tang and Wang proposed a locally active memristor with two pinched hysteresis loops and wide locally active regions, and found that firing behaviors occur only within the locally active regions [35]. Lin et al. proposed a tri-stable locally active memristor with five locally active regions by which constructed 2D HR neurons could generate multiple firing patterns [36]. Ref. [37] proposed a locally active memristor with three pinched hysteresis loops and wide locally-active regions by introducing polynomial functions into the equation of state.
In previous studies, the locally active memristor has only one to three stable pinched hysteresis loops. Only Ref. [38] proposed a multistable locally active memristor by which constructed neural networks could generate controllable multi-stable behavior.

However, it is worth noting that the fractional-order multistable locally active memristor has not been studied. Motivated by these considerations, a fractionalorder multistable locally active memristor is proposed for the first time in this paper, and it has infinitely many coexisting pinched hysteresis loops and wide locally active regions. The effects of parameters on the nonvolatile and multistable characteristics of the memristor are investigated. Through analysis, we find that the fractional-order memristor has stronger locally active and memory characteristics and wider nonvolatile ranges than the integer-order memristor. Furthermore, this fractional-order multistable locally active memristor is applied in a simple chaotic system. It is found that oscillations occur only within the locally active regions. Through the theoretical and numerical analysis, It is found that the fractional-order system not only has complex dynamics but also presents two phenomena which have not been found in other chaotic systems, these phenomena are called transient transition and state jump.

The rest of this paper is organized as follows: Section 2 introduces the mathematical model of fractionalorder multistable locally active memristor and uses the power-off plot (POP) and DC v-i Loci to verify the nonvolatile, multistable and locally active characteristics of this memristor. In section 3, a fractional-order chaotic system based on this fractional-order multistable locally active memristor is established and the nonlinear dynamics of this system are numerically revealed using bifurcation diagrams, Lyapunov exponent spectrum, and phase portraits. In section 4, the circuit simulation is carried out by PSIM to verify the validity of the numerical simulation results. Section 5 summarizes this paper.

\section{Fractional-order multistable locally active memristor}

2.1 The fractional-order multistable locally active memristor model

Fractional calculus generalizes traditional calculus to non-integer integrals and differentials [39]. In this paper, we consider using Caputo's definition to obtain fractional derivatives [40]. So the fractional-order deriva- 
tive of $\mathrm{f}(\mathrm{t})$ defines:

${ }_{r} D_{t}^{\alpha} f(t)=\frac{1}{\Gamma(n-\alpha)} \int_{r}^{t} \frac{f^{(n)}(\tau)}{(t-\tau)^{\alpha-n+1}} d \tau,(n-1<\alpha \leq n)$

where $\alpha(\alpha \epsilon(0,1])$ is the fundamental operator of fractional calculus or called $\alpha$-order, $\Gamma$ (.) is Gamma function.

Memristor can be regarded as a resistor whose resistance varies with the charge or the magnetic flux. According to the definition of memristor [38], a fractionalorder multistable locally active memristor is proposed as follows:

$\left\{\begin{array}{l}r D_{t}^{\alpha} x=-v+\sin (m x) \\ i=n x v\end{array}\right.$

where $i$ and $v$ are the input current and input voltage, $\mathrm{x}$ is the state variable of the memristor, $\mathrm{nx}$ is the memductance, $\mathrm{m}$ and $\mathrm{n}$ are parameters.

To explore the effect of $\alpha$-order on the characteristics of memristor, we can set parameters $m=1, n=1$ and consider that the memristor is driven by a sinusoidal signal source with amplitude $\mathrm{A}=1 \mathrm{~V}$ and frequency $\mathrm{f}=3.14 \mathrm{~Hz}$ The larger the area of the hysteresis loop, the better the memory characteristics [20]. From the pinched hysteresis loops under different $\alpha$-order shown in Fig. 1, it is found that with the increase of $\alpha$-order, the area of pinched hysteresis loops decreases. So the fractionalorder multistable memristor has stronger memory characteristics than the integer-order multistable memristor [38]. In addition, the memductance can be expressed as the slope of pinched hysteresis loop [31]. From Fig. 1, it is found that the smaller the $\alpha$-order, the greater the negative slope at the origin. Owing to the memristor is active when the memductance is less than 0 , the fractional-order multistable memristor has stronger locally active characteristics than the integer-order multistable memristor [38].

To explore the effect of frequency on the memristor, we can set parameters $\mathrm{m}=1, \mathrm{n}=1$ and $\alpha=0.9$. The memristor is driven by a sinusoidal signal source with amplitude $\mathrm{A}=1 \mathrm{~V}$ at different frequencies. From Fig.2, it is found that with the increase of frequencies, the area of pinched hysteresis loops decreases and gradually approaches a single-valued function.

\subsection{Nonvolatile and multistable}

Nonvolatile allows memristor to retain memory when powered off and it can be proved by POP curve [29]. If the POP curve intersects the $\mathrm{x}$-axis at two or more points with a negative slope, the fractional-order memristor is nonvolatile. When the memristor is powered off,

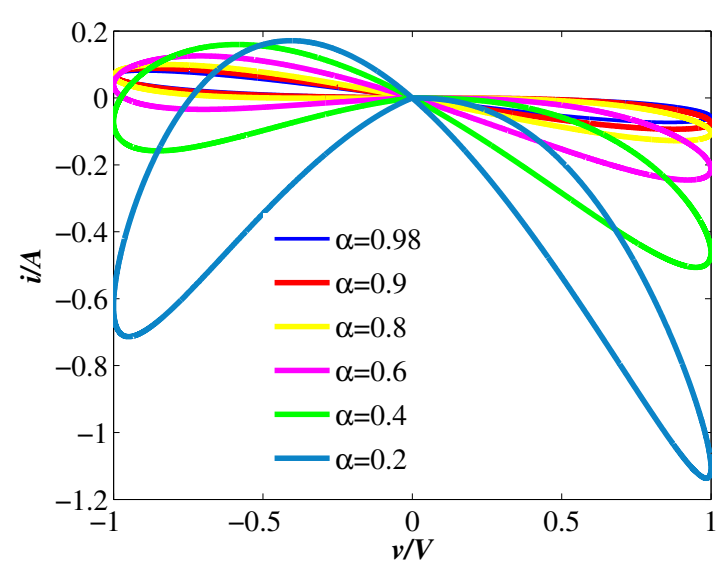

Fig. 1 Pinched hysteresis loop for different $\alpha$-orders, when parameters $m=1$ and $n=1$

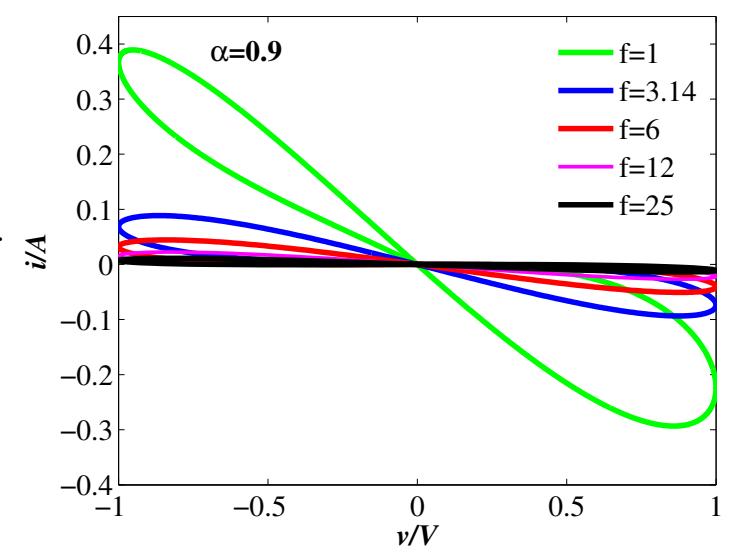

Fig. 2 Pinched hysteresis loop for different frequencies, when parameters $\mathrm{m}=1, \mathrm{n}=1$ and $\alpha=0.9$

the dynamical trajectory of the memristor must tend to the corresponding stable equilibrium point which means the multistability of memristor [38]. When the memristor is powered off, the input voltage $\mathrm{v}=0$. From Eq. (2), the state variable of the memristor becomes:

${ }_{r} D_{t}^{\alpha} x=\sin (m x)$

From Eq. (3), we know ${ }_{r} D_{t}^{\alpha} \mathrm{x}$ intersects the $\mathrm{x}$-axis at these points $x_{e}=k \pi / m$, where $\mathrm{k} \epsilon \mathrm{z}$. And when $\mathrm{k}=2 \mathrm{i}+1$, where $\mathrm{i} \epsilon \mathrm{z}$, the slopes at $x_{e}$ are negative, so $x_{e}$ are stable equilibrium points. And when $\mathrm{k}=2 \mathrm{i}+2, x_{e}$ are unstable equilibrium points. Therefore, the attraction domains of $x_{\text {stable }}=(2 \mathrm{i}+1) \pi / \mathrm{m}$ are $(2 \mathrm{i} \pi / \mathrm{m},(2 \mathrm{i}+2) \pi / \mathrm{m})$. Assuming that the nonvolatile ranges of $x_{\text {stable }}$ are the length of attraction domains, the expression of nonvolatile ranges is described as follows:

$\mathrm{L}_{\text {pop }}=(2 i+2) \pi / m-2 i \pi / m=2 \pi / m$

From Eqs. $(3,4)$, we know parameter m determines the location and the nonvolatile ranges of $x_{\text {stable. The }}$ 


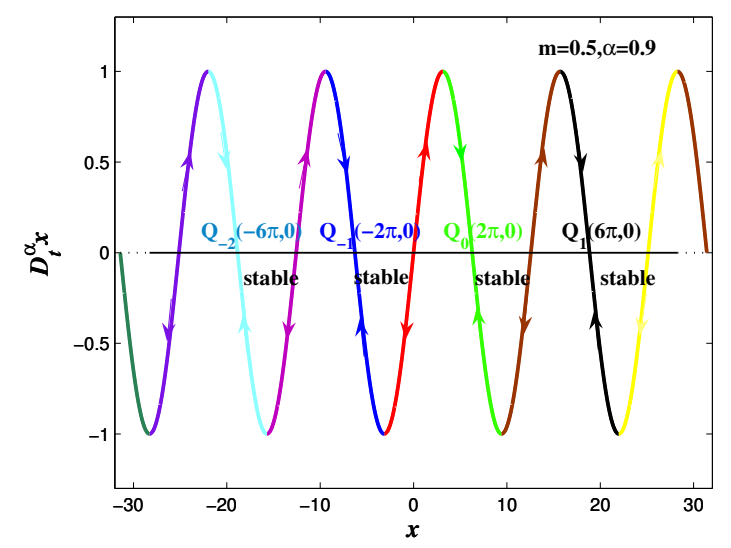

Fig. 3 POP curve of the memristore, when parameters $\mathrm{m}=0.5, \mathrm{n}=1$ and $\alpha=0.9$

smaller the parameter $\mathrm{m}$, the wider the nonvolatile ranges. When parameter $\mathrm{m}<1$, the fractional-order memristor has wider nonvolatile ranges than the integer-order memristor [38].

Setting parameters $\mathrm{m}=0.5, \mathrm{n}=1$ and $\alpha=0.9$, from Eq.(3), we can draw the POP curve shown in Fig. 3. It is found that ${ }_{r} D_{t}^{\alpha} \mathrm{x}$ intersects the $\mathrm{x}$-axis at nine points in which $Q_{-2}(-6 \pi, 0), Q_{-1}(-2 \pi, 0), Q_{0}(2 \pi, 0), Q_{1}(6 \pi$, $0)$ have negative slope, so they are stable, therefore the memristor is nonvolatile. When $v_{m}=\sin (6.28 \pi \mathrm{t})$, Fig. 4 shows the coexisting pinched hysteresis loops under different initial states. It is found that when $x_{0}=-6 \pi$, the slope at the origin of the light black curve tends to $-6 \pi$, which is memductance when the memristor is powered off [32]. When $x_{0}=-2 \pi$, the slope at the origin of the blue curve tends to $-2 \pi$. When $x_{0}=2 \pi$, the slope at the origin of the green curve tends to $2 \pi$. When $x_{0}=6 \pi$, the slope at the origin of the black curve tends to $6 \pi$. The larger the initial value $x_{0}$ ranges, the more coexisting pinched hysteresis, so the fractional-order locally active memristor is multistable.

\subsection{Wide locally active regions}

DC voltage-current analysis can judge whether the memristor is locally active. If the $\mathrm{DC}$ v-i Loci has a negative slope, memristor is locally active [32]. To verify the locally active characteristic of fractional-order memristor, set ${ }_{r} D_{t}^{\alpha} \mathrm{x}=0$, Eq. (2) can be written as follows:

$$
\left\{\begin{array}{l}
v=\sin (m x) \\
i=n x v
\end{array}\right.
$$

Setting $\mathrm{x} \epsilon(-10,8], \mathrm{m}=1$ and $\mathrm{n}=1$. From Eq. (5), we can draw the DC v-i Loci shown in Fig. 5. It is found that the slope of the four curves of dark, purple, green

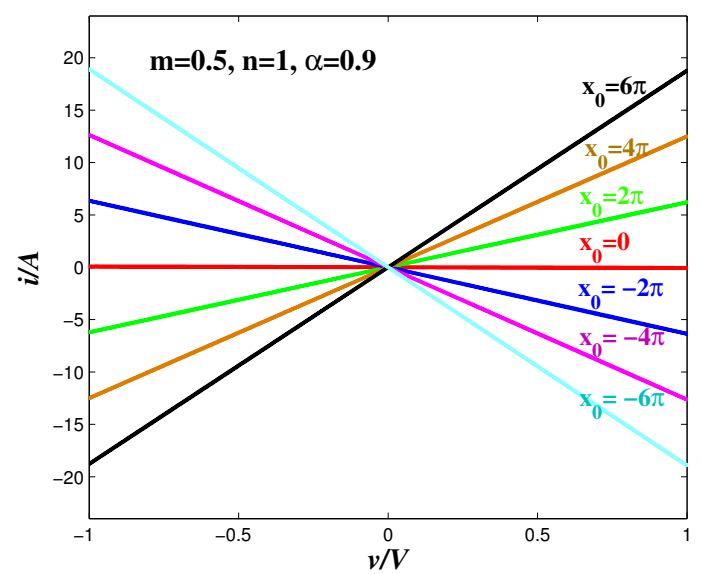

Fig. 4 Coexisting Pinched hysteresis loops, , when parameters $\mathrm{m}=0.5, \mathrm{n}=1$ and $\alpha=0.9$

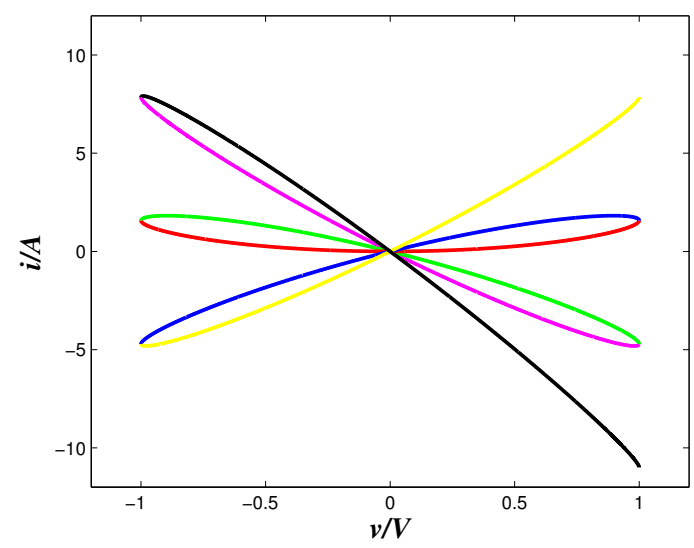

Fig. 5 Locally active ranges when $x_{0} \epsilon(-10,8]$, parameters $\mathrm{m}=0.5$ and $\mathrm{n}=1$

and part of red are negative, so the memristor is locally active. Extending the range of $\mathrm{x}$, we can draw Fig. 6 which shows these purple curves drawn in $x_{0}<0$ have negative slopes. So the whole part of $x_{0}<0$ is active. In conclusion, the fractional-order multistable memristor has wide locally active regions.

Through the above discussion, it is found that the fractional-order multistable locally active memristor not only has infinitely many coexisting pinched hysteresis loops and wide locally active regions but also has stronger locally active and memory characteristics and wider nonvolatile ranges than the integer-order memristor [38]. 


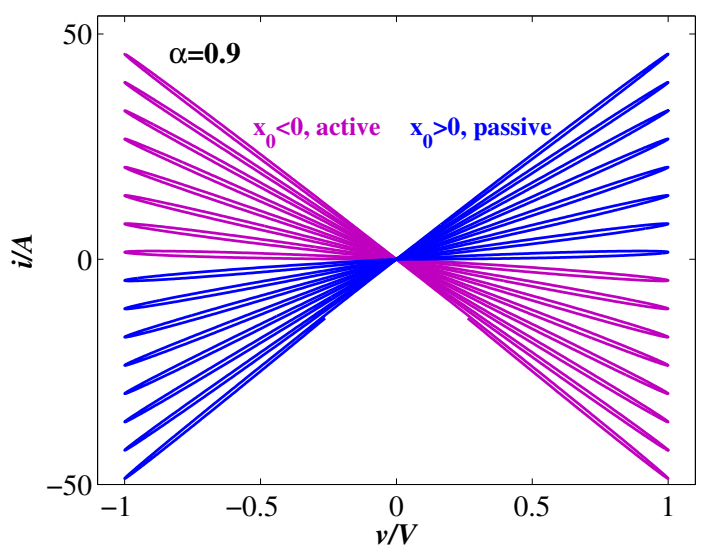

Fig. 6 Locally active ranges when $x_{0} \epsilon[-50,50]$, when parameters $\mathrm{m}=1, \mathrm{n}=1$ and $\alpha=0.9$

\section{Fractional-order multistable locally active memristive chaotic system}

3.1 A 4D fractional-order multistable locally active memristive chaotic system model

From Poincare-Bendixson theory, if an autonomous system generates chaos, its equations satisfy at least three variables and one nonlinear term. J.Kengne et al. proposed a 3D simple autonomous jerk chaotic system with a cubic term which is described as follows [41]:

$\left\{\begin{array}{l}\frac{d x}{d t}=y \\ \frac{d y}{d t}=a z \\ \frac{d z}{d t}=x-b y-z-x^{3}\end{array}\right.$

The fractional-order memristor described in Eq. (2) can be applied in system (6). After doing fractional calculus, a new 4D fractional-order memristive chaotic system is established as follows:

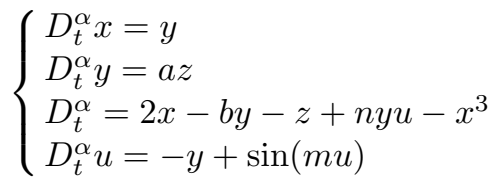

where $u$ denotes the state variable of memristor, $a, b$, $\mathrm{m}$ and $\mathrm{n}$ are the parameters. And $\alpha \epsilon(0,1]$, when $\alpha=1$, system (7) becomes an integer-order chaotic system.

Setting the left-hand-side of Eq. (7) to 0, it is found that whatever parameters $\mathrm{a}, \mathrm{b}$ and $\mathrm{n}$ are, system (7) has three types of equilibrium points which are $(0,0,0$, $\mathrm{k} \pi / \mathrm{m}),(\sqrt{2}, 0,0, \mathrm{k} \pi / \mathrm{m})$ and $(-\sqrt{2}, 0,0, \mathrm{k} \pi / \mathrm{m})$, where $\mathrm{k} \epsilon \mathrm{Z}$.

\subsection{Stability analysis of equilibrium points}

For simplicity, these three type equilibrium points are described as $E_{x_{0}}=\left(x_{0}, 0,0, \mathrm{k} \pi / \mathrm{m}\right)$. When $x_{0}=0$, we can obtain the first type of equilibrium point $E_{x_{0}}=E_{1 k}$ $=(0,0,0, \mathrm{k} \pi / \mathrm{m})$, when $x_{0}=\sqrt{2}$, we can obtain the second type of equilibrium point $E_{x_{0}}=E_{2 k}=(\sqrt{2}, 0,0$, $\mathrm{k} \pi / \mathrm{m})$, when $x_{0}=-\sqrt{2}$, we can obtain the third type of equilibrium point $E_{x_{0}}=E_{3 k}=(-\sqrt{2}, 0,0, \mathrm{k} \pi / \mathrm{m})$.

The Jacobian matrix of system $(7)$ at $E_{x_{0}}$ is obtained as follows:

$$
J_{\Sigma}=\left(\begin{array}{cccc}
0 & 1 & 0 & 0 \\
0 & 0 & a & 0 \\
2-3 x_{0}^{2} & -b+n k \pi / m & -1 & 0 \\
0 & -1 & 0 & m \cos (k \pi)
\end{array}\right)
$$

The characteristic polynomial of the Jacobian matrix (8) is described as follows:

$(m \cos (k \pi)-\lambda)\left[\lambda^{3}+\lambda^{2}+(a b-a n k \pi / m) \lambda+\left(3 a x_{0}^{2}-2 a\right)\right]=0$

Lemma1 [42]: The fractional-order nonlinear system

$D_{t}^{\alpha} X=f(X), 0<\alpha \leq 1$

is asymptotically stable at the equilibrium $\mathrm{E}\left(x_{0}, y_{0}, z_{0}\right.$, $u_{0}$ ), if all eigenvalues $\lambda$ of Jacobian matrix $J_{E}$ satisfy the condition:

$|\arg (\lambda)|>\frac{\alpha \pi}{2}$

where $\mathrm{X}=(x, y, z, u)^{T}, \mathrm{f}(\mathrm{X})=\left[f_{1}(x), f_{2}(x), f_{3}(x), f_{4}(x)\right]^{T}$ $f_{i}(X)=f_{i}(x, y, z, u)(i=1,2,3,4)$, and $\arg (\lambda)$ is the principal argument of eigenvalue $\lambda$.

Whatever parameters a, b and n are, Eq. (9) have a fixed solution $\lambda_{1}=\cos (\mathrm{k} \pi)= \pm 1 / \mathrm{m}$. Take roots $\lambda_{1}$ out of Eq.(9), the rest part of characteristic polynomial can be described as follows:

$\lambda^{3}+\lambda^{2}+(a b-a n k \pi / m) \lambda+\left(3 a x_{0}^{2}-2 a\right)=0$

Assuming that the roots of Eq. (12) are $\lambda_{2}, \lambda_{3}$ and $\lambda_{4}$. To obtain the stability of equilibrium points, the characteristic roots of Eq. (12) are discussed as follows:

(i)According to the criterion of Routh-Hurwitz, $\lambda_{2}$, $\lambda_{3}$ and $\lambda_{4}$ all have negative real parts, when the following inequalities are true.

$\mathrm{a}_{1}>0, \mathrm{a}_{1} \mathrm{a}_{2}-\mathrm{a}_{3}>0, \mathrm{a}_{3}\left(\mathrm{a}_{1} \mathrm{a}_{2}-\mathrm{a}_{3}\right)>0$

where $a_{1}, a_{2}, a_{3}$ denote the coefficients of $\lambda_{2}, \lambda$ and constant term in Eq. (12), so we can obtain $a_{1}=1, a_{2}=$ ab$\operatorname{ank} \pi / \mathrm{m}$ and $a_{3}=3 \mathrm{a} x_{0}^{2}-2 \mathrm{a}$, and expression (13) can be simplified as follows:

$\left\{\begin{array}{l}b-n k \pi / m+2-3 x_{0}^{2}>0 \\ \left(3 x_{0}^{2}-2\right)\left(b-n k \pi / m+2-3 x_{0}^{2}\right)>0\end{array}\right.$

If expression (14) is true, $\lambda_{2}, \lambda_{3}$ and $\lambda_{4}$ all have negative real parts. 
Table 1 The eigenvalues and stabilities of equilibrium points

\begin{tabular}{llllllll}
\hline Number & $\mathrm{b}$ & $\lambda_{2}\left(E_{1 k}\right)$ & $\lambda_{3,4}\left(E_{1 k}\right)$ & $\begin{array}{l}\text { Stability } \\
\text { of equilibrium }\end{array}$ & $\lambda_{2}\left(E_{2 k, 3 k}\right)$ & $\lambda_{3,4}\left(E_{2 k, 3 k}\right)$ & $\begin{array}{l}\text { Stability } \\
\text { of equilibrium }\end{array}$ \\
\hline $\mathrm{I}$ & $\mathrm{b}>\mathrm{nk} \pi / \mathrm{m}+4$ & $>0$ & $-\mathrm{p} \pm \mathrm{qi}$ & Unstable & $-\mathrm{p} \pm \mathrm{qi}$ & $-\mathrm{p} \pm \mathrm{qi}$ & Stable \\
$\mathrm{II}$ & $\mathrm{b}<\mathrm{nk} \pi / \mathrm{m}+4$ & $>0$ & $-\mathrm{p} \pm \mathrm{qi}$ & Unstable & $<0$ & $\mathrm{p} \pm \mathrm{qi}$ & $\alpha$-related \\
\hline
\end{tabular}

Table 2 The numerical simulation results of equilibrium point stability

\begin{tabular}{|c|c|c|c|c|c|c|}
\hline $\mathrm{k}$ & $\lambda_{2}\left(E_{1 k}\right)$ & $\lambda_{3,4}\left(E_{1 k}\right)$ & Stability of equilibrium & $\lambda_{2}\left(E_{2 k, 3 k}\right)$ & $\lambda_{3,4}\left(E_{2 k, 3 k}\right)$ & Stability of equilibrium \\
\hline $\mathrm{k}=-1$ & 1 & $-1 \pm 2.449 \mathrm{i}$ & Unstable & -2 & $0.5 \pm 2.597 \mathrm{i}$ & $\begin{array}{l}\text { When } \alpha<0.878 \text {, stable. } \\
\alpha>0.878 \text {, saddle-focus } \\
\text { points of index } 2\end{array}$ \\
\hline $\mathrm{k}=-5$ & 0.482 & $-0.741 \pm 3.736 \mathrm{i}$ & Unstable & -1.013 & $0.689 \pm 3.716 \mathrm{i}$ & $\begin{array}{l}\text { When } \alpha<0.83, \text { stable. } \\
\alpha>0.83, \text { saddle-focus } \\
\text { points of index } 2\end{array}$ \\
\hline $\mathrm{k}=-6$ & 0.421 & $-0.711 \pm 4.011 \mathrm{i}$ & Unstable & -0.881 & $-0.594 \pm 3.985 \mathrm{i}$ & Stable \\
\hline $\mathrm{k}=-7$ & 0.374 & $-0.687 \pm 4.270 \mathrm{i}$ & Unstable & -0.776 & $-0.111 \pm 4.243 \mathrm{i}$ & Stable \\
\hline
\end{tabular}

(ii) When $E_{x_{0}}=E_{1 k}=(0,0,0, \mathrm{k} \pi / \mathrm{m})$, substituting $x_{0}=0$ into expression (14), expression (15) is obtained.

$\left\{\begin{array}{l}b-n k \pi / m+2>0 \\ -2(b-n k \pi / m+2)>0\end{array}\right.$

If expression (15) is true, $\lambda_{2}, \lambda_{3}$ and $\lambda_{4}$ all have negative real parts, and $E_{1 k}$ is stable. However, the first inequality of expression (15) is contradictory to the second inequality, so whatever $\mathrm{a}, \mathrm{b}, \mathrm{m}$ and $\mathrm{n}$ are, $\lambda_{2}, \lambda_{3}$ and $\lambda_{4}$ have at least one positive real part. According to the Vieta theorem of cubic equation, we know $\lambda_{2} \lambda_{3} \lambda_{4}=-a_{3}=2 \mathrm{a}>0, \lambda_{2}+\lambda_{3}+\lambda_{4}=-a_{1}=-1<0$, so the roots of Eq.(12) at $E_{1 k}$ are a positive real root and a pair of conjugated complex roots whose real parts are negative, so $E_{1 k}$ is the saddle-focus point of index 1 .

(iii) When $E_{x_{0}}=E_{2 k, 3 k}=( \pm \sqrt{2}, 0,0, \mathrm{k} \pi / \mathrm{m})$, substituting $x_{0}= \pm \sqrt{2}$ into expression (14), expression (16) is obtained.

$\left\{\begin{array}{l}b-n k \pi / m-4>0 \\ 4(b-n k \pi-4)>0\end{array}\right.$

When $\mathrm{b}>\mathrm{nk} \pi / \mathrm{m}+4$, expression (16) is true, so whatever a is, $\lambda_{2}, \lambda_{3}$ and $\lambda_{4}$ all have negative real part, and $E_{2 k, 3 k}$ are stable equilibrium points. When $\mathrm{b}<\mathrm{nk} \pi / \mathrm{m}+4$ expression (16) is not true, so whatever a is, $\lambda_{2}, \lambda_{3}$ and $\lambda_{4}$ have at least one positive real part. According to the Vieta theorem of cubic equation, we know $\lambda_{2} \lambda_{3} \lambda_{4}=-$ $a_{3}=-4 \mathrm{a}<0, \lambda_{2}+\lambda_{3}+\lambda_{4}=-a_{1}=-1<0$, so the roots of Eq. (12) at $E_{2 k, 3 k}$ are a negative real root and a pair of conjugated complex roots whose real parts are positive. By Lemma 1, the stability of $E_{2 k, 3 k}$ is related to $\alpha$-order. When $|\arg (\lambda)|>\alpha \pi / 2$, we can know $E_{2 k, 3 k}$ are asymptotically stable, otherwise, unstable. The above discussion can be concluded in Table 1 .

\subsection{Numerical illustrations}

To better prove the correctness of the above discussion, we make numerical illustrations. Setting parameters $\mathrm{a}=3.5, \mathrm{~b}=0.8, \mathrm{~m}=1$ and $\mathrm{n}=0.2$. From expression (16), we can divide $\mathrm{k}$ into two cases. Case 1: when $0.2 \mathrm{k} \pi+4<0.8$, we can obtain $\mathrm{k}<-5.093$, expression (16) is true. This case corresponds to Number I of Table 1. Case 2: When $0.2 \mathrm{k} \pi+4>0.8$, we can obtain $\mathrm{k}>-5.093$, expression (16) is not true. This case corresponds to Number II of Table 1. The roots of Eq. (12) are shown in Table 2 which are consistent with the results in Table 1 , so the above discussion is correct. When $\mathrm{k}=-5$, 1 , the stability of $E_{2 k, 3 k}$ is related to $\alpha$. By Lemma 1, the results are proved as follows:

When $\left|\frac{\operatorname{Im}\left(\lambda_{3,4}\right)}{\operatorname{Re}\left(\lambda_{3,4}\right)}\right|>\tan \left(\frac{\alpha \pi}{2}\right), E_{2 k, 3 k}$ are asymptotically stable. When $\left|\frac{\operatorname{Im}\left(\lambda_{3,4}\right)}{\operatorname{Re}\left(\lambda_{3,4}\right)}\right|=\tan \left(\frac{\alpha \pi}{2}\right)$, Hopf bifurcation is generated [43]. When $\left|\frac{\operatorname{Im}\left(\lambda_{3,4}\right)}{\operatorname{Re}\left(\lambda_{3,4}\right)}\right|<\tan \left(\frac{\alpha \pi}{2}\right), E_{2 k, 3 k}$ are unstable. The above discussion can be concluded in Table 2 .

\subsection{Complex nonlinear dynamic analysis}

Bifurcation analysis, phase diagrams, and Lyapunov exponents are used to explore the nonlinear dynamics of system (7).

\subsubsection{Oscillation only in locally active regions}

Based on Sec. 2.3, when the initial states of the memristor less than 0 , the fractional-order multistable memristor is locally active. In Eq. (7), u denotes the state variable of the memristor, $u_{0}$ denotes the initial states of the memristor. The bifurcation diagram about $u_{0}$ is 


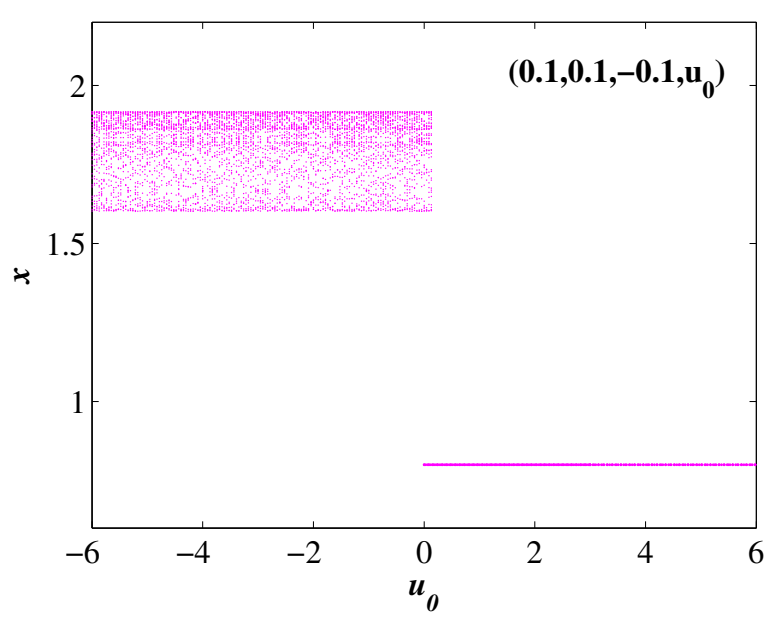

(a)

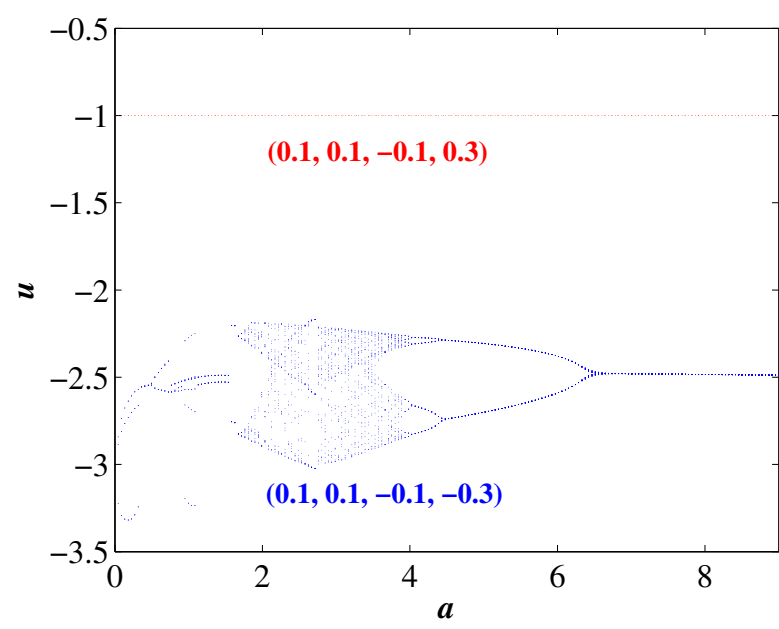

(b)

Fig. 7 (a) Bifurcation diagram for $u_{0}$. (b) Bifurcation diagram for parameter a with initial value $(0.1,0.1,-0.1,-0.3)$ (blue curve) and (0.1, 0.1, -0.1, 0.3) (red curve)

plotted in Fig. $7(\mathrm{a})$ with the fixed parameters $\mathrm{a}=3.5$, $\mathrm{b}=0.8, \mathrm{n}=0.2, \mathrm{~m}=1$ and $\alpha=0.96$. System (7) exhibits completely different oscillation behaviors on either side of $u_{0}=0$. When $u_{0}<0$, the memristor is active and the system is chaotic, when $u_{0}>0$, the memristor is negative and the system cannot oscillate.

The bifurcation diagram about parameter a is plotted in Fig. 7(b) with the fixed parameters $\mathrm{b}=0.8, \mathrm{n}=0.2$, $\mathrm{m}=1$ and $\alpha=0.96$. The blue curve corresponds to the initial value $(0.1,0.1,-0.1,-0.3)$, where system $(7)$ exhibits complex oscillation behaviors. The red curve corresponds to the initial value $(0.1,0.1,-0.1,0.3)$ where system (7) exhibits static behavior. These simulation results suggest that oscillations only occur in the locally active regions.

\subsubsection{Asymptotically periodic solution}

Theorem 1 [44]: The autonomous fractional-order differential equations

$\mathrm{D}^{\alpha} \mathrm{x}_{\mathrm{i}}(\mathrm{t})=f_{\mathrm{i}}\left(\mathrm{x}_{1}(\mathrm{t}), \mathrm{x}_{2}(\mathrm{t}), \ldots \mathrm{x}_{\mathrm{n}}(\mathrm{t})\right), \mathrm{i}=1,2, \ldots, \mathrm{n}$

with $0<\alpha<1$, and $f_{i}$ being some nonlinear function, which cannot have any non-constant exact smooth periodic solution. The same is true for non-autonomous fractional systems [45].

However, the fractional-order system has stable periodic solutions when the lower limit of the Caputo derivative changes to $-\infty$, see Appendix $C$ for the proof process [46]. If the system variables are impulsed periodically, the fractional-order system has periodic trajectories [47]. Besides, it is found that the fractional Brusselator model has limited cycles [48].
Strictly speaking, factional-order systems have not exact periodic solution, that is global period, but have local period, which means that the solution can be periodic over a certain period of time. From the numerical simulation results, the fraction-order system in Ref. [47] sometimes has stable cycle, which isn't an exact periodic solution, and it was called numerical period [46, 47]. Because the exact periodic solution must satisfy the following condition: For any time $t$, in particular, the initial time $t_{0}$, the solution $\mathrm{x}(\mathrm{t})=\mathrm{x}(\mathrm{t}+\mathrm{T})$, and the constant $\mathrm{T}$ is the period of the solution. And the numerical period is only approximations of exact periodic oscillations, always including some aperiodic parts. The periodic solution of the Caputo fractional-order system over a long time is discussed in Ref. [49], it was found that if the observation time is set to a far time, the periodic solution can be obtained, even though it is not periodic over the whole time, the solution after a certain long time is periodic. As a result, there is no global period, but there can be local period.

According to the definition of asymptotically periodic solution [46]: The fractional-order system have asymptotically T-periodic solutions for which $\lim _{t \rightarrow+\infty} \mathrm{x}(\mathrm{t}+\mathrm{T})$ $\mathrm{x}(\mathrm{t})=0$ for a certain $\mathrm{T}>0$. It is found that although the system (7) has not exact periodic solution, but it has the asymptotically periodic solution. The proof process is shown as follows:

For the system (7), the predictor-corrector AdamsBashforth-Moulton in Ref. [50] is used to solve the fractional order differential equation. Set parameters $a=3.5$, $\mathrm{b}=0.8, \mathrm{n}=0.2, \mathrm{~m}=1, \alpha$ is $0.9,0.95$ respectively, initial value $(0.1,0.1,-0.1,0)$ and the step length of simula- 


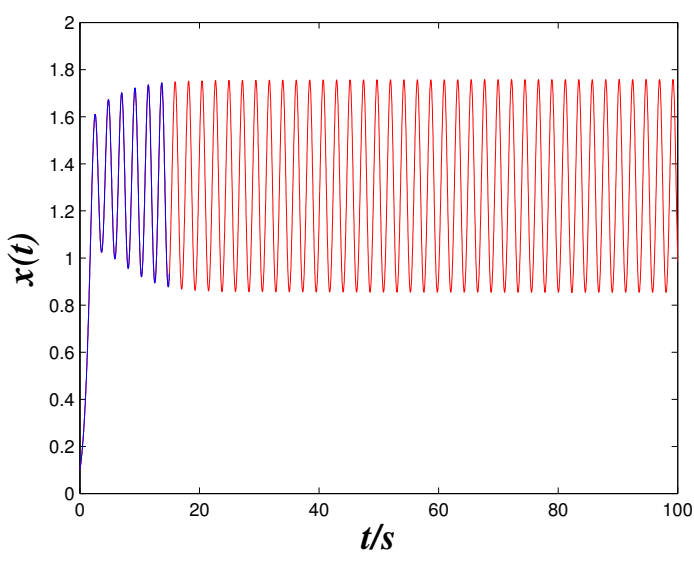

(a)

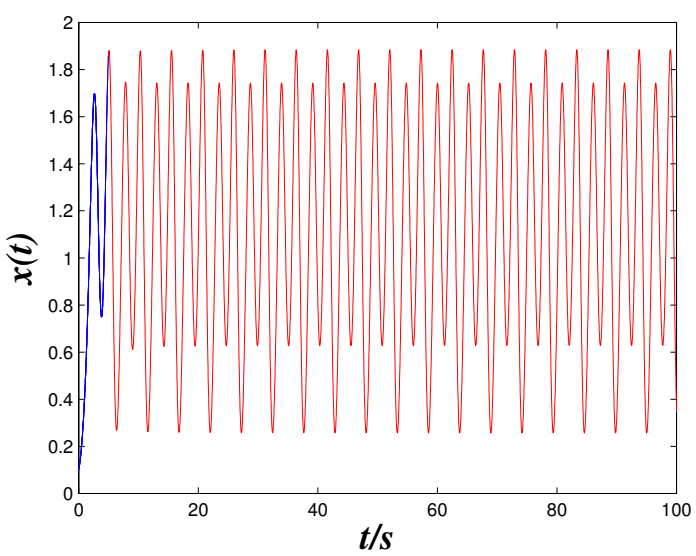

(c)

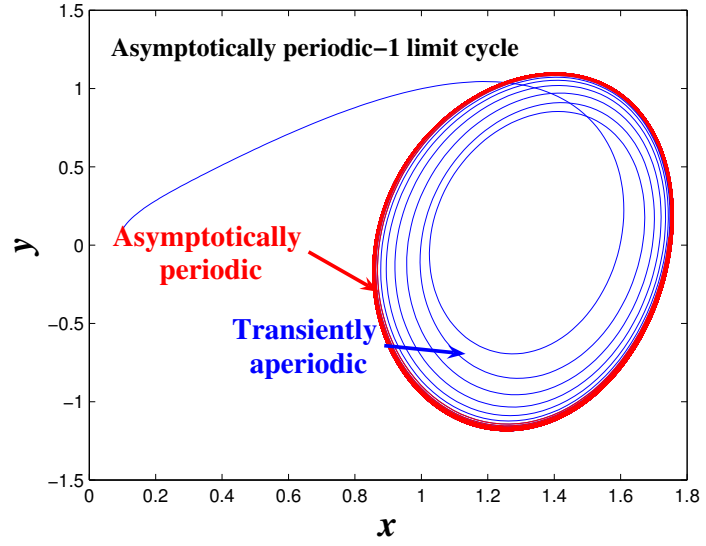

(b)

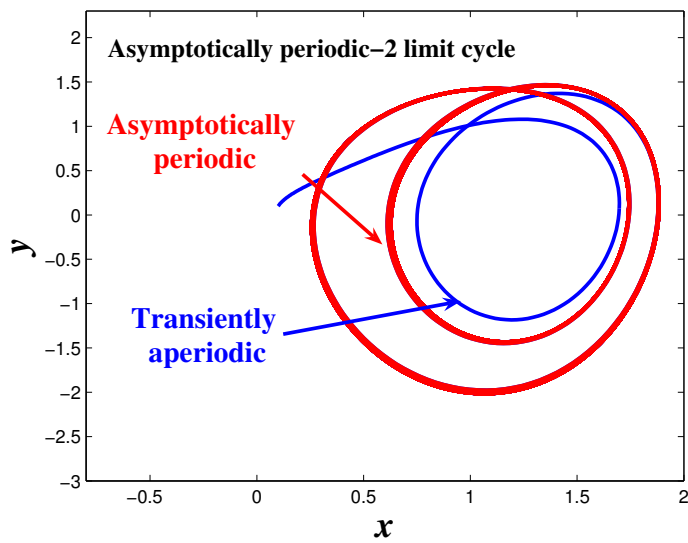

(d)

Fig. 8 (a) the timing diagrams of the system (7) when $\alpha$ is 0.9 . (b) the phase diagrams of system (7) when $\alpha$ is 0.9 . (c) the timing diagrams of the system (7) when $\alpha$ is 0.95 . (d) the phase diagrams of system (7) when $\alpha$ is 0.95 .

tion is 0.02 , Fig. 8 can be obtained. Fig. $8(\mathrm{a})$, (c) are the timing diagrams of the system (7) when $\alpha$ is 0.9 , 0.95 respectively, which represent the solution of system (7) when $t \in(0,100 \mathrm{~s})$. Fig. $8(\mathrm{~b}),(\mathrm{d})$ are the phase diagrams of system (7). From these figures, we can see system (7) is always partially aperiodic (as shown in the blue curve), that is, system (7) has transient aperiodic solution. However, after a relatively short aperiodic period, system (7) has "stable periodic solution" (as shown in the red curve). For Fig. 8(a), we know that $\lim _{t \rightarrow t_{n}} \mathrm{x}(\mathrm{t}+\mathrm{T})-\mathrm{x}(\mathrm{t})=0$, and for Fig. $8(\mathrm{c})$, it is obtained that $\lim _{t \rightarrow t_{m}} \mathrm{x}(\mathrm{t}+2 \mathrm{~T})-\mathrm{x}(\mathrm{t})=0$, where $t_{n}$ and $t_{m}$ are the end time of transient aperiodic solution.

In conclusion, system (7) has not exact periodic solution, but it has the asymptotically periodic solution (Fig. 8(b) shows the asymptotically periodic-1 limit cycle, Fig. 8(d) shows the asymptotically periodic-2 limit cycle). For the convenience of writing, the word "Asymptotically periodic" is simplified as "A-period".

\subsubsection{Lyapunov exponents and bifurcation analysis}

Lyapunov exponents are used to exploring whether a system is in a chaotic state and bifurcation analysis can study the characteristics of a nonlinear system [35]. The method of Ref. [51] is used to solve the Lyapunov exponents. Setting parameters $\mathrm{a}=3.5, \mathrm{~b}=0.8, \mathrm{n}=0.2$ and $\mathrm{m}=1$. Fig. 9 shows the bifurcation diagram and the Lyapunov exponent spectrum for $\alpha$-order. From the blue curve shown in Fig.9 (a), it is found that when $\alpha \in(0,0.878)$, system (7) is stable. When $\alpha=0.878$, system (7) experiences Hopf bifurcation. When $\alpha \in(0.878$, $0.943)$, A-period-1 bifurcation occurs. When $\alpha \in(0.943$, $0.954)$, A-period-2 bifurcation occurs. When $\alpha \in(0.954$, 1), chaos occurs. 


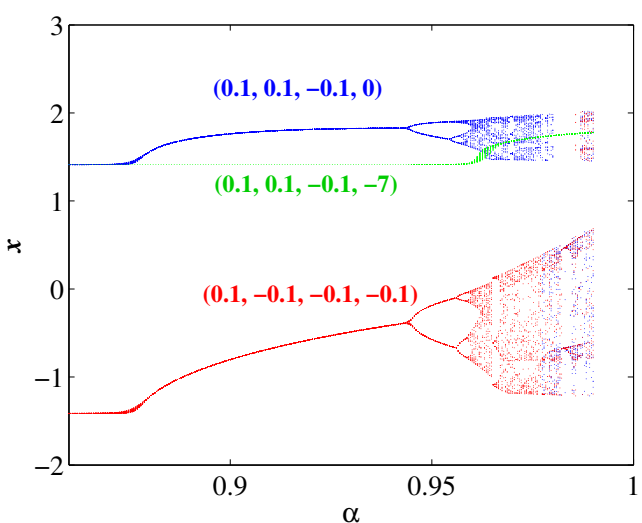

(a)

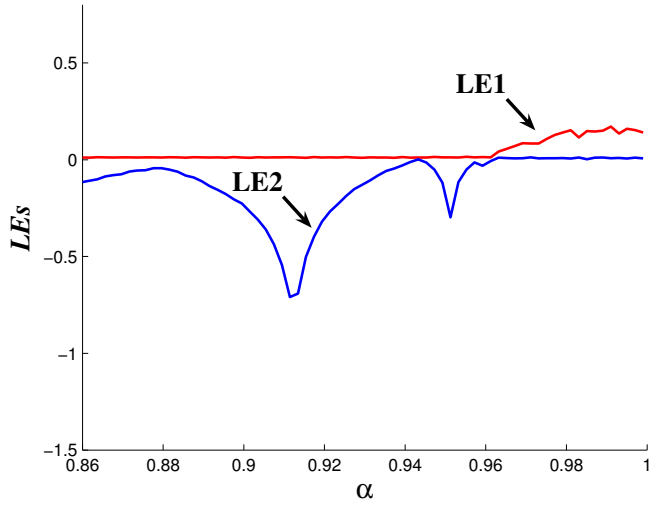

(b)

Fig. 9 (a) Bifurcation diagram for $\alpha$ with initial values $((0.1,0.1,-0.1,0)$ (blue curve), $(0.1,-0.1,-0.1,-0.1)$ (red curve) and $(0.1,0.1,-0.1,-7)$ (green curve). (b) Lyapunov exponent spectrum for initial values $(0.1,0.1,-0.1,0)$.

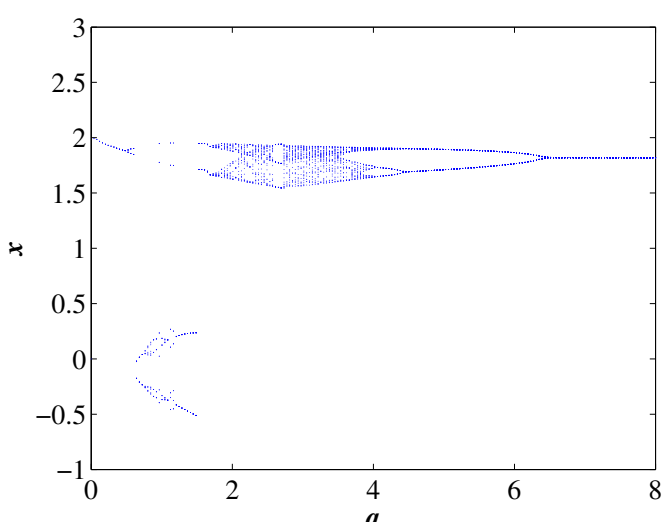

(a)

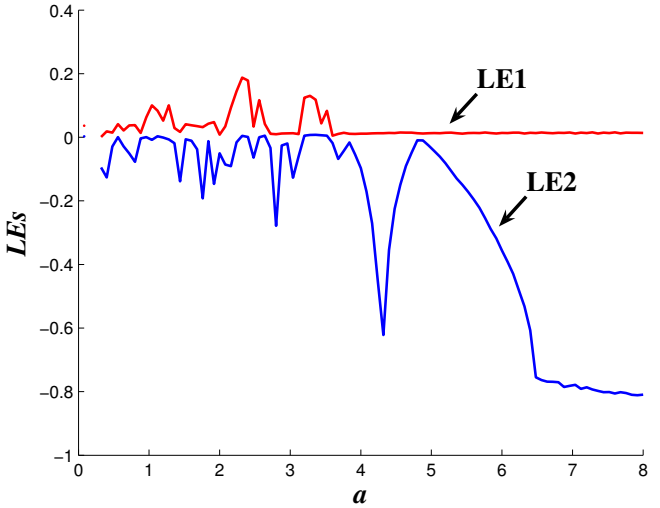

(b)

Fig. 10 (a) Bifurcation diagram for parameter a with initial values $(0.1,0.1,-0.1,0)$. (b) Lyapunov exponent spectrum for parameter a with initial values $(0.1,0.1,-0.1,0)$

Similarly, setting parameters $\alpha=0.96, \mathrm{~b}=0.8, \mathrm{n}=0.2$ and $m=1$. Fig. 10 shows the bifurcation diagram and the Lyapunov exponent spectrum for parameter a. From Fig. 10, it is found that when parameter $\mathrm{a} \in(0.5,1.5)$, A-period-1 bifurcation occurs. When parameter $a \in(1.5$, 1.8), A-period-2 bifurcation occurs. When parameter $\mathrm{a} \in(1.8,4)$, chaos occurs. When parameter $\mathrm{a} \in(4,6.3), \mathrm{A}-$ period-2 bifurcation occurs. And when parameter a $>6.3$, A-period-1 bifurcation occurs.

\subsubsection{Multistability analysis}

If a nonlinear system displays three or more coexisting attractors under different initial values, the system is multistable $[52,53]$. Multistability reflects the sensitivity of the system to initial value. Setting parameters $\mathrm{a}=3.5, \mathrm{~b}=0.8, \mathrm{n}=0.2$ and $\mathrm{m}=1$, after neglecting some initial transients, we can draw Fig. 11. Fig. 11(a) shows
Table 3 The coexisting attractors under different $\alpha$-order

\begin{tabular}{|c|c|}
\hline$\alpha$-order & Steady states \\
\hline $\mathrm{k}=(0,0.875)$ & Three stable points \\
\hline $\mathrm{k}=(0.875,0.88)$ & $\begin{array}{l}\text { Two stable points and } \\
\text { A-period-1 cycle }\end{array}$ \\
\hline $\mathrm{k}=(0.88,0.943)$ & $\begin{array}{l}\text { Two A-period-1 cycles and } \\
\text { one stable point }\end{array}$ \\
\hline $\mathrm{k}=(0.943,0.945)$ & $\begin{array}{l}\text { One A-period-1 cycle, } \\
\text { A-period- } 2 \text { cycle } \\
\text { and one stable point }\end{array}$ \\
\hline $\mathrm{k}=(0.945,0.955)$ & $\begin{array}{l}\text { Two A-period- } 2 \text { cycles } \\
\text { and one stable point } \\
\text { Chaotic attractor, }\end{array}$ \\
\hline $\mathrm{k}=(0.955,0.96)$ & $\begin{array}{l}\text { A-period- } 2 \text { cycle } \\
\text { and one stable point }\end{array}$ \\
\hline $\mathrm{k}=(0.96,1)$ & $\begin{array}{l}\text { Two chaotic attractors } \\
\text { and A-period- } 1 \text { cycle }\end{array}$ \\
\hline
\end{tabular}




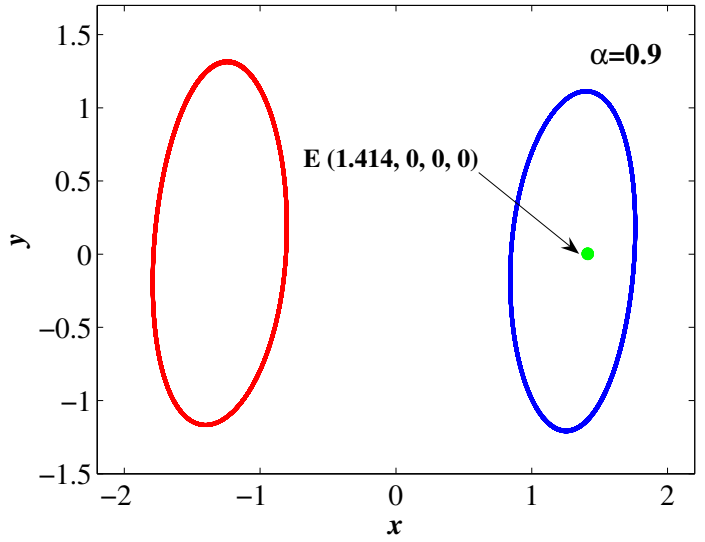

(a)

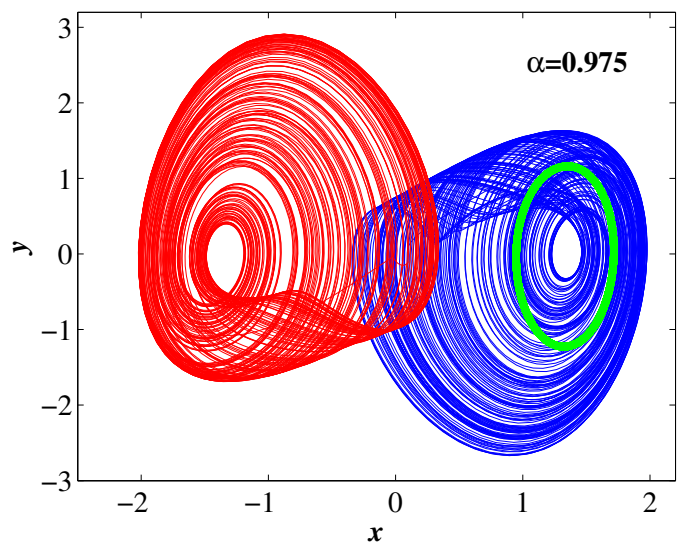

(c)

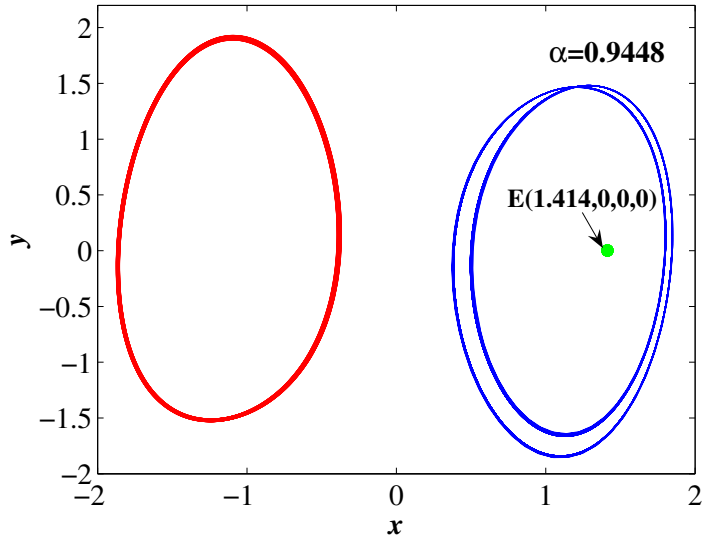

(b)

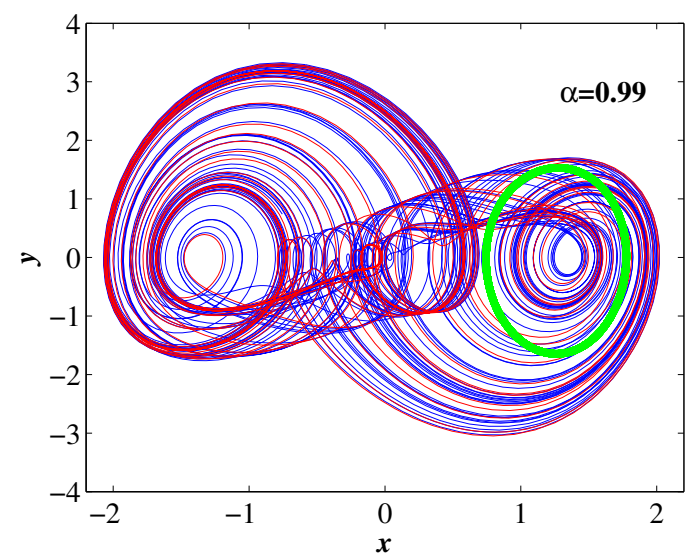

(d)

Fig. 11 Phase portraits for different $\alpha$ with initial values $((0.1,0.1,-0.1,0)$ (blue curve), $((0.1,-0.1,-0.1,-0.1)$ (red curve) and $((0.1,0.1,-0.1,-7)$ (green curve)) (a) $\alpha=0.9$ (b) $\alpha=0.9448$ (c) $\alpha=0.975$ (d) $\alpha=0.99$

the coexistence of two A-period-1 cycles and one stable point in the system when $\alpha=0.9$. Fig. 11(b) shows the coexistence of one A-period-1 cycle and A-period-2 cycle and one stable point in the system when $\alpha=0.9448$. Fig. 11(c) shows the coexistence of two single scrolls and A-period- 1 cycle in the system when $\alpha=0.975$. Fig. $11(\mathrm{~d})$ shows the coexistence of two double-scrolls and A-period-1 cycle in the system when $\alpha=0.99$. From Fig.9(a), we also can see the multistability of system (7) . See Table 3 for details.

\subsubsection{Transient transition}

Because of the appearance of a non-attractive saddle point in the phase space, in earlier times, the orbit behaves as chaos, and then non-chaos which is the definition of transient chaos, on the contrary, the definition of transient period [56]. These two transient behaviors are often found in many chaotic systems [56-58].
However, setting parameters $\mathrm{a}=3.5, \mathrm{~b}=0.8, \mathrm{n}=0.2$, $\mathrm{m}=1, \alpha=0.987$, initial value $(0.1,0.1,-0.1,0)$, the step length of simulation is 0.05 , and the simulation time is $(0,15000 \mathrm{~s})$ (these conditions must be strictly met). system (7) can appear the phenomenon of transient chaos and transient period transition alternately occurring. This phenomenon has not been found in any other integer-order or fractional-order chaotic system. From Fig. 12, it is found that the fractional-order system has not exact periodic solutions(global period), but has transient periodic solutions which are local periodic.

From Fig.12, system (7) produces periodic oscillation, when $t \in(50 \mathrm{~s}, 2000 \mathrm{~s})$. The periodic oscillation transfers to chaotic oscillation, when $t \in(2000 \mathrm{~s}, 2828 \mathrm{~s})$. The chaotic oscillation transfers to periodic oscillation, when $t \in(2828 \mathrm{~s}, 2980 \mathrm{~s})$. After that, chaotic and periodic oscillation transition alternately appears. Fig. 13 shows the transient chaos, when $t \in(1100 \mathrm{~s}, 1400 \mathrm{~s})$, $\operatorname{system}(7)$ generates double-scroll. Fig. 14 shows the transient pe- 


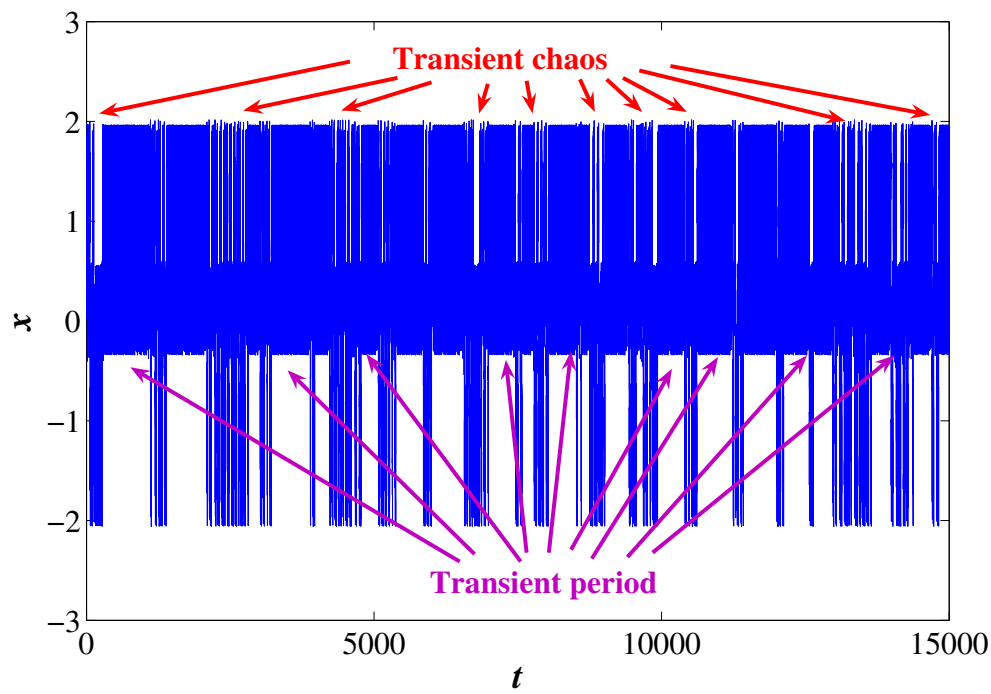

Fig. 12 Time-domain plot of $\mathrm{t} \epsilon(0,15000 \mathrm{~s})$, when $\mathrm{a}=3.5, \mathrm{~b}=0.8, \mathrm{n}=0.2, \mathrm{~m}=1, \alpha=0.987$, initial value $(0.1,0.1,-0.1,0)$

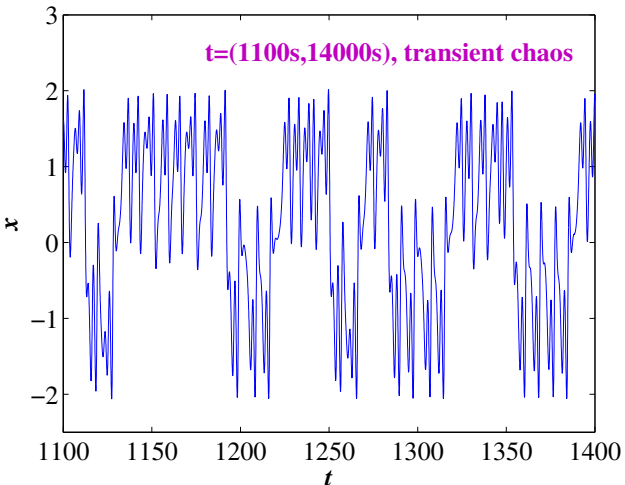

(a)

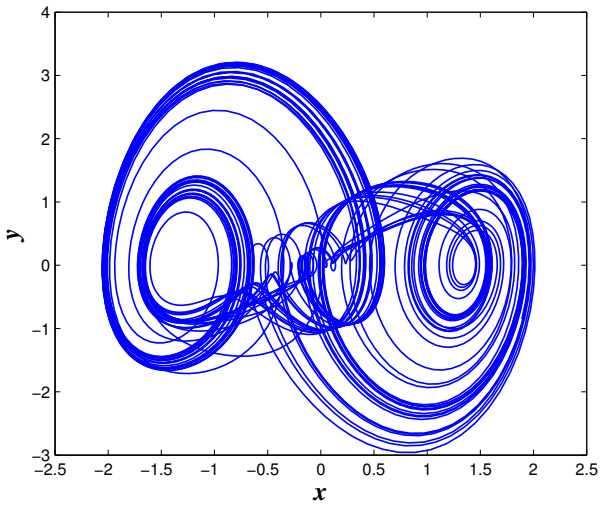

(b)

Fig. 13 (a) Time-domain plot of $t \epsilon(1100 s, 1400 s)$. (b) Phase portraits of $x-y$ axis $t \epsilon(1100 s, 1400 s)$.

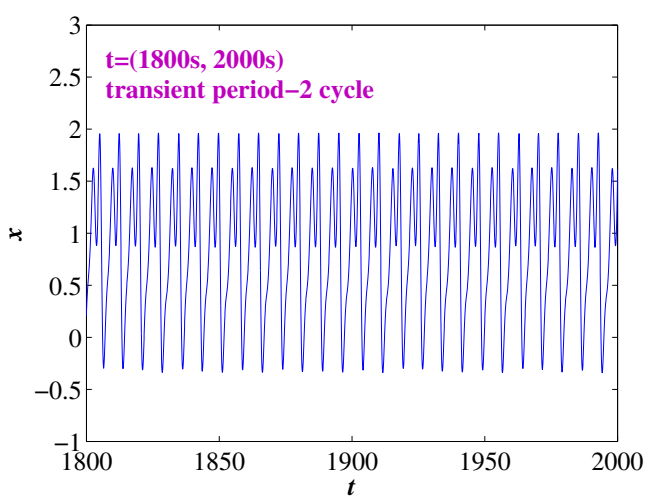

(a)

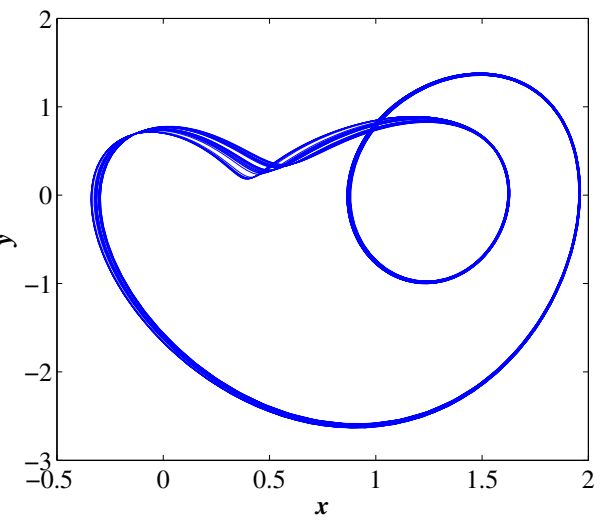

(b)

Fig. 14 (a) Time-domain plot of $t \epsilon(1800 s, 2200 s)$. (b) Phase portraits of $x-y$ axis $t \epsilon(1800 s, 2200 s)$. 


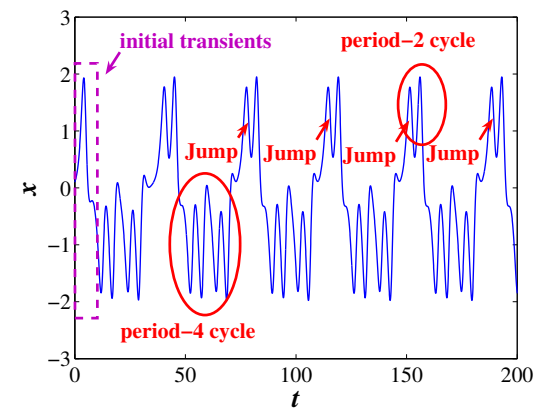

(a)

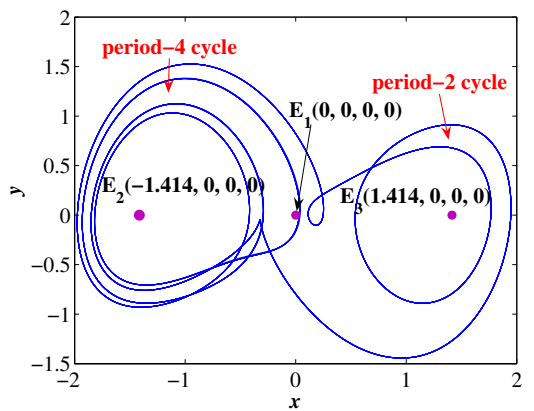

(b)

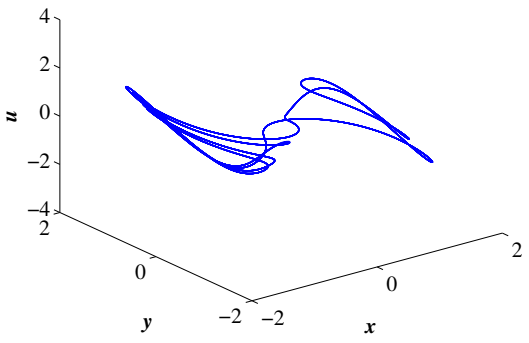

(c)

Fig. 15 After neglecting initial transients, when $t \in(20 \mathrm{~s}, 200 \mathrm{~s})$ period-4 cycle jumps to period-2 cycle (a) Time-domain plot. (b) Phase portraits of $x-y$ axis. (c) Phase portraits of $x-y-u$ axis

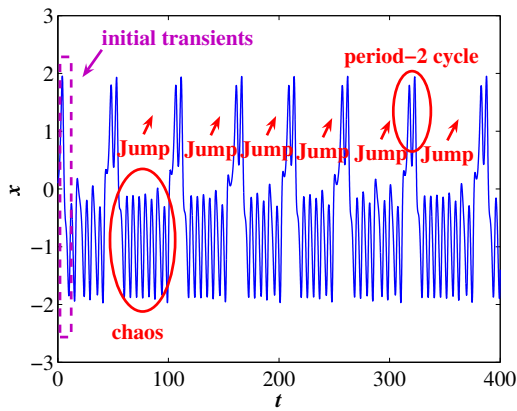

(a)

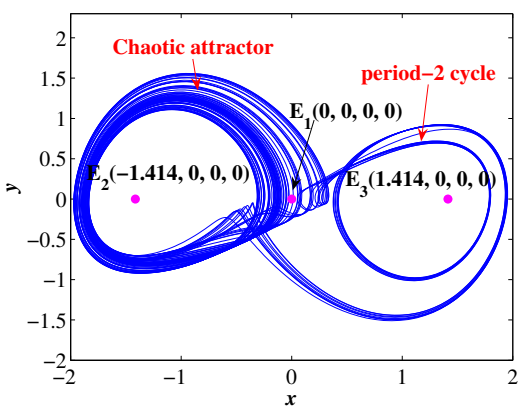

(b)

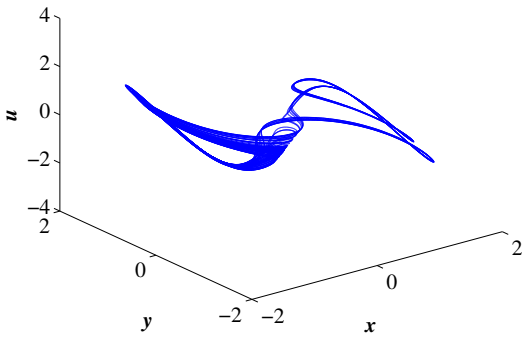

(c)

Fig. 16 After neglecting initial transients, when $t \in(20 s, 400 s)$ chaos jump to period-2 cycle (a) Time-domain plot. (b) Phase portraits of $\mathrm{x}-\mathrm{y}$ axis. (c) Phase portraits of $\mathrm{x}-\mathrm{y}-\mathrm{u}$ axis.

riod, when $t \in(1800 \mathrm{~s}, 2200 \mathrm{~s})$, It should be noted that system(7) generates period-2 cycle which is transient period(local period), not exact period(global period).

\subsubsection{State jump}

Setting parameters $\mathrm{a}=1, \mathrm{~b}=0.8, \mathrm{n}=0.2, \mathrm{~m}=1, \alpha=0.96$, the step length of simulation is 0.02, from Eq. (12), we can obtain the roots of $E_{1}$ are $\lambda_{1}=0.7378, \lambda_{2,3}=$ $0.8689 \pm 1.3984 \mathrm{i}$, so $E_{1}$ is the saddle-focus point of index 1. The roots of $E_{2,3}$ are $\lambda_{1}=-1.629, \lambda_{2,3}=0.3148 \pm 0.3148 \mathrm{i}$. From Lemma 1 , when $\alpha<0.8712$, we know $E_{2,3}$ are stable. When $\alpha>0.8712$, we know $E_{2,3}$ are saddle-focus points of index 2 . In brief, when $\alpha=0.96, E_{1}$ is the saddle-focus point of index $1, E_{2,3}$ are saddle-focus points of index 2. In Refs. [52, 54], the saddle-focus points of index 2 are important for generating chaos. Usually, scrolls are generated around the saddle focus points of index 2, and the saddle-focus points of index 1 are responsible only for connecting the scrolls.

However, from Fig. 15, it is found that the result is different from the viewpoint in Refs. [54, 55]. after neglecting some initial transients, Fig. 15(a) shows sys- tem (7) produces a new oscillation, period-4 oscillation jumps to period- 2 oscillation alternatel when $t \in(20 \mathrm{~s}$, 200s). The periodic oscillation here is asymptotic period (local period) rather than exact period. Figs. 15(b)-(c) show that period- 4 cycle and period- 2 cycle are coupled together, and the period- 4 cycle is produced by $E_{2}$, the period- 2 cycle is produced by $E_{3}$, and $E_{1}$ generates the spool to connect them. This phenomenon has not been found in other fractional-order or integer-order chaotic systems, we call it state jump.

Similarly, setting parameters $\mathrm{a}=1, \mathrm{~b}=0.8, \mathrm{n}=0.2$, $\mathrm{m}=1$ and $\alpha=0.97$, we can draw Fig. 16. Fig. 16(a) shows that chaotic oscillation jumps to period-2 oscillation alternately when $t \in(20 \mathrm{~s}, 400 \mathrm{~s})$. It is worth noting that the periodic oscillation here is transient period (local period) rather than exact period. Figs. 16(b)-(c) show that the chaotic attractor and period-2 cycle are coupled together $\mathrm{t} \in(20 \mathrm{~s}, 400 \mathrm{~s})$. The chaotic attractor is produced by $E_{2}$, numerical period- 2 cycle is produced by $E_{3}$, and $E_{1}$ generates the spool to connect them. 


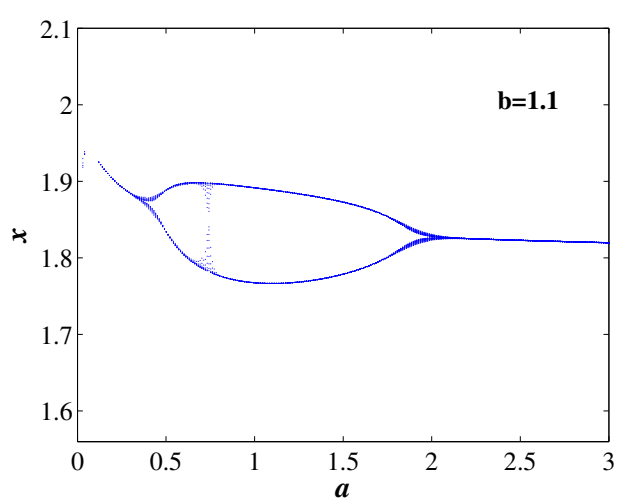

(a)

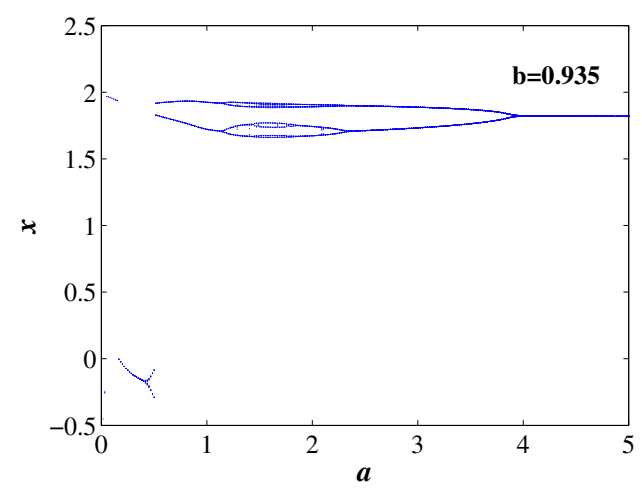

(c)

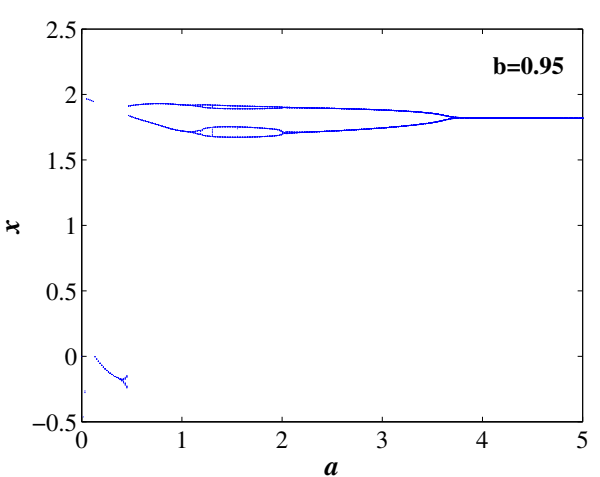

(b)

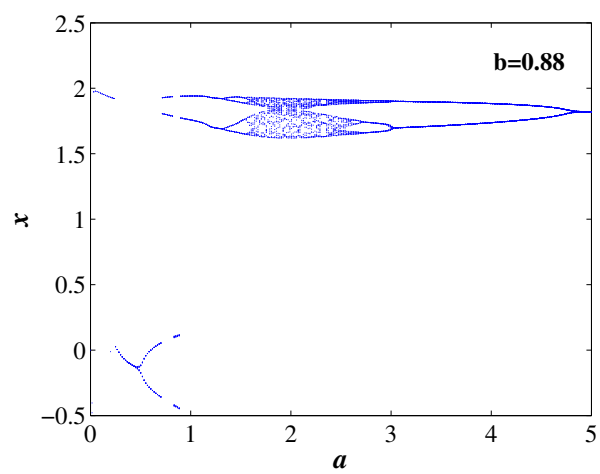

(d)

Fig. 17 Bifurcation diagrams of remerging Feigenbaum trees (a) A-period-2 bubble when b=1.1. (b) A-period-4 bubble when $\mathrm{b}=0.95$. (c) A-period-6 bubble when $\mathrm{b}=0.935$. (d) Feigenbaum tree when $\mathrm{b}=0.88$.

Table 4 The comparison with other memristive chaotic systems

\begin{tabular}{llllllll}
\hline References & Memristor & Equilibrium & Multistability & Hyperchaotic & Transient & $\begin{array}{l}\text { State } \\
\text { jump }\end{array}$ & Anti-monotonicity \\
\hline Ref. [51] & Active & Line & Multistable & No & No & No & No \\
Ref. [47] & Locally active & Three & Bi-stable & No & No & No & No \\
This paper & $\begin{array}{l}\text { Multistable } \\
\text { locally active }\end{array}$ & Infinite & Multistable & No & Alternately & Yes & Yes \\
\hline
\end{tabular}

\subsubsection{Anti-monotonicity}

Interestingly, system (7) displays a full Feigenbaum tree. In other words, system (7) is anti-monotonicity. To prove this phenomenon, we can set parameters $\mathrm{n}=0.2, \mathrm{~m}=1$, $\alpha=0.96$ and initial value $(0.1,0.1,-0.1,0)$. Parameter $\mathrm{b}$ is given as $1.1,0.95,0.935,0.88$, and a is control parameter. The corresponding bifurcation diagrams are shown in Figs. 17(a)-(d). System (7) generates the primary A-period-2 bubble, when $b=1.1$. System (7) generates the primary A-period- 4 bubble, when $b=0.95$. System (7) generates the primary A-period-6 bubble, when b $=0.935$. System (7) generates a full Feigenbaum tree, when $\mathrm{b}=0.88$.
3.4.8 Comparison with other memristive chaotic systems

Many integer-order or fractional-order chaotic systems have been studied. To clarify the characteristics of the fractional-order multistable locally active memristive chaotic systems, we compare them with the ones in other papers. Table 4 shows the comparison results. From Table 4, the fractional-order system proposed in this paper not only has complex dynamics such as infinitely many discrete equilibrium points, multistability, anti-monotonicity but also presents two phenomena which have not been found in other chaotic systems: transient transition and state jump. 
Table 5 Fractional-order capacitance $C_{q}$

\begin{tabular}{lllllll}
\hline $\mathrm{q}$ & $R_{1}(\Omega)$ & $R_{2}(\Omega)$ & $R_{3}(\Omega)$ & $C_{(x, y, z, u) 1}(\mu)$ & $C_{(x, y, z, u) 2}(\mu)$ & $C_{(x, y, z, u) 3}(\mu)$ \\
\hline 0.9 & $68.84 \mathrm{M}$ & $250 \mathrm{k}$ & 2.5 & 1.232 & 1.835 & 1.1 \\
0.97 & $87.0129 \mathrm{M}$ & 85 & - & 1.0197 & 3.8042 & - \\
0.98 & $91.187 \mathrm{M}$ & 190.933 & - & 0.97582 & 3.68059 & - \\
0.987 & $94.062 \mathrm{M}$ & 397.646 & - & 0.9458 & 3.59924 & - \\
\hline
\end{tabular}

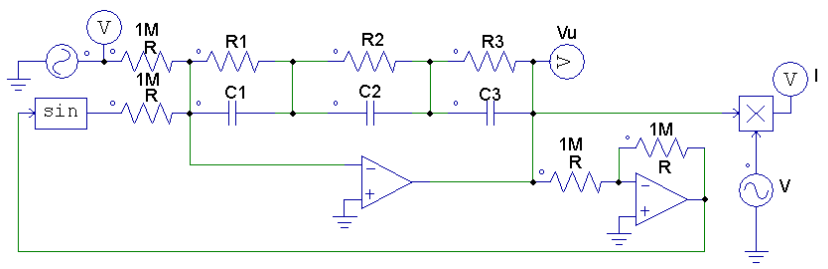

Fig. 18 Circuit configuration of the memristor

\section{Circuit implementation}

Fractional-order capacitance is the key to implement the fractional-order memristor and fractional-order chaotic circuit. In Refs. [60, 61], the fractional-order capacitance $C_{q}$ can be realized by the paralleled capacitance and resistance. See Table 5 for details.

We can implement the memristor circuit and memristive chaotic circuit by capacitors, resistors, operational amplifiers TL082CP, analog multipliers AD633JN, and trigonometric function converters AD639AD.

Different initial values can be obtained by constantly switching the supply voltage of the hardware circuit [7, 56]. However, in this paper, these special initial values are difficult to obtain precisely in hardware circuits by this method. To perfectly and accurately reveal the multistable characteristic and complex transient behavior depending on the initial conditions, PSIM Version 9.0.03 software is employed for circuit simulation.

4.1 Fractional-order multistable locally active memristor circuit implementation

From Eq. (2), the memristor circuit can be implemented. Fig. 18 shows this memristor circuit schematic diagram. According to Kirchhoff's law, the circuital equations of the memristor can be described as follows:

$\left\{\begin{array}{l}c_{q} \frac{d^{q} v_{u}}{d t^{q}}=\frac{-v}{R}+\frac{\sin \left(v_{u}\right)}{R} \\ i=v_{u} v\end{array}\right.$

where $\mathrm{q}$ is the fractional-order, $v_{u}$ is memristor state, $\mathrm{v}$ and $i$ are the input voltage and input current.

When $\mathrm{v}=\sin (6.28 \pi \mathrm{t})$, the pinched hysteresis loops shown in Fig. 19 can be obtained from the memristor circuit in PSIM simulation when q is fixed as 0.9, 0.98.

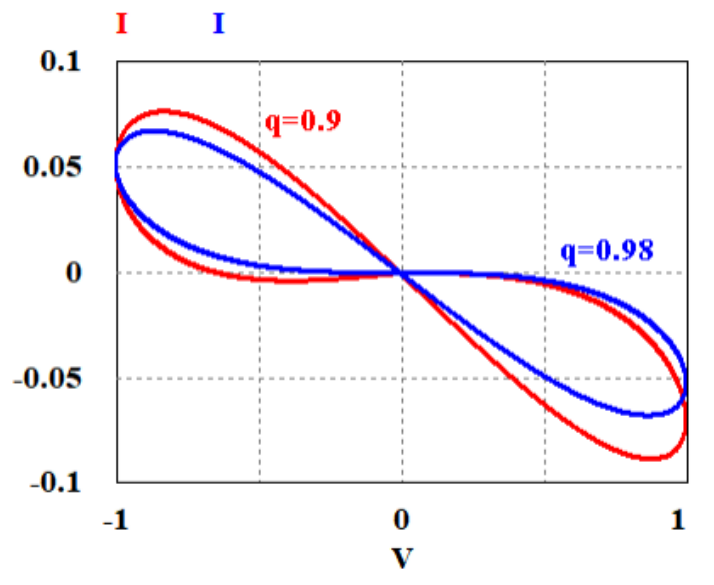

Fig. 19 Pinched hysteresis loops obtained from the memristor circuit in PSIM simulation when $\mathrm{q}=0.98$ (blue curve) and $\mathrm{q}=0.9$ (red curve).

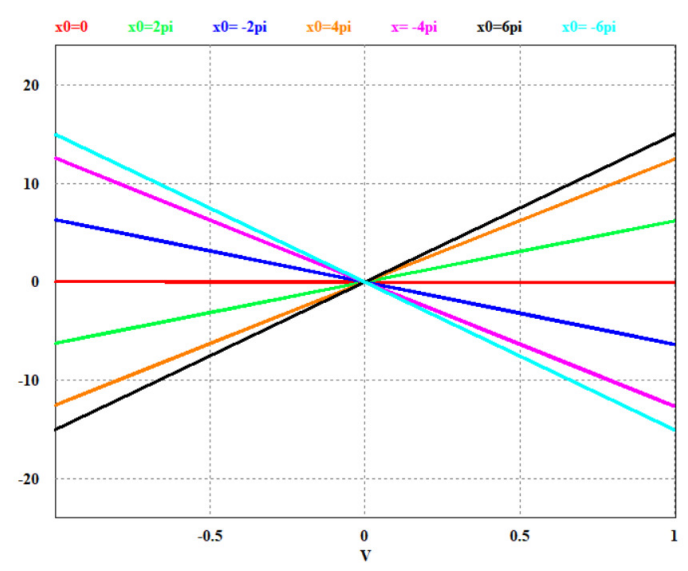

Fig. 20 Coexisting pinched hysteresis loops obtained from the memristor circuit in PSIM simulation under different initial capacitor voltages $\pm 6 \pi, \pm 4 \pi, \pm 2 \pi, 0$.

We can see Fig. 19 is consistent with Fig.1. The initial value of the circuit is determined by the initial voltage of the integrating capacitor [35, 38]. According to the voltage division formula of parallel capacitors, we know that the initial voltage of the fractional-order integrating capacitor $C_{q}$ in Fig. 18 is equal to the sum of the initial voltages of the capacitors $C_{1}, C_{2}$ and $C_{3}$. Therefore, the initial voltage of capacitors $C_{2}$ and $C_{3}$ are always fixed at $0 \mathrm{~V}$, the initial voltages of the fractional-order capacitor $C_{q}$ is set by assigning the initial 


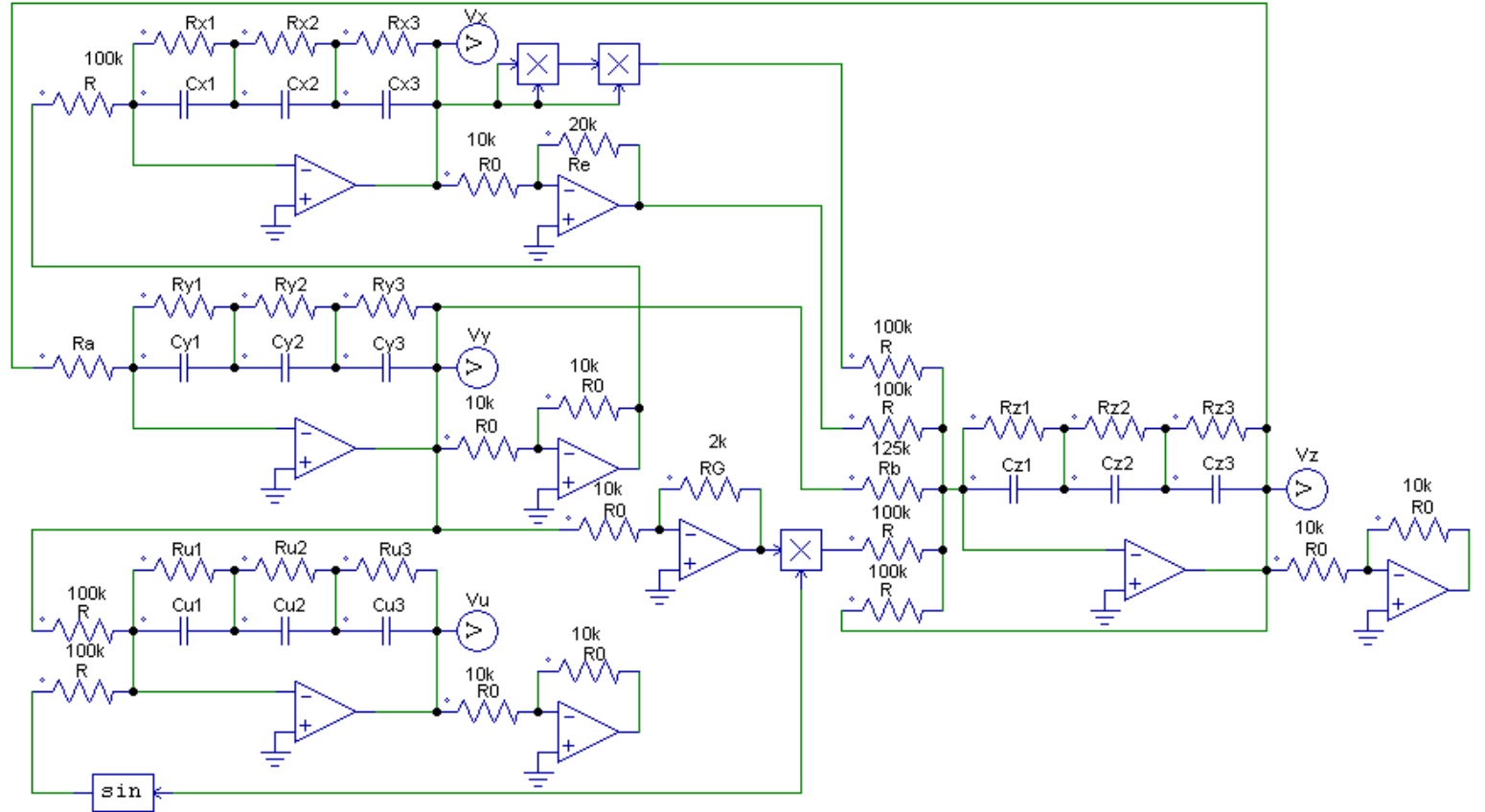

Fig. 21 Circuit configuration of the fractional-order multistable locally active memristive chaotic system

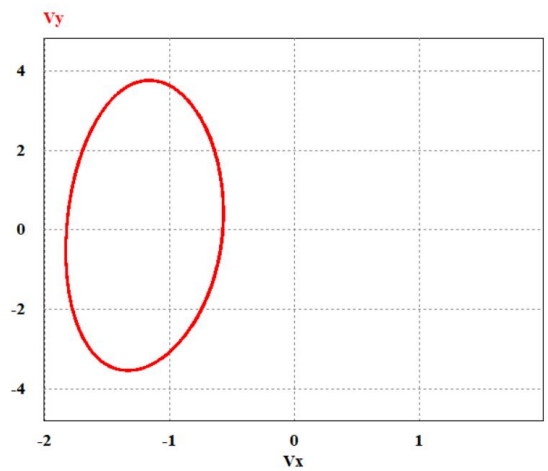

(a)

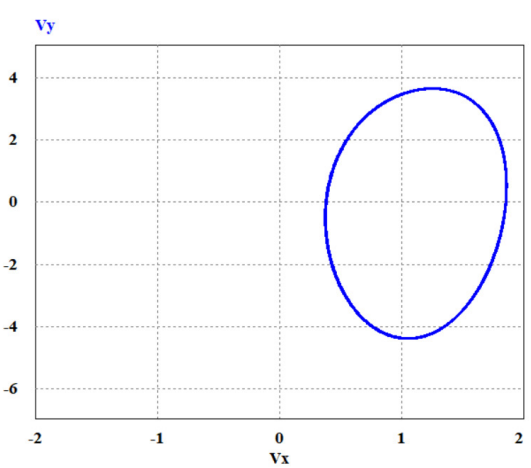

(b)

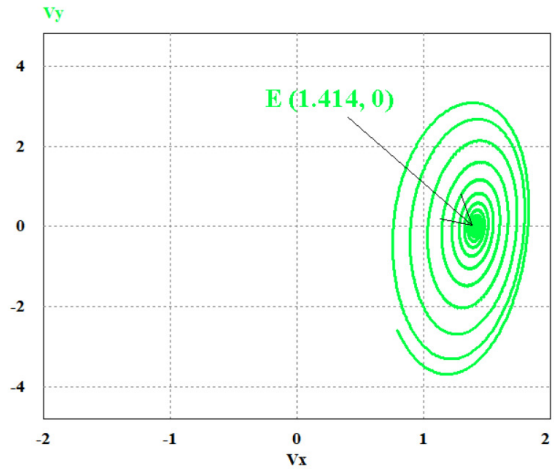

(c)

Fig. 22 Coexisting attractors obtained from the fractional-order chaotic system circuit in PSIM simulation for different initial values and $\mathrm{q}=0.9$. (a) Initial values $(0.1,-0.1,-0.1,-0.1)$. (b) Initial values $(0.1,0.1,-0.1,0)$. (c) Initial values $(0.1,0.1,-0.1,-7)$.

voltage to capacitor $C_{1}$, thus the initial value of the circuit shown in Fig.18 is set.

Setting the initial voltages of fractional-order capacitor $C_{1}$ as $-6 \pi \mathrm{V},-4 \pi \mathrm{V},-2 \pi \mathrm{V}, 0 \mathrm{~V}, 2 \pi \mathrm{V}, 4 \pi \mathrm{V}$ and $6 \pi \mathrm{V}$, the coexisting pinched hysteresis loops can be seen in Fig. 20, which are consistent with Fig.4. So the simulation results are correct.

4.2 Fractional-order multistable locally active memristive chaotic system circuit implementation

From Eq. (7), the fractional-order memristive chaotic system circuit can be implemented. Fig. 21 shows the fractional-order chaotic system circuit schematic dia- gram. According to Kirchhoff's law, the circuital equations can be described as follows:

$\left\{\begin{array}{l}c_{q} \frac{d^{9} v_{x}}{d t^{q}}=\frac{v_{y}}{R} \\ c_{q} \frac{d^{q} v_{y}}{d t^{q}}=\frac{v_{z}}{R a} \\ c_{q} \frac{d^{q} v_{z}}{d t^{q}}=-\frac{v_{x}^{3}}{R}+\frac{R_{0} v_{x}}{R}-\frac{v_{y}}{R_{b}}+\frac{R_{G} v_{z} v_{u}}{R}-\frac{v_{z}}{R} \\ c_{q} \frac{d^{q} v_{u}}{d t^{q}}=-\frac{v_{y}}{R}+\frac{\sin \left(v_{u}\right)}{R}\end{array}\right.$

Assuming that $v_{x}(0), v_{y}(0), v_{z}(0)$, and $v_{u}(0)$ are the initial voltages of $C_{x 1}, C_{y 1}, C_{z 1}, C_{u 1}$ shown in Fig. 21, other capacitors are always fixed at $0 \mathrm{~V}$. the initial voltages of the fractional-order capacitor is set by assigning the initial voltage to capacitor $C_{x 1}, C_{y 1}, C_{z 1}, C_{u 1}$, thus the initial value of the circuit shown in Fig.21 is set. 


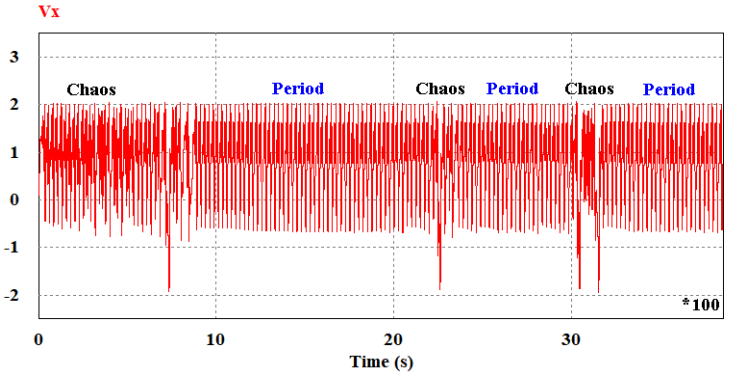

Fig. 23 The behavior of transient transition obtained from the fractional-order chaotic system circuit in PSIM simulation

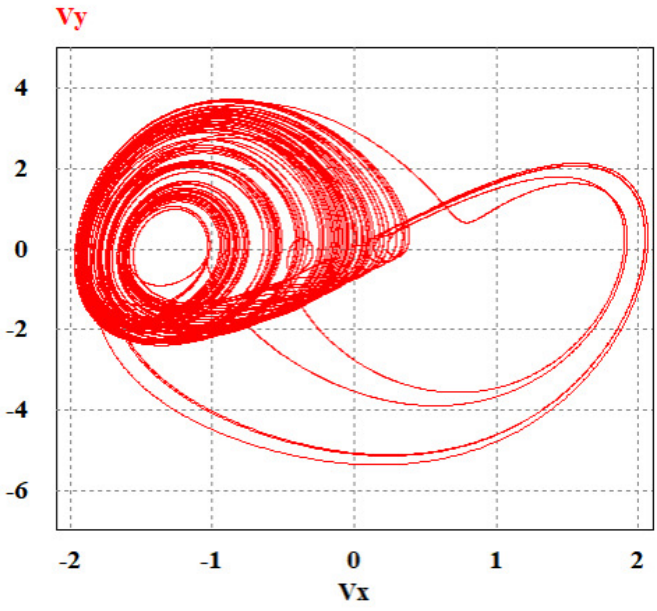

Fig. 24 The behavior of state jump obtained from the fractional-order chaotic system circuit in PSIM simulation when $\mathrm{q}=0.97$.

Setting $R_{a}=2.858 \mathrm{k} \Omega, R_{b}=125 \mathrm{k} \Omega, R_{G}=2 \mathrm{k} \Omega$, and $\mathrm{q}=0.9$, coexistieng attractors can be obtained from the fractional-order chaotic system circuit in PSIM simulation. Fig. 22(a) shows A-period-1 cycle, when $\left(v_{x}(0)\right.$, $\left.v_{y}(0), v_{z}(0), v_{u}(0)\right)=(0.1,-0.1,-0.1,-0.1)$. Fig $22(\mathrm{~b})$ shows A-period-1 cycle, when $\left(v_{x}(0), v_{y}(0), v_{z}(0), v_{u}(0)\right)=$ $0.1,-0.1,0)$. Fig $22(\mathrm{c})$ shows a stable point, when $\left(v_{x}(0)\right.$, $\left.v_{y}(0), v_{z}(0), v_{u}(0)\right)=(0.1,0.1,-0.1,-7)$. Fig. $22(\mathrm{a})-(\mathrm{c})$ are consistent with Fig. 11(a), so the simulation results are correct.

Setting $R_{a}=2.858 \mathrm{k} \Omega, R_{b}=125 \mathrm{k} \Omega, R_{G}=2 \mathrm{k} \Omega, \mathrm{q}=0.987$ and $\left(v_{x}(0), v_{y}(0), v_{z}(0), v_{u}(0)\right)=(0.1,0.1,-0.1,0)$, the behavior of transient transition can be obtained from the fractional-order chaotic system circuit in PSIM simulation. From Fig. 23, we can see the behavior of transient chaotic and transient period transition alternately occurring and Fig. 23 is consistent with Fig.12, so the simulation results are correct.

Setting $R_{a}=10 \mathrm{k} \Omega, R_{b}=125 \mathrm{k} \Omega, R_{G}=2 \mathrm{k} \Omega, \mathrm{q}=0.97$ and $\left(v_{x}(0), v_{y}(0), v_{z}(0), v_{u}(0)\right)=(0.1,0.1,-0.1,0)$, the behavior of state jump can be obtained from the fractionalorder chaotic system circuit in PSIM simulation. And
Fig. 24 is consistent with Fig.16(b), so the simulation results are correct.

\section{Conclusion}

In this paper, a fractional-order multistable locally active memristor is proposed for the first time, which has infinitely many coexisting pinched hysteresis loops under different initial states and wide locally active regions. It is found that this fractional-order memristor has stronger locally active and memory characteristics and larger nonvolatile ranges than integer-order memristor. The fractional-order chaotic system based on the fractional-order multistable locally active memristor is explored. The stability of equilibrium points and the effect of $\alpha$-order on equilibrium points are analyzed. It is found that oscillations occur only within the locally active region. By bifurcation analysis and Lyapunov exponent spectrum analysis, it is found that the system has extremely rich dynamics such as infinitely many discrete equilibrium points, multistability, antimonotonicity. Interestingly, the system presents two phenomena that have not been found in other chaotic systems, which are state jump and transient transition. Finally, the circuit simulation of the fractional-order multistable locally active memristive chaotic system using PSIM is carried out to verify the validity of the numerical simulation results.

\section{Acknowledgment}

This work is supported by the Major Research Plan of the National Natural Science Foundation of China (No.91964108), the National Natural Science Founda(Qtion of China (No.61971185) and Natural Science Foundation of Hunan Province (2020JJ4218)

\section{Compliance with ethical standards}

Conflict of interest The authors declare that they have no conflicts of interest.

\section{References}

1. Petrá , I.: A note on the fractional-order Chua's system. Chaos Solitons Fractals. 38(1), 140-147 (2008)

2. Chua, L.O.: Memristor-the missing circuit element. IEEE Trans. Circuit Theory 18(5), 507-519 (1971)

3. Ma, J., Tang, J.: A review for dynamics in neuron and neuronal network. Nonlinear Dyn. 89(3), 1569-1578 (2017) 
4. Yao, W., Wang, C., Sun, Y., Zhou, C., Lin, H..: Exponential multistability of memristive Cohen-Grossberg neural networks with stochastic parameter perturbations. Applied Mathematics and Computation. 386, 125483 (2020)

5. Lin, H ., Wang, C ., Tan, Y .: Chaotic dynamics in a neural network with different types of external stimuli. Communications in Nonlinear Science and Numerical Simulation, 90, 105390 (2020)

6. Lin, H ., Wang, C ., Tan, Y .: Hidden extreme multistability with hyperchaos and transient chaos in a Hopfield neural network affected by electromagnetic radiation. Nonlinear Dyn. 90, 2369-2386 (2020)

7. Deng, Q ., Wang, C ., Yang, M .: Four-wing hidden attractors with one stable equilibrium point, Int. J. Bifurc. Chaos. 30(6), 2050086 (2020)

8. Wang $\mathrm{C}$., Hu, X ., Zhou, L .: A Memristive Hyperchaotic Multiscroll Jerk System with Controllable Scroll Numbers[J]. Int. J. Bifurc. Chaos, 27(6), 1750091 (2017)

9. Wu, R .,Wang, C .: A new simple chaotic circuit based on memristor. Int. J. Bifurc. Chaos. 26(a10), 1650145 (2016)

10. Cheng, G ., Wang, C ., Xu, C .: A novel hyper-chaotic image encryption scheme based on quantum genetic algorithm and compressive sensing. Multimedia Tools and Applications, 79(39), 29243-29263 (2020)

11. Deng, J., Zhou, M., Wang, C., Wang, S., Xu, C.: Image segmentation encryption algorithm with chaotic sequence generation participated by cipher and multi-feedback loops. Multimedia Tools and Applications, 1-20 (2021).

12. Xu, C ., Sun, J ., Wang, C .: An Image Encryption Algorithm Based on Random Walk and Hyperchaotic Systems. Int. J. Bifurc. Chaos, 30(4), 2050060 (2020)

13. Hong, Q ., Yan, R ., Wang, C ., Sun, J .: Memristive circuit implementation of biological nonassociative learning mechanism and its applications.IEEE Transactions on Biomedical Circuits and Systems. 14(5), 1036-1050. (2020)

14. Hong, Q ., Shi, Z ., Sun, J., Du, S .: Memristive selflearning logic circuit with application to encoder and decoder. Neural Computing and Applications. 1-13, (2020)

15. Coopmans, C., Petráŝ, I., Chen, Y .: "Analogue fractional-order generalized memristive devices." International Design Engineering Technical Conferences and Computers and Information in Engineering Conference. 49019, (2009).

16. Petrá $\hat{s}$, I .: "Fractional-order memristor-based Chua's circuit." IEEE Transactions on Circuits and Systems II: Express Briefs 57(12) 975-979 (2010)

17. Cafagna, D., Grassi, G.: On the simplest fractional-order memristor-based chaotic system. Nonlinear Dyn. 70(2), 1185-1197 (2012)

18. Teng, L., Iu, H. H., Wang, X., Wang, X.: Chaotic behavior in fractional-order memristor-based simplest chaotic circuit using fourth degree polynomial. Nonlinear Dyn, 77(12), 231-241 (2014)

19. Si, G ., Diao, L ., Zhu, J .: "Fractional-order chargecontrolled memristor: theoretical analysis and simulation." Nonlinear Dyn. 87(4), 2625-2634 (2017)

20. Yang, N., Xu, C., Wu, C., Jia, R., Liu, C.: Fractionalorder cubic nonlinear flux-controlled memristor: theoretical analysis, numerical calculation and circuit simulation. Nonlinear Dyn. 97(1), 33-44 (2019)

21. Yu, Y., Wang, Z.: A fractional-order memristor model and the fingerprint of the simple series circuits including a fractional-order memristor. Acta Physica Sinica 64(23), 238401 (2015)

22. Yu, Y., Wang, Z.: Initial state dependent nonsmooth bifurcations in a fractional-order memristive circuit. Int. J. Bifurc. Chaos 28(07), 1850091 (2018)
23. Chua, L.O.: If it's pinched it's a memristor. Semicond. Sci. Technol. 29(10), 1-42 (2014)

24. Chua, L.O.: Local activity is the origin of complexity. Int.. J. Bifurc. Chaos 15(11), 3435-3456 (2005)

25. Corinto, F., Ascoli, A., Gilli, M.: Nonlinear dynamics of memristor oscillators. IEEE Transactions on Circuits and Systems I: Regular Papers, 58(6), 1323-1336 (2011)

26. Mainzer, k ., Chua, L.O.: The Local Activity Principle The Cause of Complexity and Symmetric Breaking, pp. 146-159, Chaos, CNN, Memristors and Beyond, London (2013)

27. Gibson, G.A., Musunuru, S., Zhang, J., Vandenberghe, K.,Lee, J., Hsieh, C.C., Stanley Williams, R .: An accurate locally active memristor model for S-type negative differential resistance in NbOx. Phys. Lett. A 108(2), 023505 (2016)

28. Weiher, M., Herzig, M., Tetzlaff, R., Ascoli, A., Mikolajick,T., Slesazeck, S.: Pattern formation with locally active S-type NbOx memristors. IEEE Trans. Circuits Syst. I Reg.Pap. 66(7), 2627-2638 (2019)

29. Chua, L.O.: Everything you wish to know about memristors but are afraid to ask. Radioengineering 24(2), 319 (2015)

30. Mannan, I., Hyuncheol, C., Hyongsuk, K.: Chua corsage memristor oscillator via hopf bifurcation, Int. J. Bifurc. Chaos. 26(04), 1630009 (2016).

31. Jin, P., Wang, G., Iu, H.H., Fernando, T.: A locally active memristor and its application in a chaotic circuit. IEEE Trans. Circuits Syst. II Exp. Briefs 65(2), 246-250 (2017)

32. Chang, H., Wang, Z., Li, Y., Chen, G.: Dynamic analysis of a bistable bi-local active memristor and its associated oscillator system. Int. J. Bifurc. Chaos 28(8), 1850105 (2018)

33. Mannan, Z. I., Yang, C., Kim, H.: Oscillation with 4lobe Chua corsage memristor. IEEE Circuits and Systems Magazine, 18(2), 14-27 (2018)

34. Dong, Y., Wang, G., Chen, G., Shen, Y., Ying, J.: A bistable nonvolatile locally-active memristor and its complex dynamics. Communications in Nonlinear Science and Numerical Simulation. 84, 105203 (2020)

35. Tan, Y., Wang, C.: A simple locally active memristor and its application in HR neurons. Chaos: An Interdisciplinary Journal of Nonlinear Science. 30(5), 053118 (2020).

36. Lin, H., Wang, C., Sun, Y., Yao, W.: Firing multistability in a locally active memristive neuron model. Nonlinear Dyn. 100(4), 3667-3683 (2020)

37. Zhu, M., Wang, C., Deng, Q., Hong, Q.:Locally Active Memristor with Three Coexisting Pinched Hysteresis Loops and Its Emulator Circuit. Int. J. Bifurc. Chaos, 30(13), 2050184 (2020).

38. Lin, H., Wang, C., Hong, Q., Sun, Y.: A multi-stable memristor and its application in a neural network. IEEE Transactions on Circuits and Systems II: Express Briefs, 67(12), 3472-3476 (2020)

39. Gorenflo, R., Mainardi, F.: Fractional calculus. In: Fractals and Fractional Calculus in Continuum Mechanics, pp. 223-276. Springer, Berlin (1997)

40. Podlubny, I.: Fractional Differential Equations: An Introduction to Fractional Derivatives, Fractional Differential Equations, to Methods of Their Solution and Some of Their Applications, vol. 198. Academic Press, London (1999)

41. Kengne, J., Njitacke, Z. T., Fotsin, H. B.: Dynamical analysis of a simple autonomous jerk system with multiple attractors. Nonlinear Dyn, 83(1-2), 751-765 (2016).

42. Ahmed, E., El-Sayed, A. M. A., El-Saka, H. A.: Equilibrium points, stability and numerical solutions of fractionalorder predator-prey and rabies models. Journal of Mathematical Analysis and Applications, 325(1), 542-553 (2007) 
43. El-Saka, H. A., Ahmed, E., Shehata, M. I., El-Sayed, A. M. A.: On stability, persistence, and Hopf bifurcation in fractional order dynamical systems. Nonlinear Dyn, 56(12), 121 (2009)

44. Tavazoei, M., Haeri, M.: A proof for non existence of periodic solutions in time invariant fractional order systems. Automatica 45, 1886-1890 (2009)

45. Kaslik, E., Sivasundaram, S.: Non-existence of periodic solutions in fractional-order dynamical systems and a remarkable difference between integer and fractional-order derivatives of periodic functions. Nonlinear Anal. Real World Appl. 13, 1489-1497 (2012)

46. Danca, M. F., Feckan, M., Kuznetsov, N. V., Chen, G.: Complex dynamics, hidden attractors and continuous approximation of a fractional-order hyperchaotic PWC system. Nonlinear Dyn, 91(4), 2523-2540 (2018)

47. Danca, M.-F., Feckan, M., Chen, G.: Impulsive stabilization of chaos in fractional-order systems. Nonlinear Dyn. 89(3), 1889-1903 (2017)

48. Wang, Y., Li, C.: Does the fractional Brusselator with efficient dimension less than 1 have a limit cycle?. Physics Letters A, 363(5-6), 414-419 (2007)

49. Kang, Y.-M., Xie, Y., Lu, J.C., Jiang, J.: On the nonexistence of non-constant exact periodic solutions in a class of the Caputo fractional-order dynamical systems. Nonlinear Dyn. 82, 1259-1267 (2015)

50. Diethelm, K., Ford, N. J., Freed, A. D.: A predictor-corrector approach for the numerical solution of fractional differential equations. Nonlinear Dyn, 29(1), 3-22 (2002)

51. Danca, M. F., Kuznetsov, N.: Matlab code for Lyapunov exponents of fractional-order systems. Int. J. Bifurc. Chaos, 28(05), 1850067 (2018)

52. Li, C., Sprott, J.C.: Multistability in the Lorenz system:a broken butterfly. Int. J. Bifurc. Chaos 24(10), 1450131(2014)

53. Parastesh, F., Jafari, S., Azarnoush, H.: Traveling patterns in a network of memristor based oscillators with extreme multistability. Eur. Phys. J. Spec. Top. 228(10), 21232131(2019)

54. Chua, L.O., Komuro, M., Matsumoto, T.: The double scroll family. IEEE transactions on circuits and systems, 33(11), 1072-1118 (1986)

55. Yu, Y., Bao, H., Shi, M., Bao, B., Chen, Y., Chen, M.: Complex Dynamical Behaviors of a Fractional-Order System Based on a Locally Active Memristor. Complexity, 2019 (2019)

56. Bao, B. C., Liu, Z., Xu, J. P.: Steady periodic memristor oscillator with transient chaotic behaviours. Electronics letters, 46(3), 237-238 (2010)

57. Bao, B., Jiang, P., Wu, H., Hu, F.: Complex transient dynamics in periodically forced memristive Chua's circuit. Nonlinear Dyn, 79(4), 2333-2343 (2015)

58. Bao, H., Wang, N., Bao, B., Chen, M., Jin, P., Wang, G.: Initial condition-dependent dynamics and transient period in memristor-based hypogenetic jerk system with four line equilibria. Communications in Nonlinear Science and Numerical Simulation, 57, 264-275 (2018)

59. Wu, J., Wang, G., Iu, H. H. C., Shen, Y., Zhou, W.:A nonvolatile fractional order memristor model and its complex dynamics. Entropy, 21(10), 955 (2019)

60. Pham, V. T., Kingni, S. T., Volos, C., Jafari, S., Kapitaniak, T.: A simple three-dimensional fractional-order chaotic system without equilibrium: Dynamics, circuitry implementation, chaos control and synchronization. AEUinternational Journal of Electronics and Communications, $78,220-227$ (2017)
61. Fu-Hong, M., Shu-Yi, S., Wen-Di, H., En-Rong, W.: Circuit implementations, bifurcations and chaos of a novel fractional-order dynamical system. Chinese Physics Letters, 32(3), 030503 (2015) 
Figures

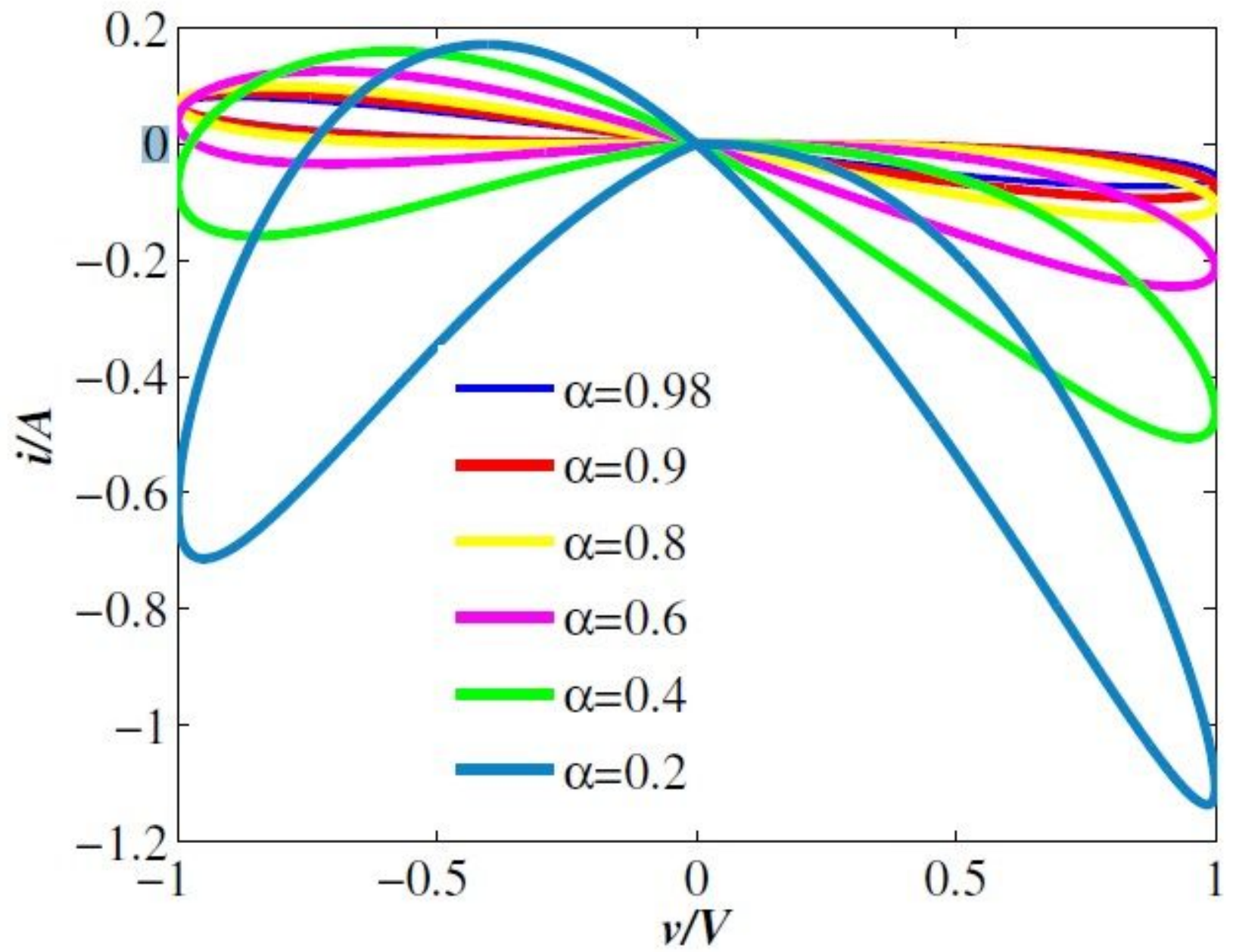

Figure 1

Pinched hysteresis loop for different a-orders, when parameters $m=1$ and $n=1$ 


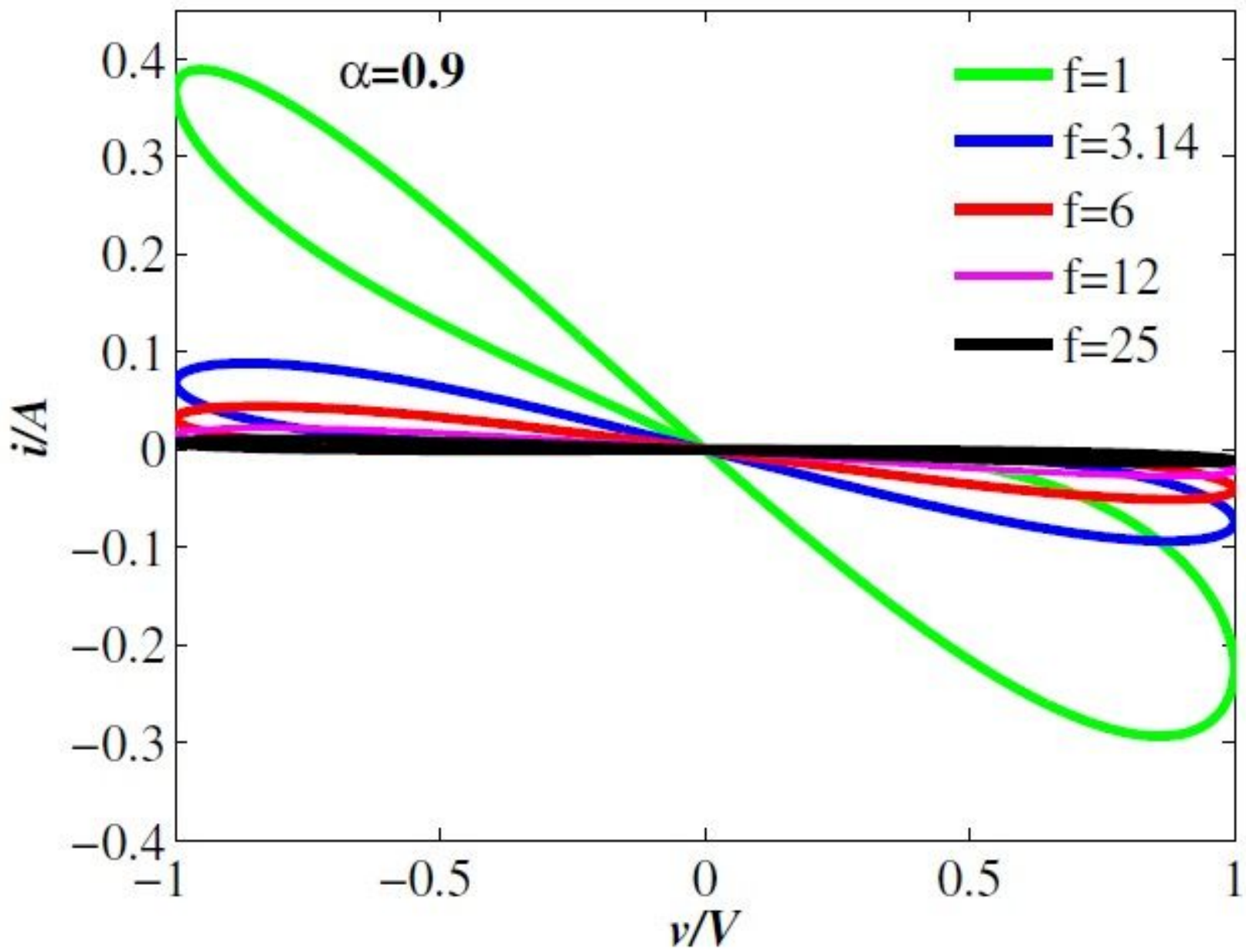

Figure 2

Pinched hysteresis loop for different frequencies, when parameters $m=1, n=1$ and $a=0.9$ 


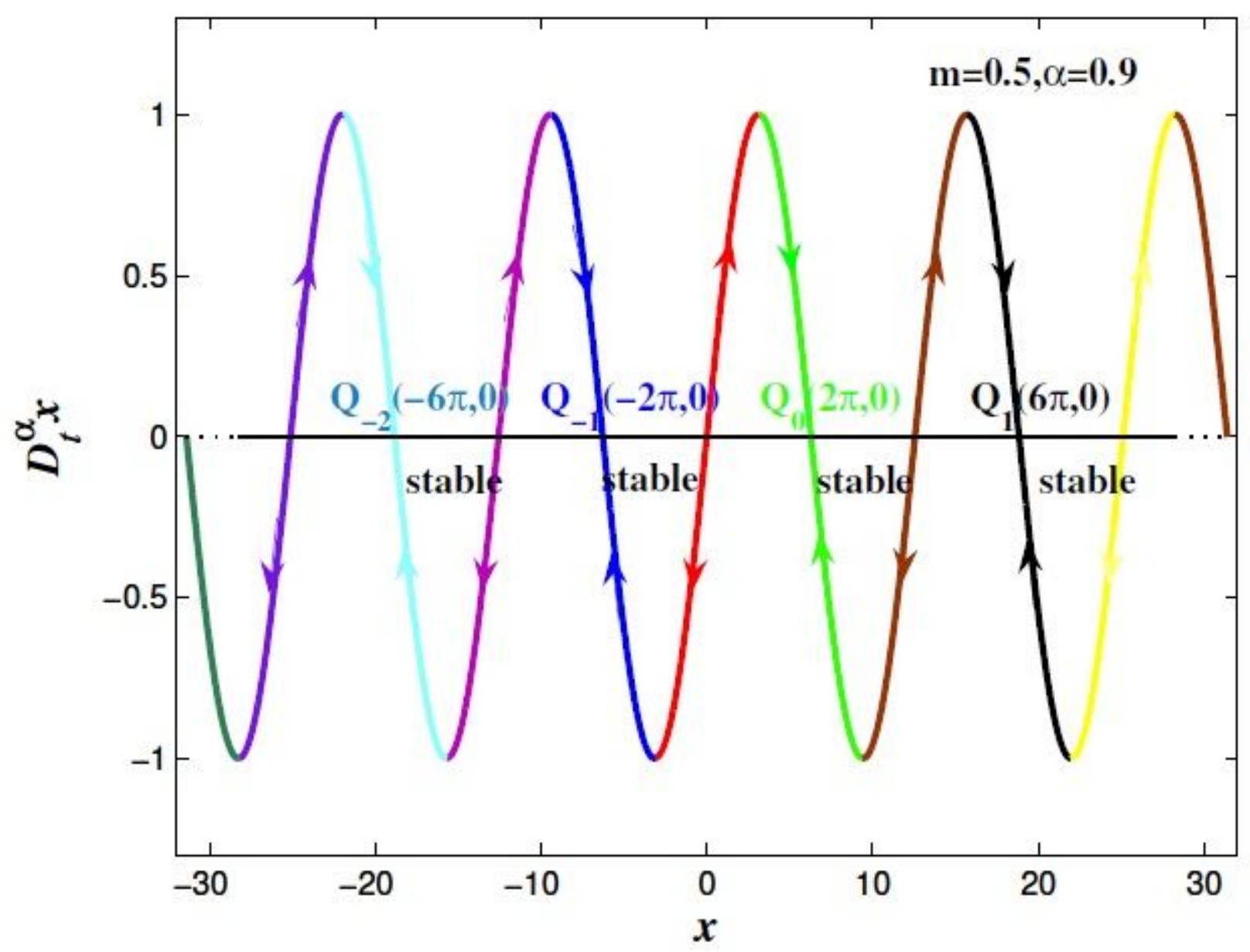

Figure 3

POP curve of the memristore, when parameters $\mathrm{m}=0.5, \mathrm{n}=1$ and $\mathrm{a}=0.9$ 


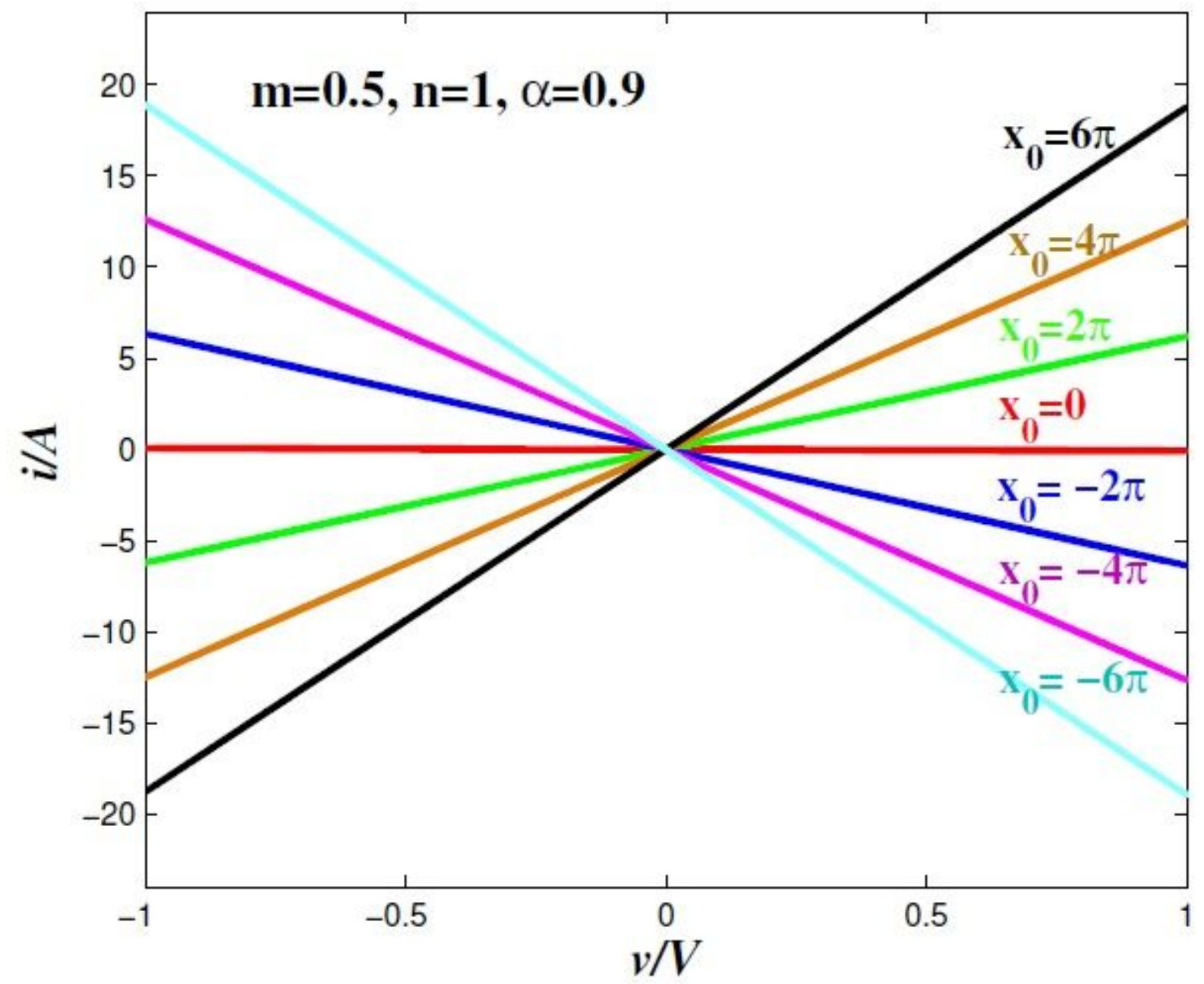

Figure 4

Coexisting Pinched hysteresis loops, , when parame- ters $m=0.5, n=1$ and $a=0.9$ 


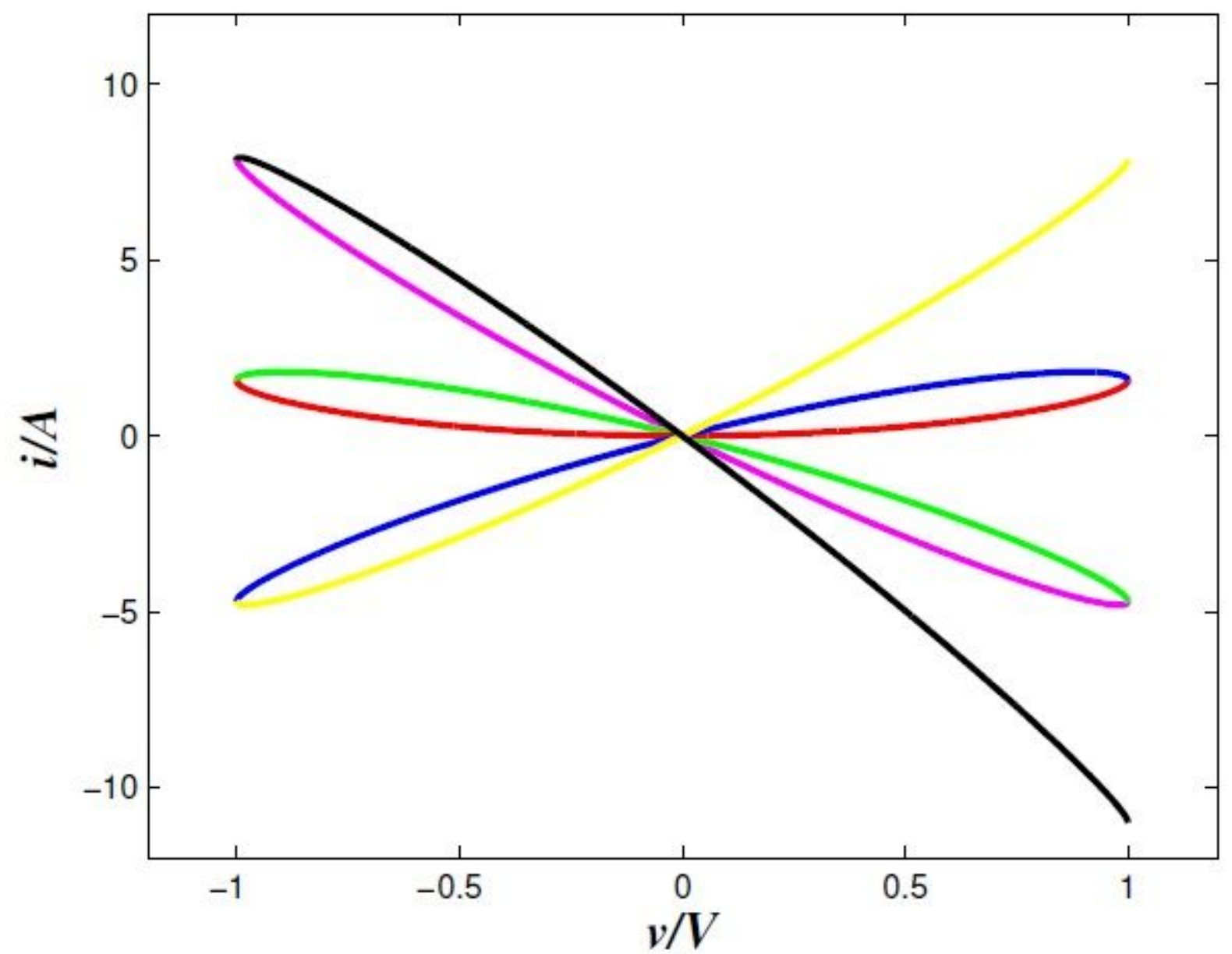

Figure 5

Locally active ranges when $\mathrm{x} 0 \mathrm{e}(-10,8]$, parameters $\mathrm{m}=0.5$ and $\mathrm{n}=1$ 


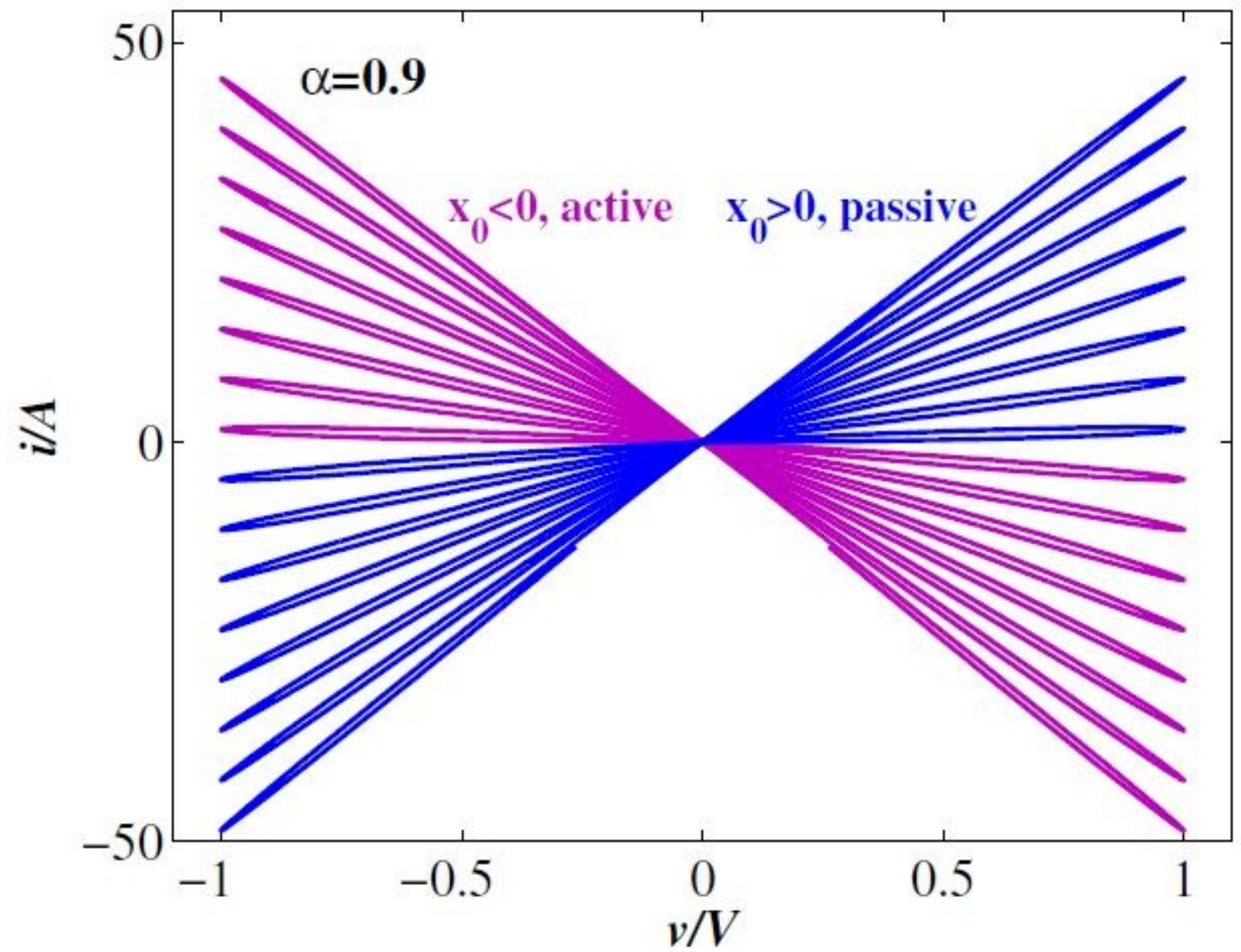

Figure 6

Locally active ranges when $x 0 e[-50,50]$, when param- eters $m=1, n=1$ and $a=0.9$

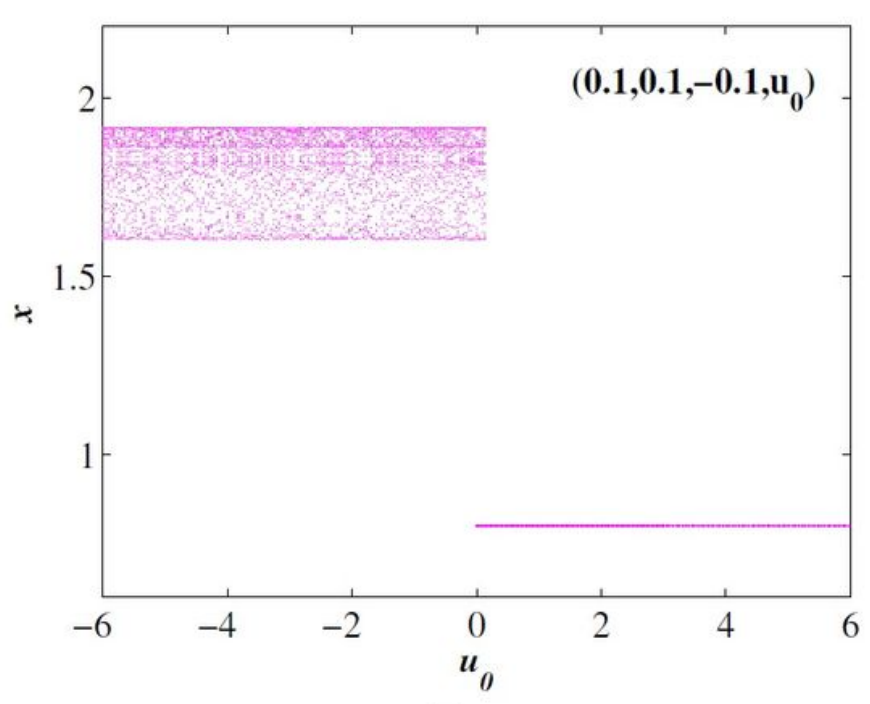

(a)

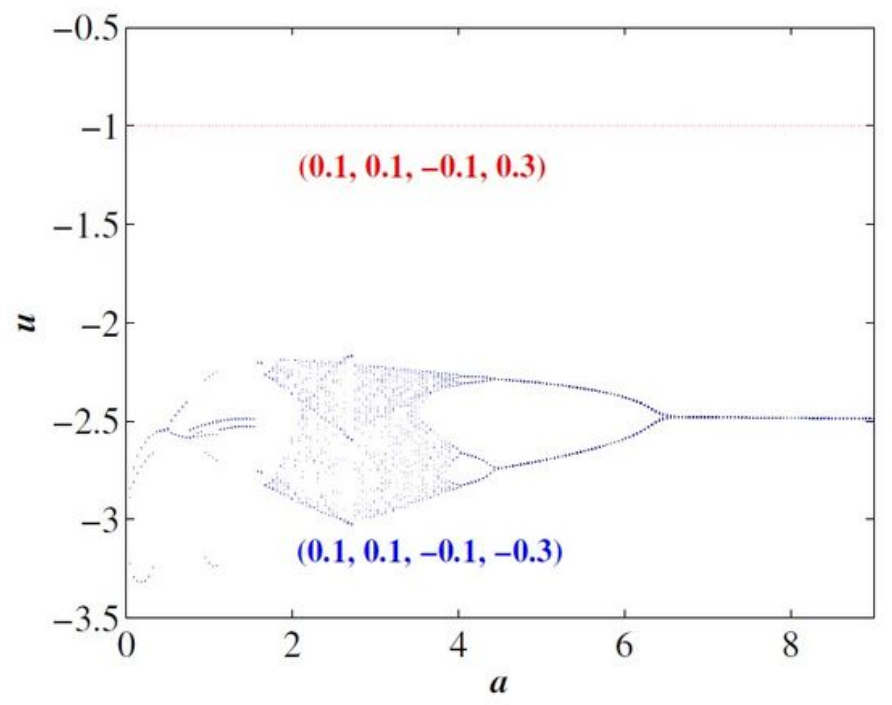

(b)

Figure 7 
(a) Bifurcation diagram for u0. (b) Bifurcation diagram for parameter a with initial value $(0.1,0.1,-0.1$, $-0.3)$ (blue curve) and $(0.1,0.1,-0.1,0.3)$ (red curve)

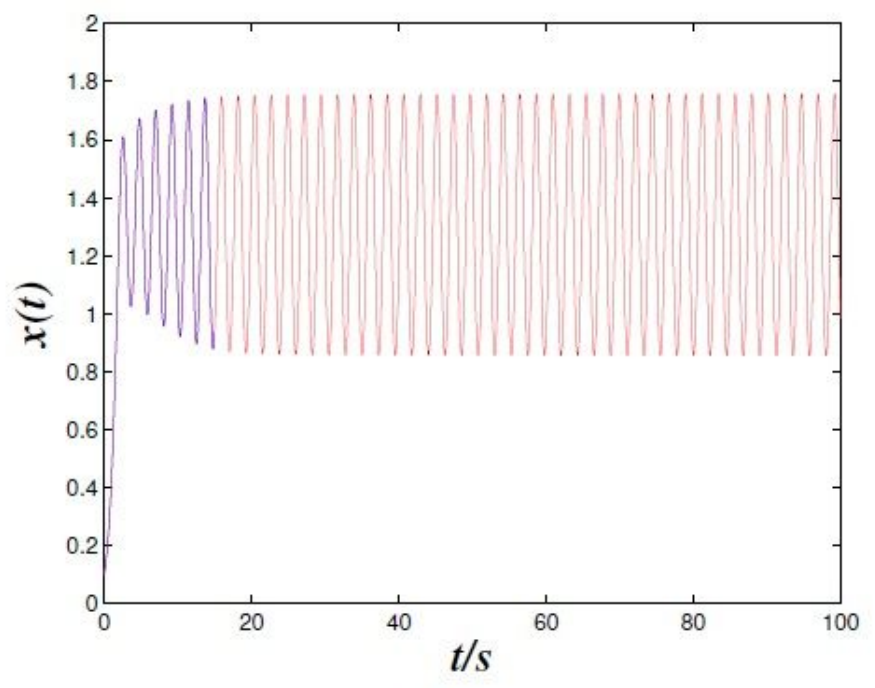

(a)

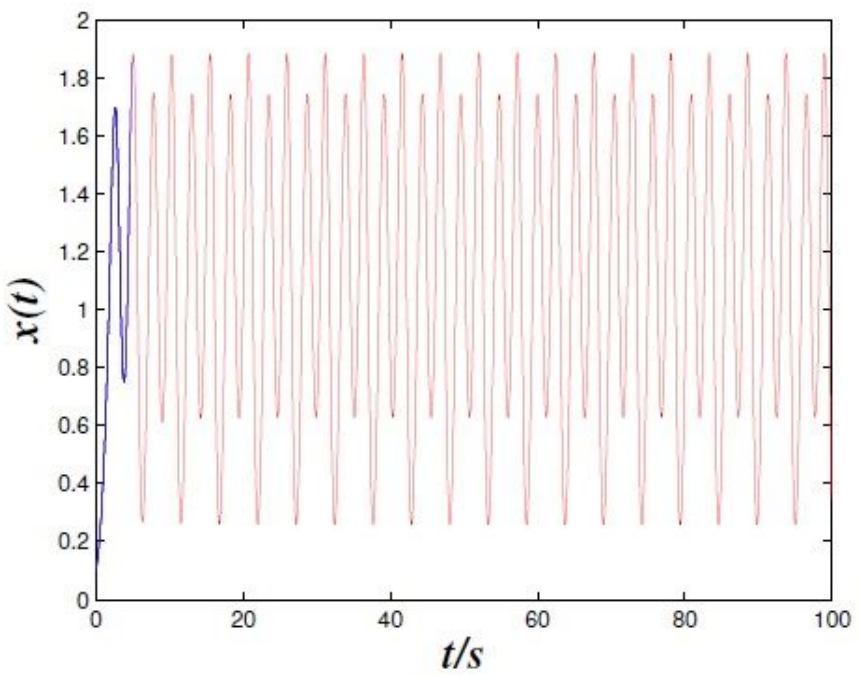

(c)

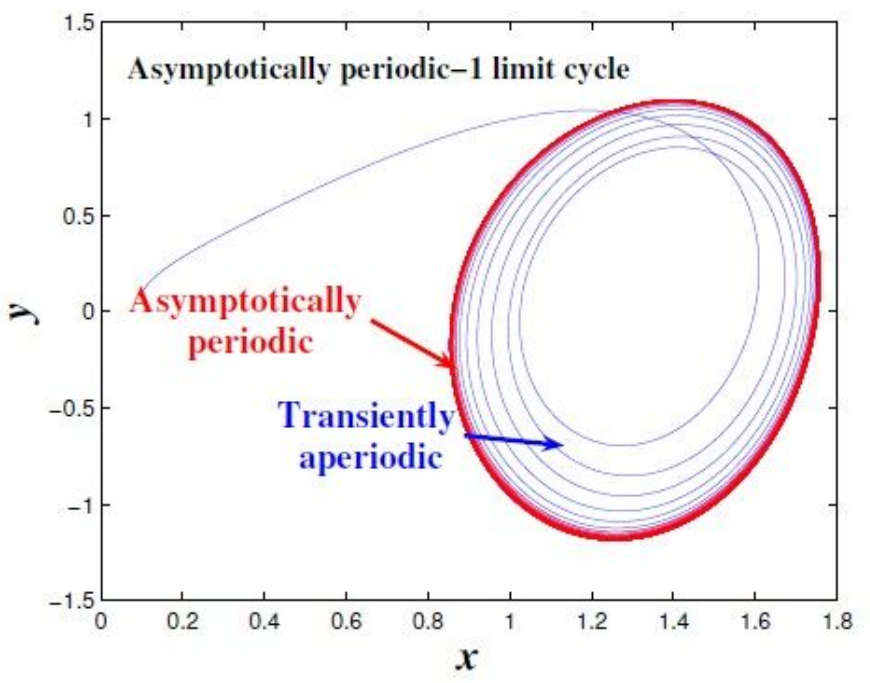

(b)

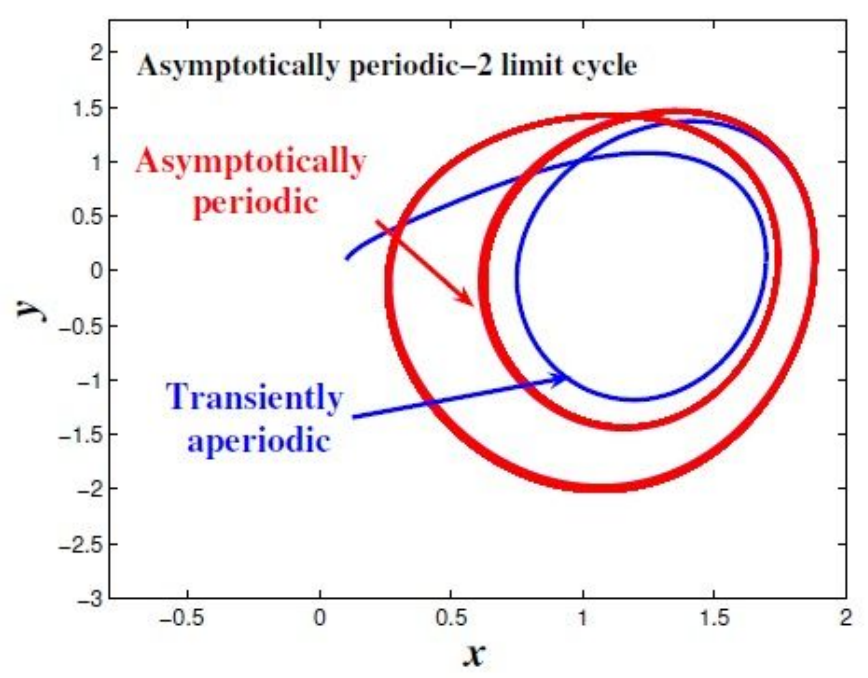

(d)

Figure 8

(a) the timing diagrams of the system (7) when a is 0.9. (b) the phase diagrams of system (7) when a is 0.9 . (c) the timing diagrams of the system (7) when a is 0.95 . (d) the phase diagrams of system (7) when a is 0.95 . 


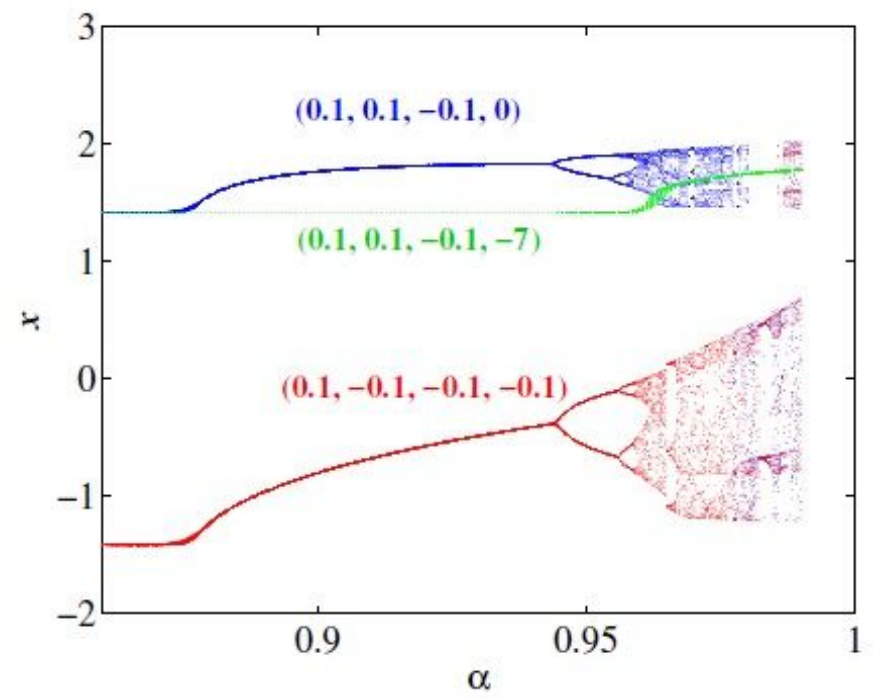

(a)

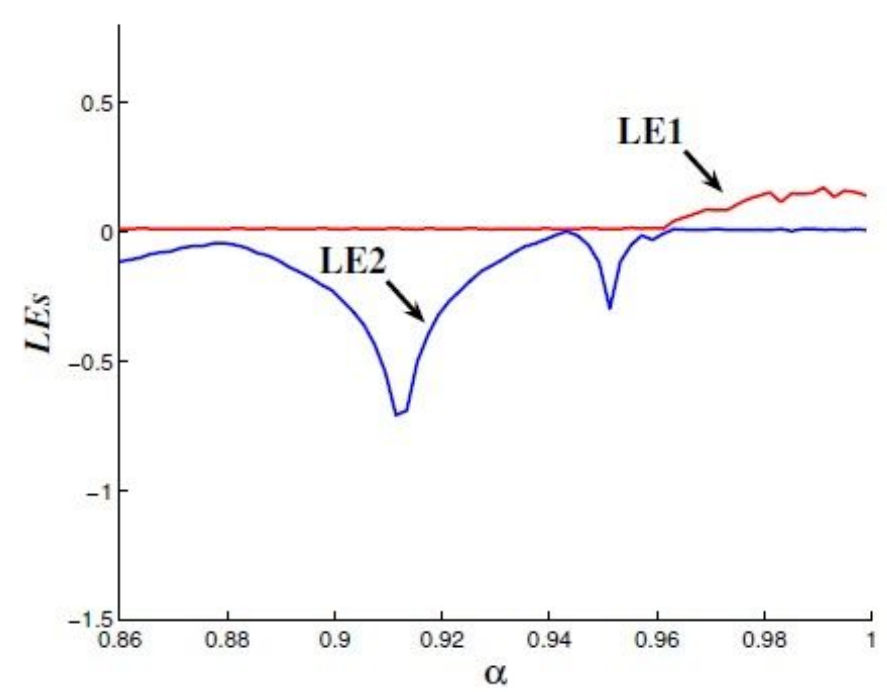

(b)

\section{Figure 9}

(a) Bifurcation diagram for a with initial values $((0.1,0.1,-0.1,0)$ (blue curve), $(0.1,-0.1,-0.1,-0.1)$ (red curve) and $(0.1,0.1,-0.1,-7)$ (green curve). (b) Lyapunov exponent spectrum for initial values $(0.1,0.1$, $-0.1,0)$.

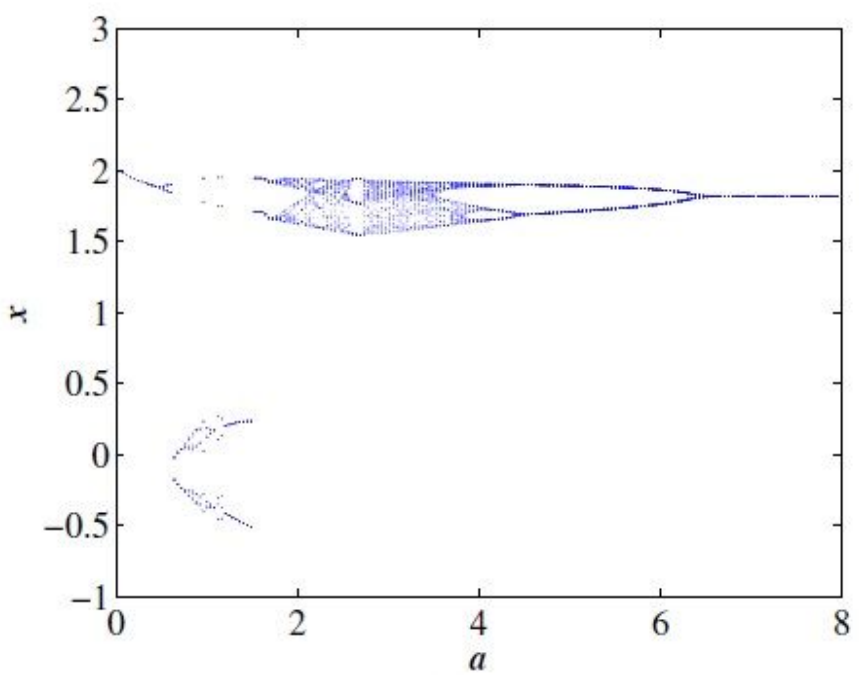

(a)

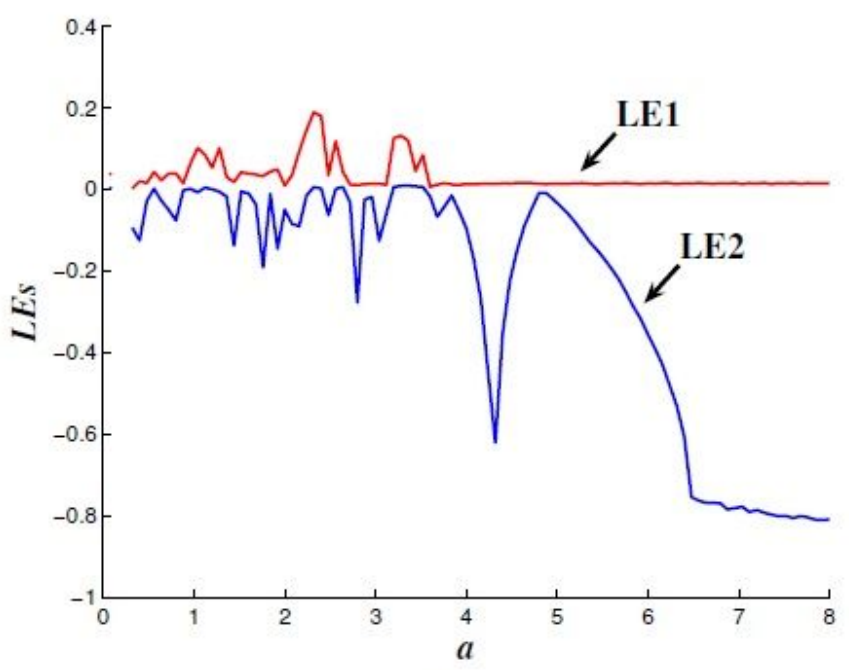

(b)

Figure 10

(a) Bifurcation diagram for parameter a with initial values $(0.1,0.1,-0.1,0)$. (b) Lyapunov exponent spectrum for parameter a with initial values $(0.1,0.1,-0.1,0)$ 


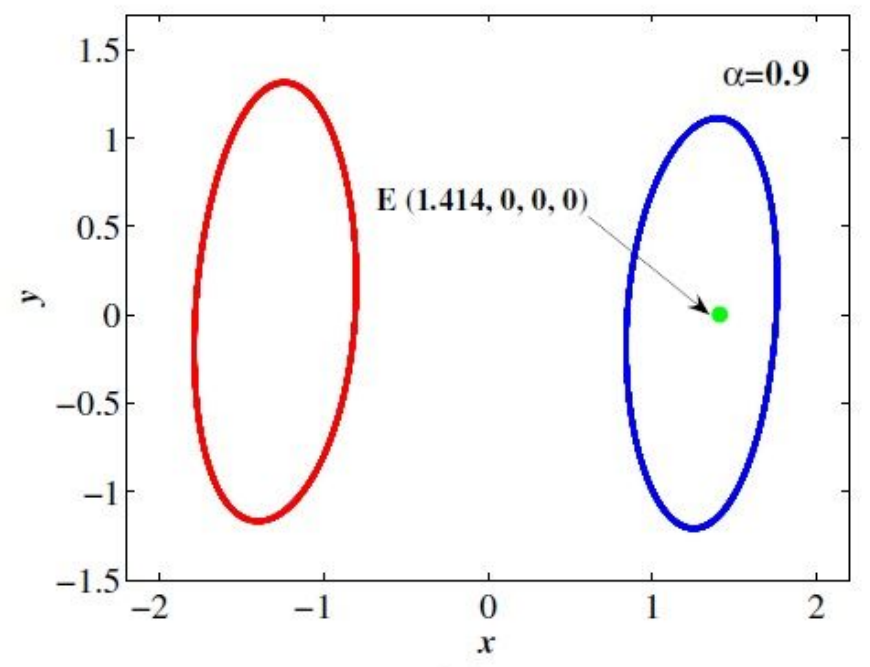

(a)

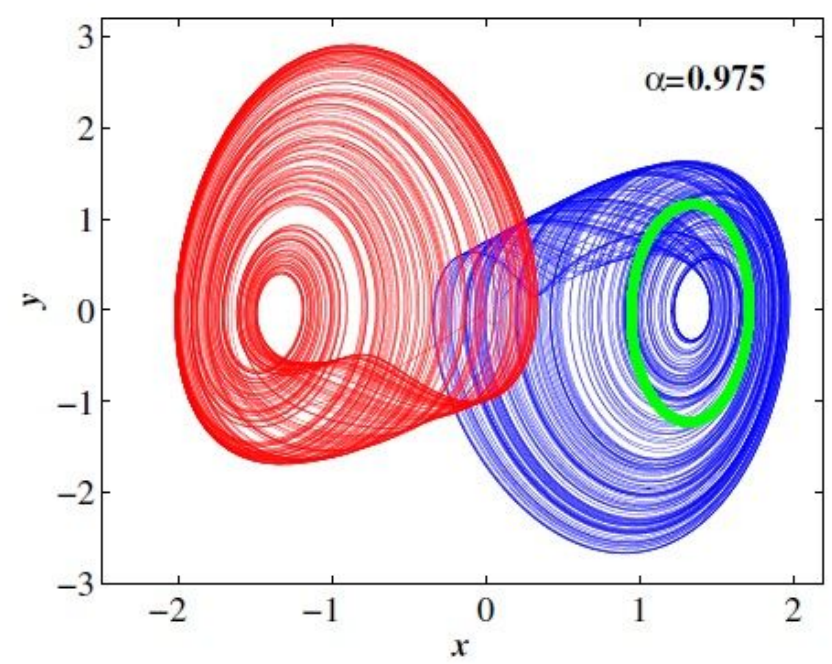

(c)

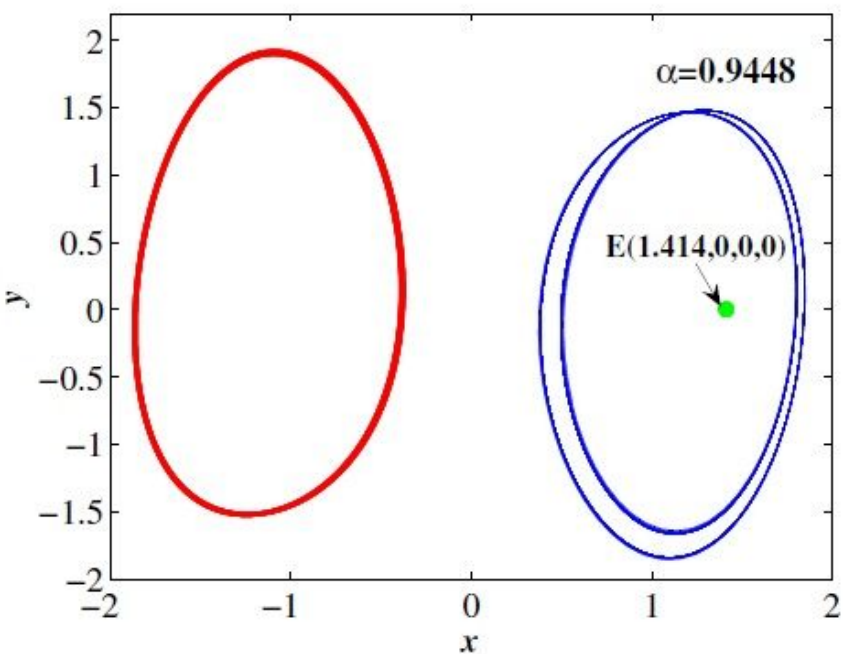

(b)

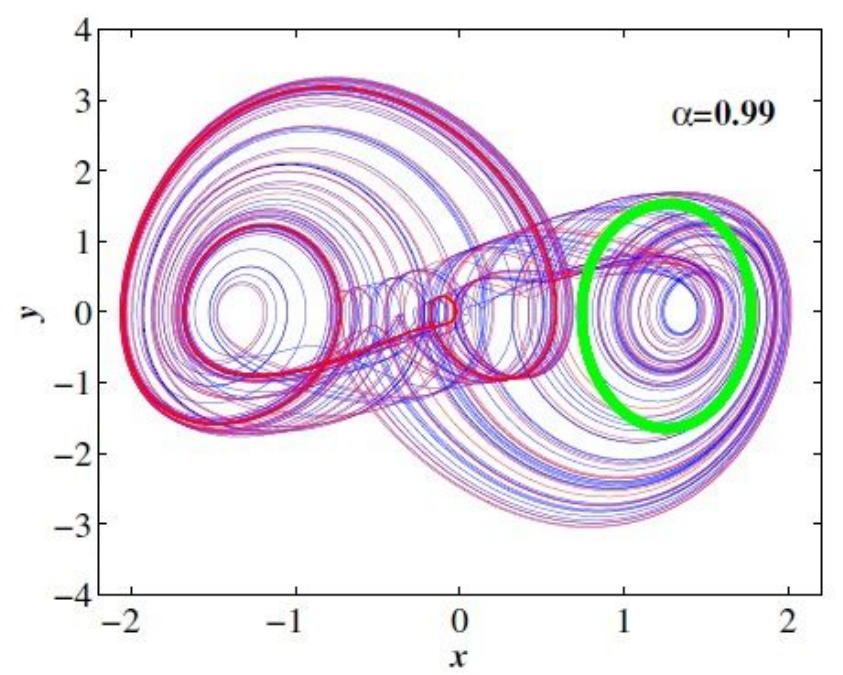

(d)

\section{Figure 11}

Phase portraits for different a with initial values $((0.1,0.1,-0.1,0)$ (blue curve), $((0.1,-0.1,-0.1,-0.1)$ (red curve) and $((0.1,0.1,-0.1,-7)$ (green curve)) (a) $a=0.9$ (b) $a=0.9448$ (c) $a=0.975$ (d) $a=0.99$ 


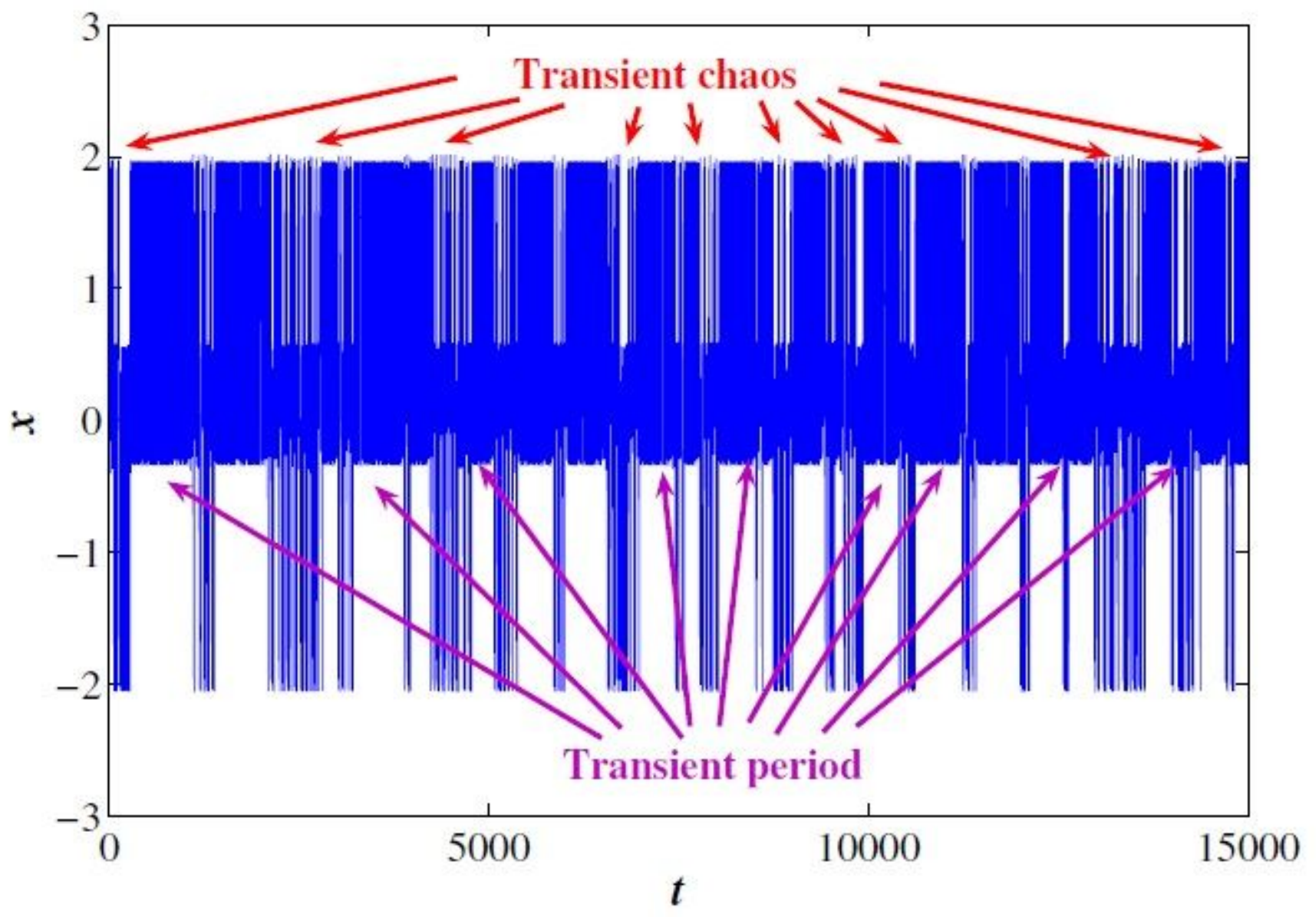

Figure 12

Time-domain plot of te(0,15000s), when $a=3.5, b=0.8, n=0.2, m=1, a=0.987$, initial value $(0.1,0.1,-0.1,0)$

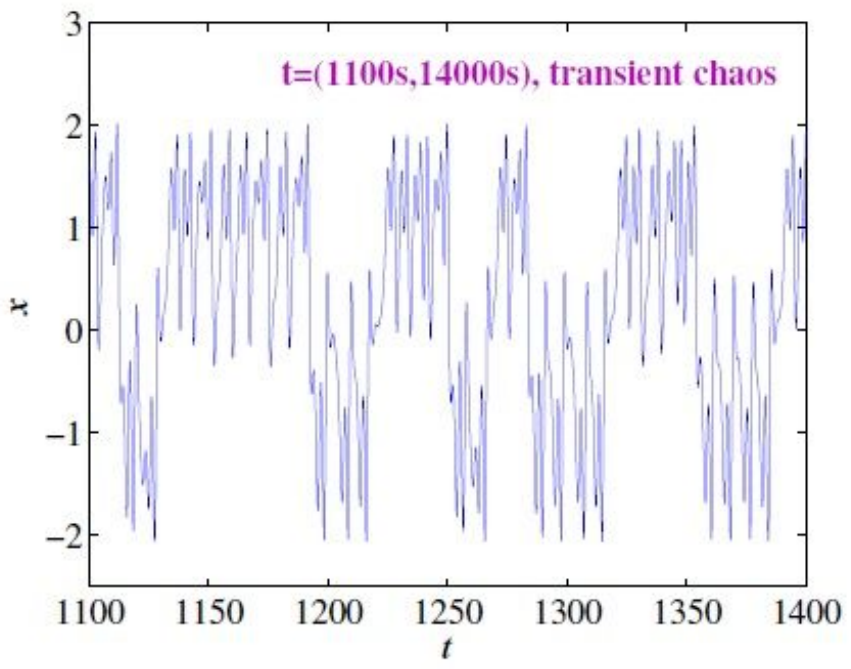

(a)

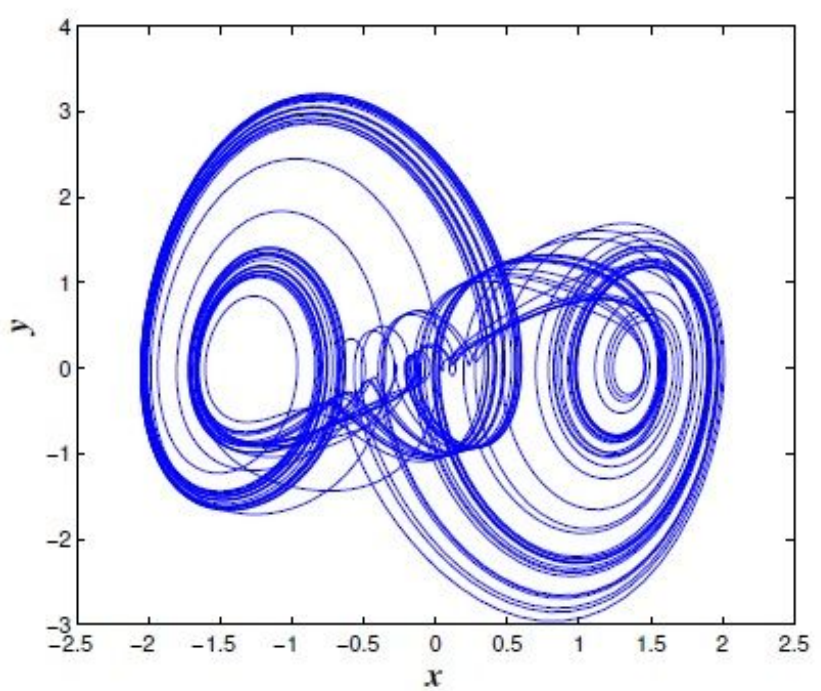

(b)

Figure 13 
(a) Time-domain plot of te(1100s, 1400s). (b) Phase portraits of $x-y$ axis te(1100s, 1400s).

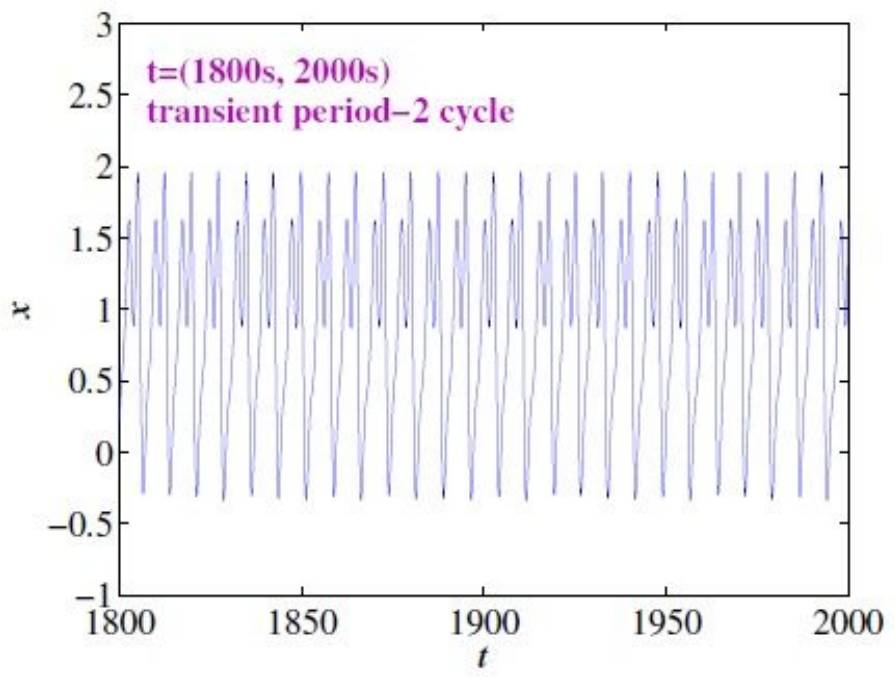

(a)

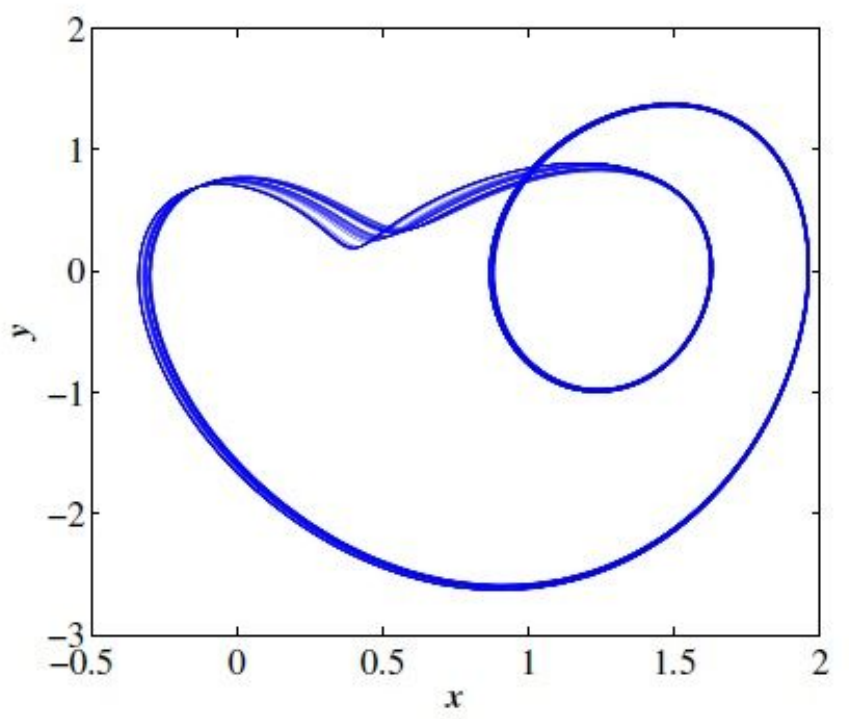

(b)

Figure 14

(a) Time-domain plot of te(1800s, 2200s). (b) Phase portraits of $x$-y axis te(1800s, 2200s).

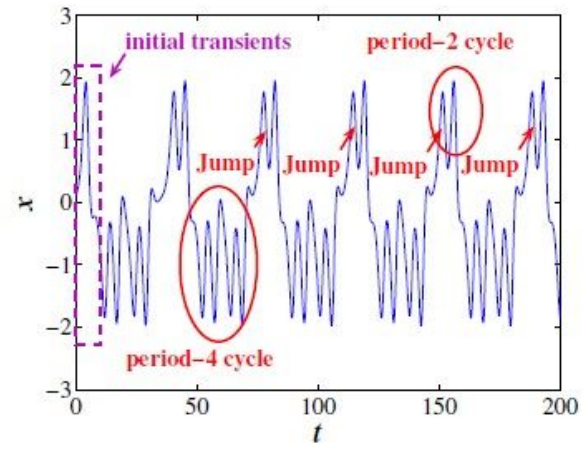

(a)

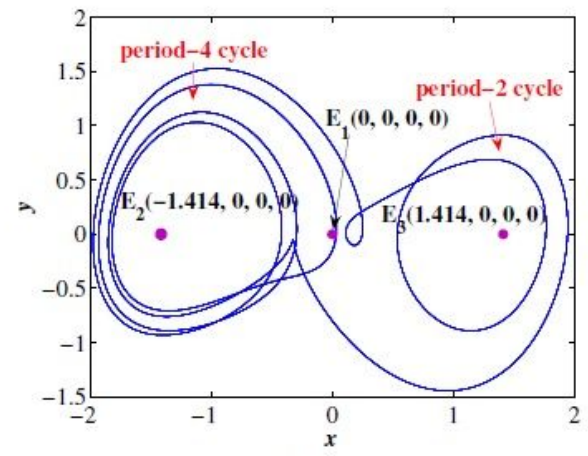

(b)

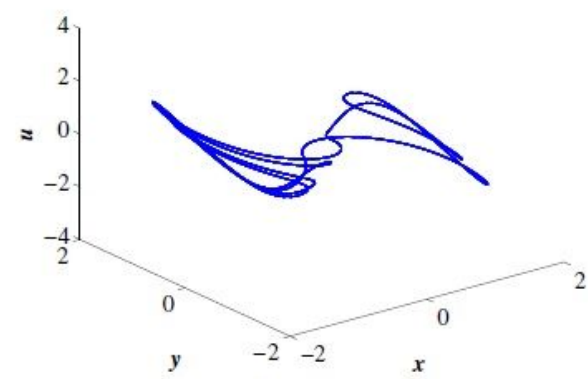

(c)

Figure 15

After neglecting initial transients, when $\mathrm{t2}(20 \mathrm{~s}, 200 \mathrm{~s})$ period-4 cycle jumps to period-2 cycle (a) Timedomain plot. (b) Phase portraits of $x$-y axis. (c) Phase portraits of $x-y-u$ axis

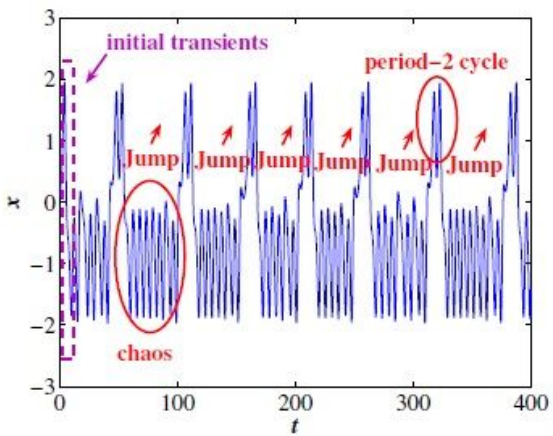

(a)

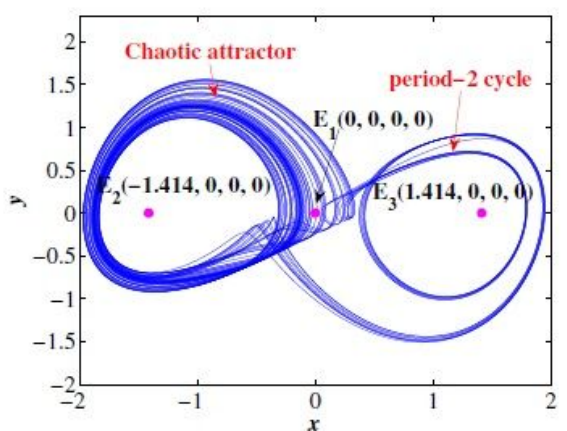

(b)

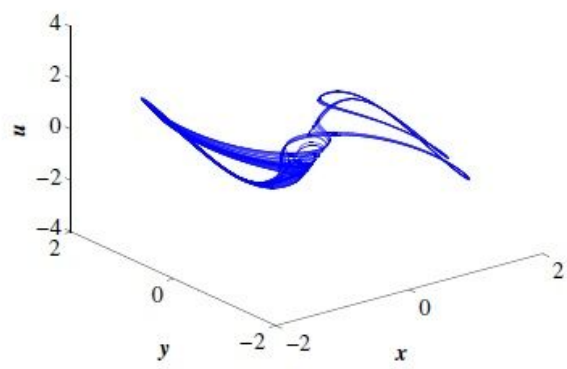

(c) 
Figure 16

After neglecting initial transients, when $\mathrm{t} 2(20 \mathrm{~s}, 400 \mathrm{~s})$ chaos jump to period-2 cycle (a) Time-domain plot. (b) Phase portraits of $x$-y axis. (c) Phase portraits of $x-y$-u axis.

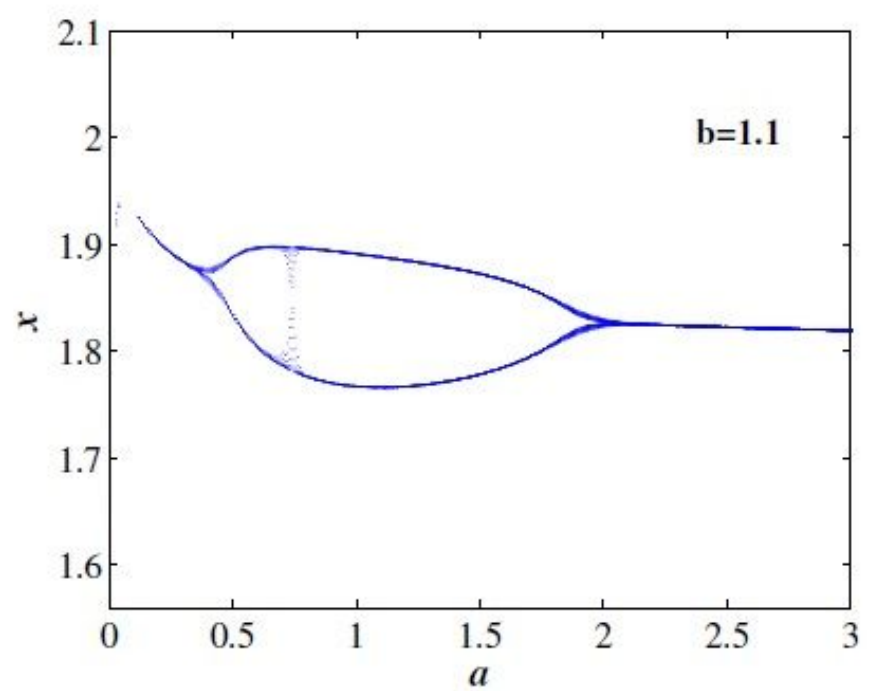

(a)

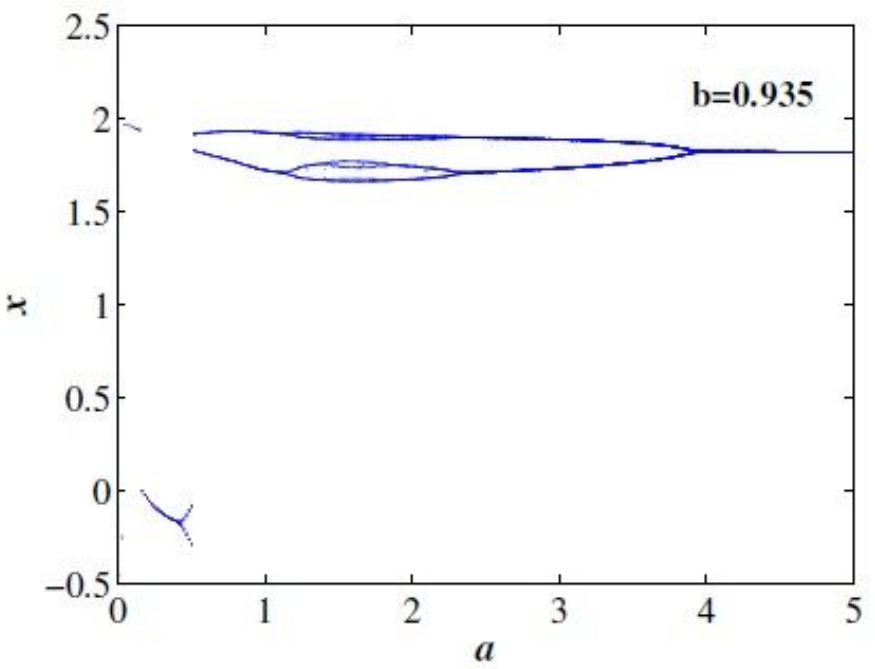

(c)

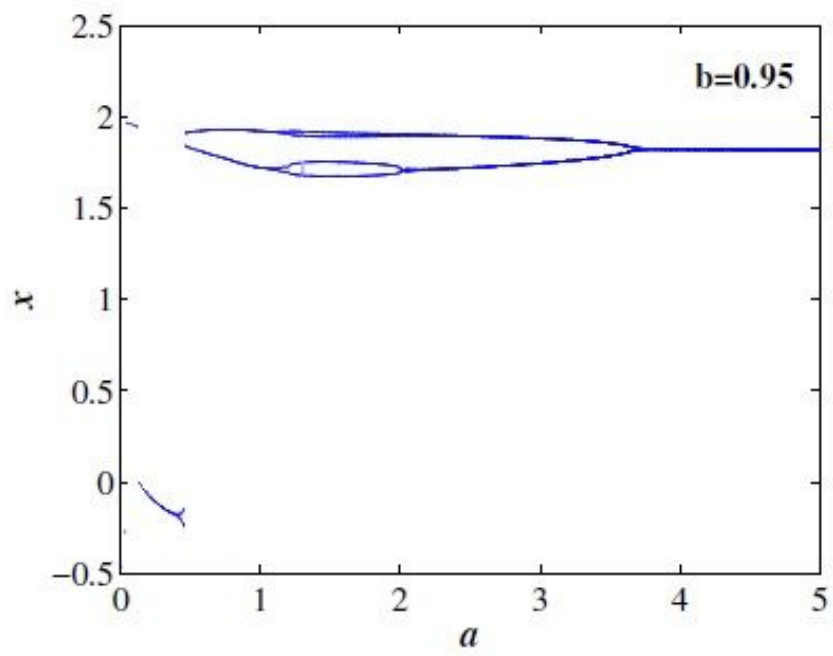

(b)

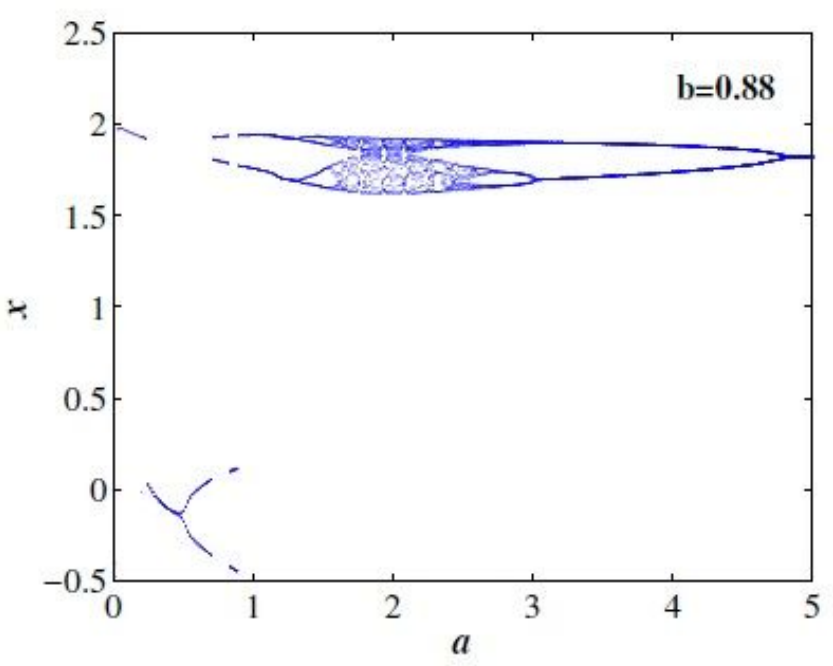

(d)

Figure 17

Bifurcation diagrams of remerging Feigenbaum trees (a) A-period-2 bubble when $b=1.1$. (b) A-period-4 bubble when $b=0.95$. (c) A-period- 6 bubble when $b=0.935$. (d) Feigenbaum tree when $b=0.88$. 


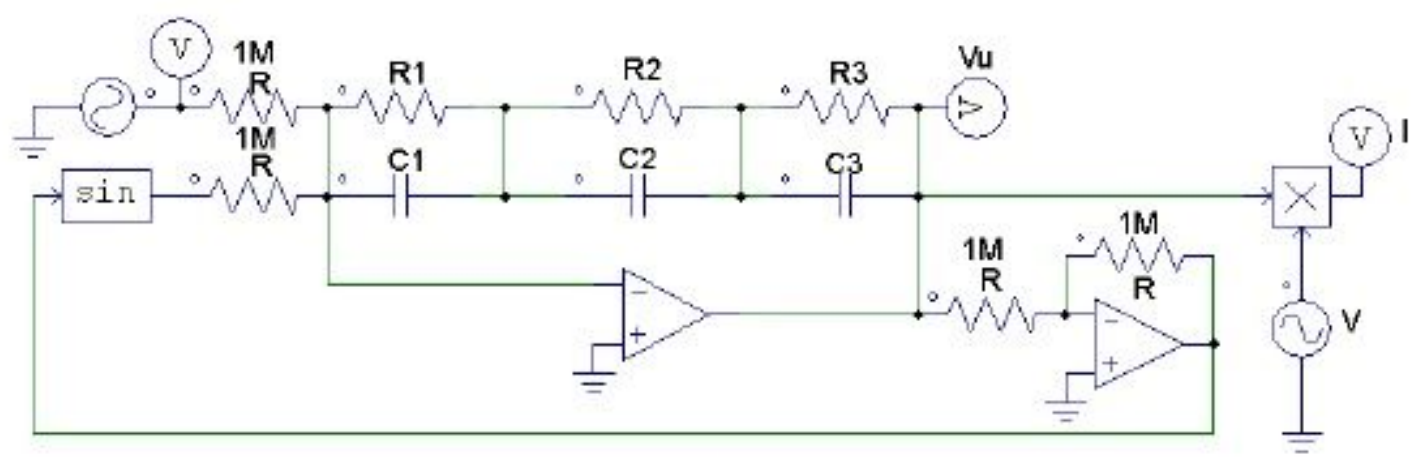

Figure 18

Circuit conguration of the memristor

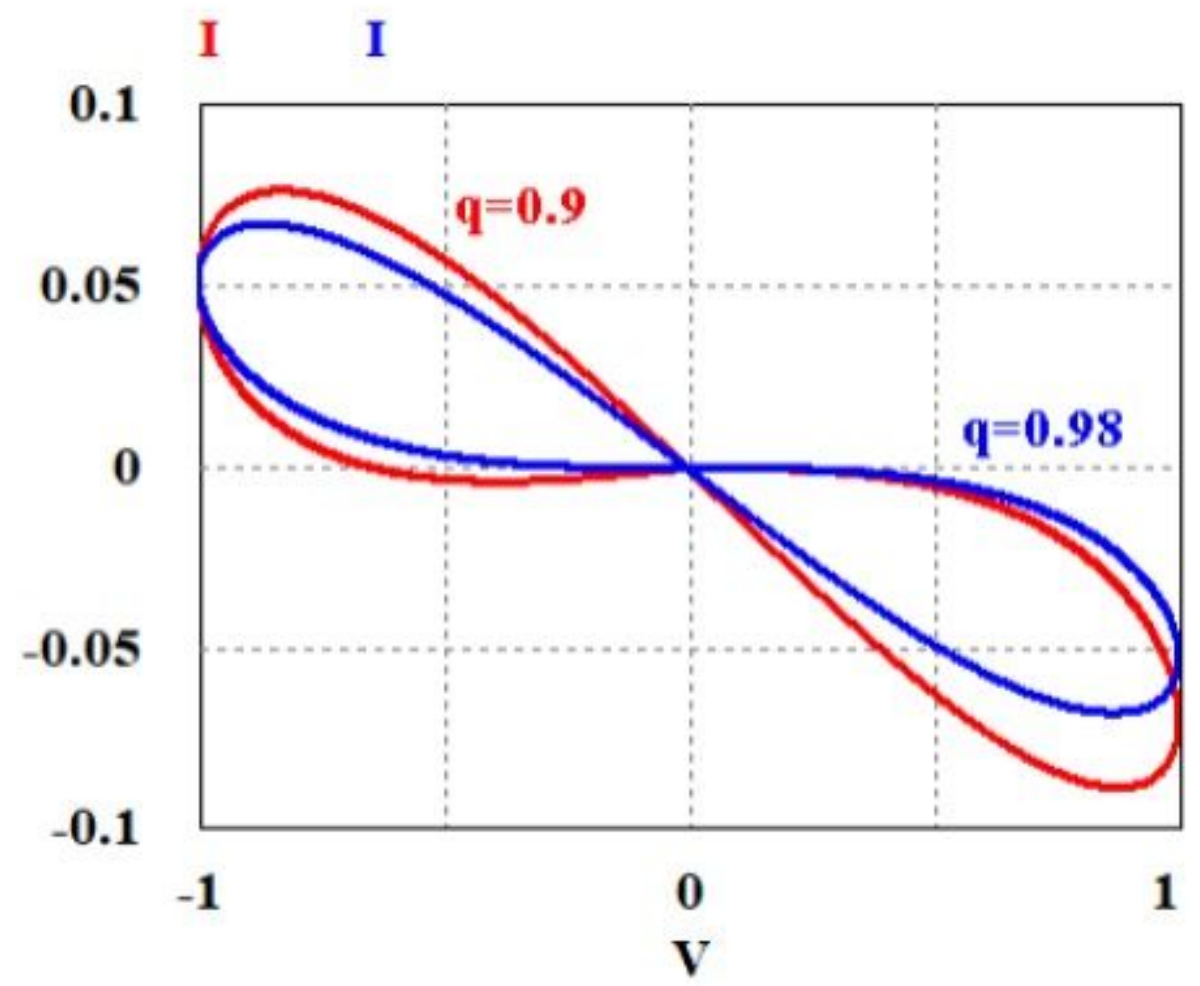

Figure 19

Pinched hysteresis loops obtained from the memris- tor circuit in PSIM simulation when $q=0.98$ (blue curve) and $q=0.9$ (red curve). 


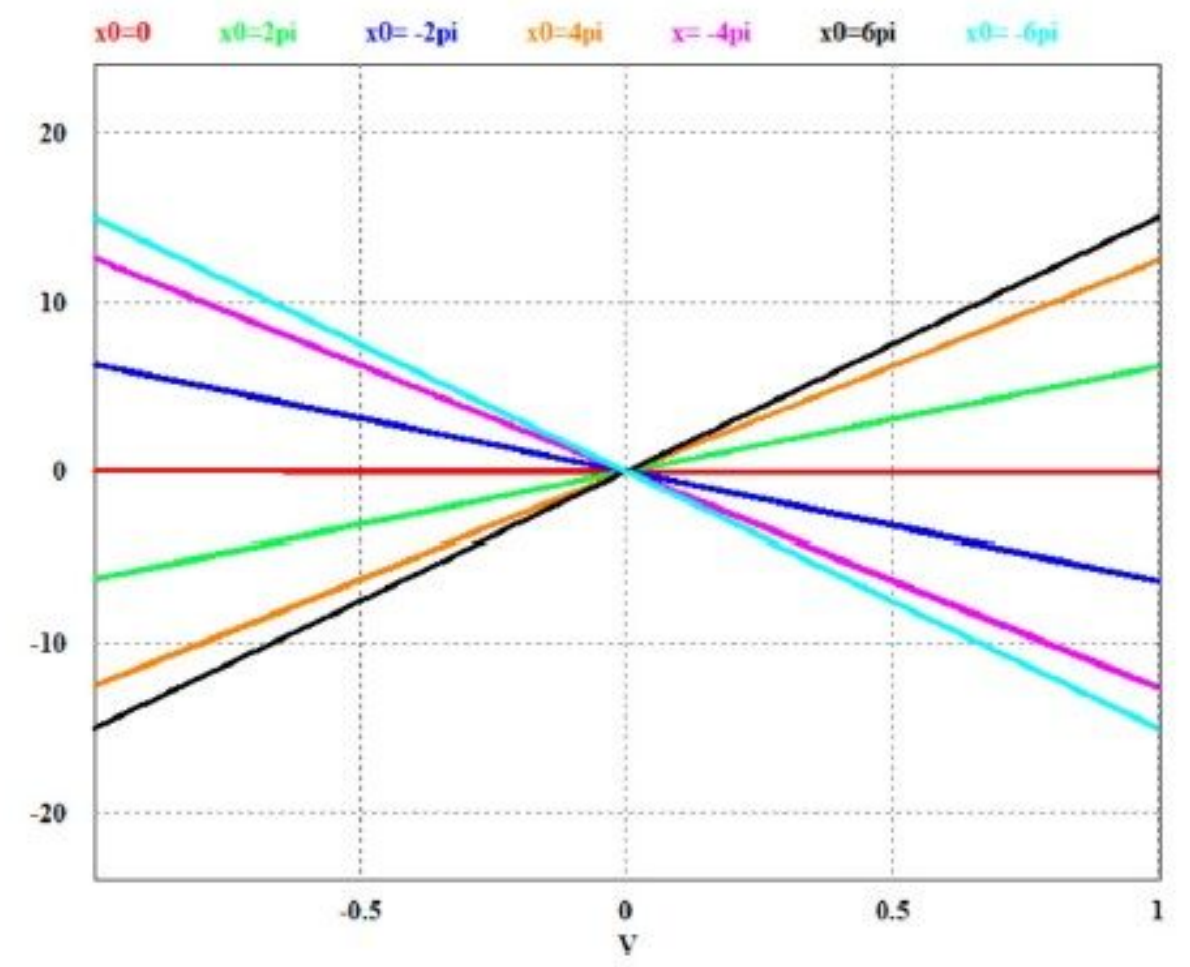

Figure 20

Coexisting pinched hysteresis loops obtained from the memristor circuit in PSIM simulation under different ini- tial capacitor voltages $+6 n,+4 n,+2 n, 0$.

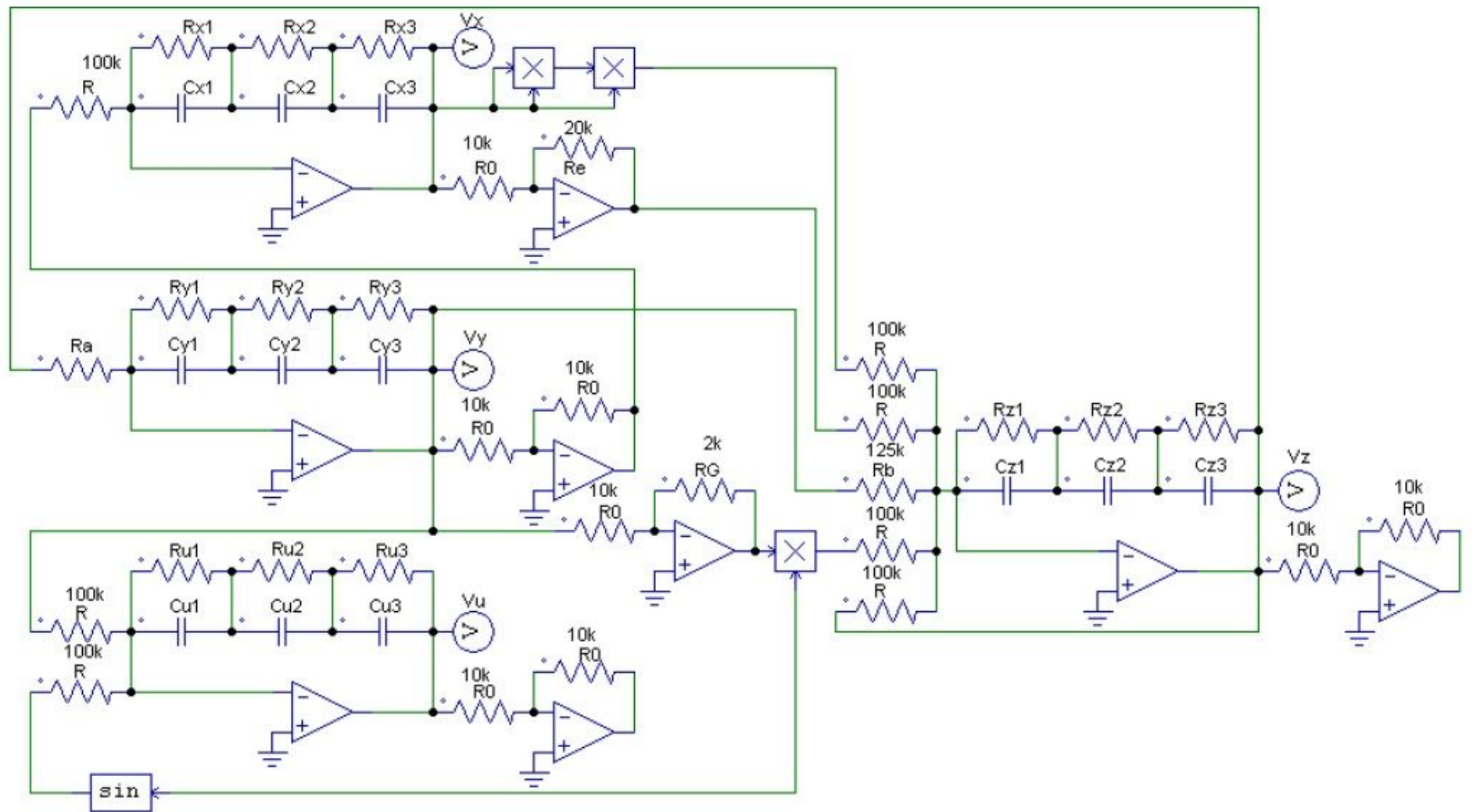

Figure 21 
Circuit conguration of the fractional-order multistable locally active memristive chaotic system

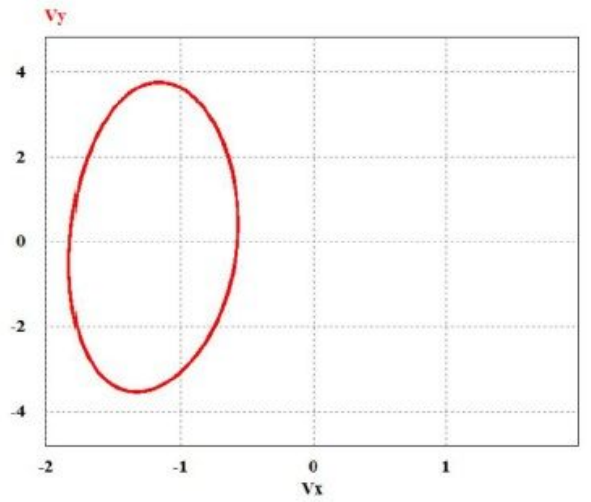

(a)

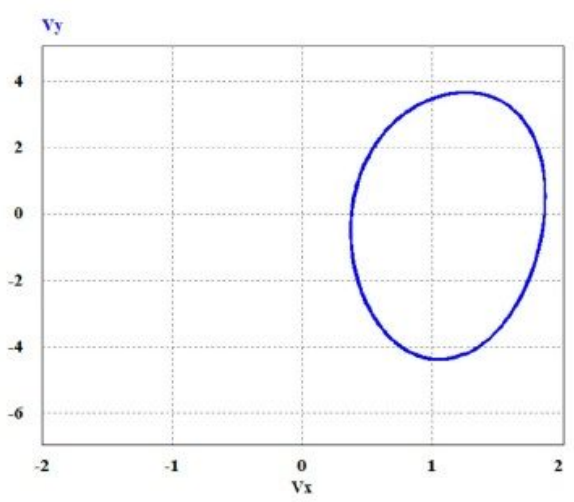

(b)

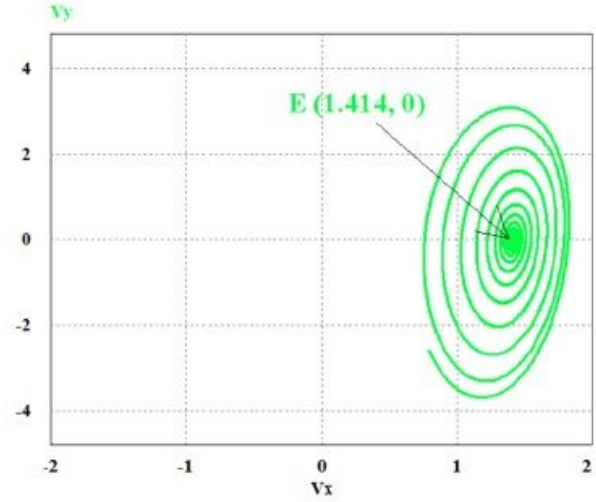

(c)

\section{Figure 22}

Coexisting attractors obtained from the fractional-order chaotic system circuit in PSIM simulation for different initial values and $q=0.9$. (a) Initial values $(0.1,-0.1,-0.1,-0.1)$. (b) Initial values $(0.1,0.1,-0.1,0)$. (c) Initial values $(0.1,0.1,-0.1,-7)$.

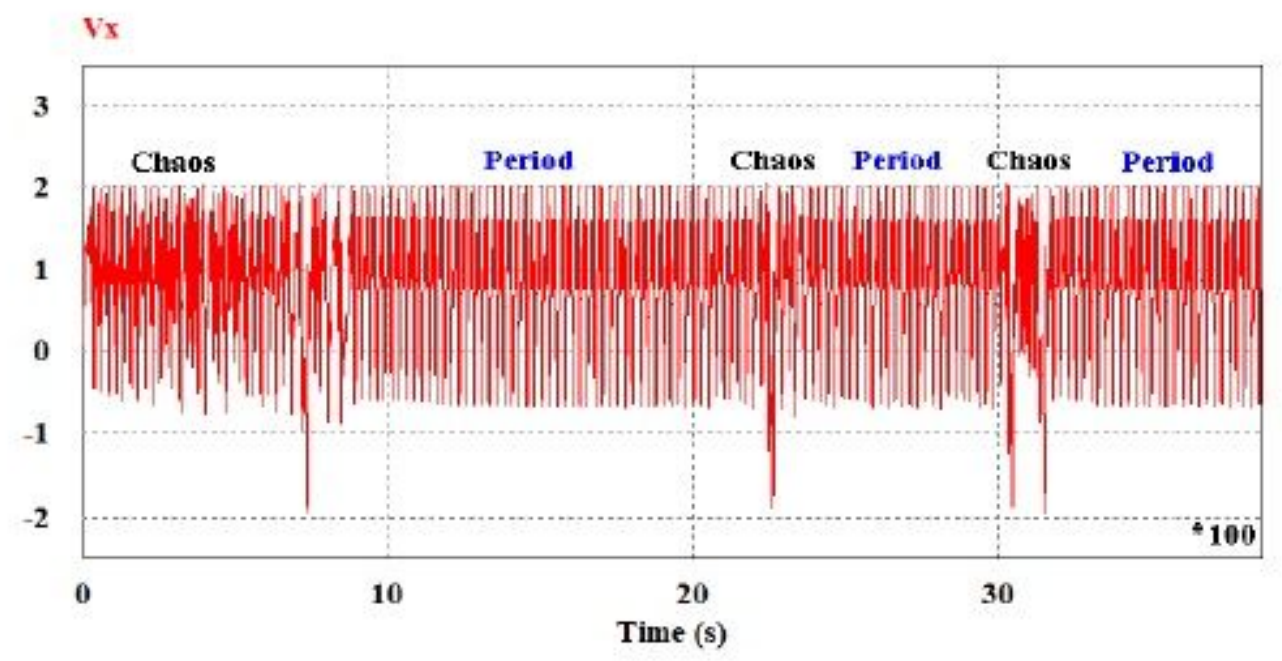

Figure 23

The behavior of transient transition obtained from the fractional-order chaotic system circuit in PSIM simulation 


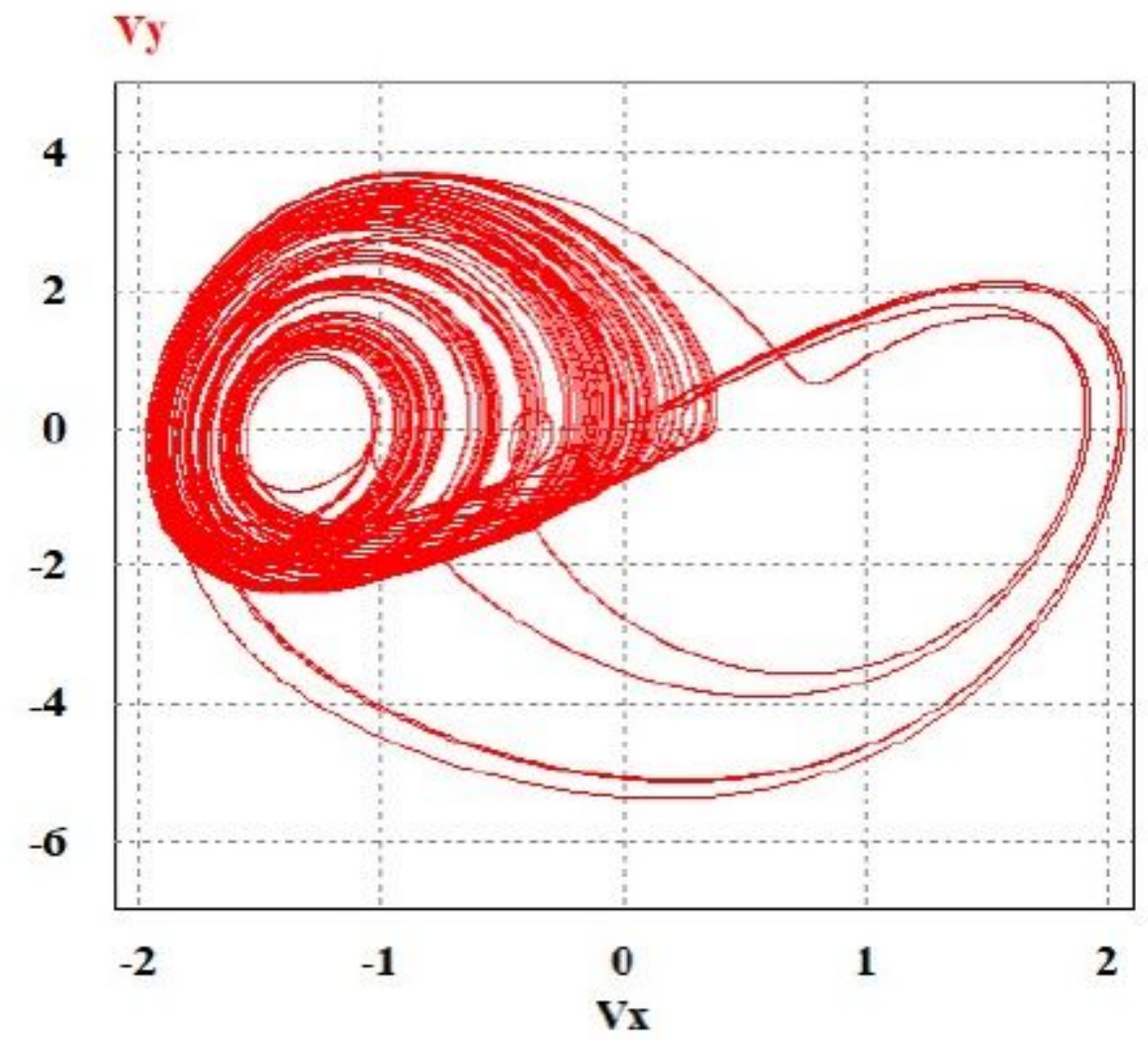

Figure 24

The behavior of state jump obtained from the fractional-order chaotic system circuit in PSIM simulation when $\mathrm{q}=0.97$. 